\title{
Colorectal cancer: Cost-effectiveness of screening and chemoprevention in average risk males
}

Jarrett W. Coffindaffer

West Virginia University

Follow this and additional works at: https://researchrepository.wvu.edu/etd

\section{Recommended Citation}

Coffindaffer, Jarrett W., "Colorectal cancer: Cost-effectiveness of screening and chemoprevention in average risk males" (2006). Graduate Theses, Dissertations, and Problem Reports. 2449.

https://researchrepository.wvu.edu/etd/2449

This Thesis is protected by copyright and/or related rights. It has been brought to you by the The Research Repository @ WVU with permission from the rights-holder(s). You are free to use this Thesis in any way that is permitted by the copyright and related rights legislation that applies to your use. For other uses you must obtain permission from the rights-holder(s) directly, unless additional rights are indicated by a Creative Commons license in the record and/ or on the work itself. This Thesis has been accepted for inclusion in WVU Graduate Theses, Dissertations, and Problem Reports collection by an authorized administrator of The Research Repository @ WVU. For more information, please contact researchrepository@mail.wvu.edu. 


\title{
COLORECTAL CANCER: \\ COST-EFFECTIVENESS OF SCREENING AND CHEMOPREVENTION IN AVERAGE RISK MALES
}

\author{
Jarrett W. Coffindaffer \\ Thesis submitted to the \\ School of Pharmacy \\ at West Virginia University \\ in partial fulfillment of the requirements \\ for the degree of \\ Master of Science \\ in \\ Pharmaceutical Sciences \\ Lesley-Ann Miller, Ph.D., Chair \\ Miklos Auber, M.D. \\ Arthur Jacknowitz, Pharm.D. \\ Virginia (Ginger) Scott, Ph.D.
}

Department of Pharmaceutical Systems \& Policy

\author{
Morgantown, West Virginia \\ 2006
}

Keywords: Colorectal Cancer, Cost-effectiveness, Screening, Fecal Occult Blood Test (FOBT), Flexible Sigmoidoscopy, Colonoscopy, Chemoprevention, Aspirin, Cyclooxygenase-2 Inhibitor (COX-2), Decision Analysis, Markov Model 


\section{ABSTRACT \\ Colorectal Cancer: Cost-Effectiveness of Screening and Chemoprevention in Average Risk Males}

\section{Jarrett Coffindaffer}

This study is an economic evaluation of currently recommended colorectal cancer (CRC) screening procedures, and strategies that incorporate chemopreventive options such as aspirin or a cycooxygenase-2 inhibitor. A decision analysis model was constructed to compare alternative CRC screening strategies. A Markov model was employed to simulate the natural history of CRC. Quality adjusted life years were used as the primary outcome measure. The base case analysis represents the overall cost and effectiveness associated with each screening strategy. Incremental cost-effectiveness ratios (ICERs) were calculated for each screening strategy. One-way sensitivity analyses were performed to assess the factors that have the greatest effect on the cost-effectiveness of screening. The most cost-effective screening strategy was Fecal Occult Blood Test (FOBT); followed by FOBT plus aspirin, colonoscopy, and colonoscopy plus aspirin. The ICER of FOBT was $\$ 13,014.85$ compared to Natural History. The model was sensitive to the costs of FOBT, colonoscopy, and aspirin. 


\section{DEDICATION}

This research is dedicated to

\section{My Grandparents}

Jarrett D. and Phyllis I. Fox

$\&$

Wanda B. Coffindaffer

and the late Wade H. Coffindaffer, Jr.

\section{My Parents}

Wade H. and Linda F. Coffindaffer 


\section{ACKNOWLEDGEMENTS}

I first began working with Dr. Lesley-Ann Miller in the year 2003, after I had completed her decision analysis class at the West Virginia University, School of Pharmacy. It was a project from her class that sparked my interest in colorectal cancer screening. After completing this project, I decided to develop it further into my master's thesis. I appreciate her guidance throughout this time. It has been a long road, but I am excited to see this class project develop into my thesis. This was all possible through her confidence in me.

I would also like to acknowledge the members of my thesis committee: Dr. Virginia (Ginger) Scott, Dr. Arthur Jacknowitz, and Dr. Miklos Auber. I am very grateful to them for both their guidance and support.

To my graduate colleagues and the faculty and staff of Department of Pharmaceutical Systems \& Policy, thank you for always being there. You have always been a cheering section for me. I appreciate all of the support you have given me. These last four years have been a wonderful journey! 


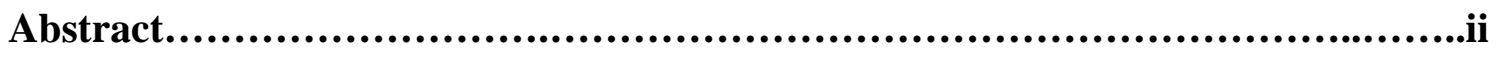

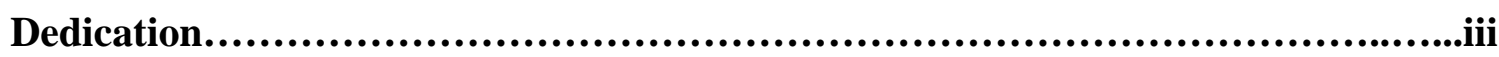

Acknowledgements.....................................................................

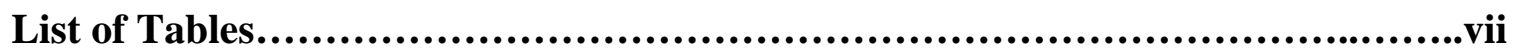

List of Figures........................................................................

1. Introduction........................................................................

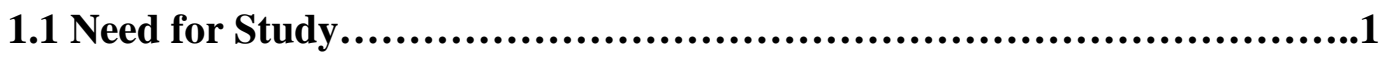

1.2 Specific Aims...............................................................

1.3 Significance of the Study...................................................

2. Background........................................................................

2.1 Colorectal Cancer...........................................................4

2.2 Colorectal Cancer Screening.............................................5

2.3 Treatment of Colorectal Cancer............................................15

2.3.1 Surgery ........................................................15

2.3.2 Radiation......................................................16

2.3.3 Chemotherapy ....................................................17

2.4 Chemoprevention and Colorectal Cancer...................................18

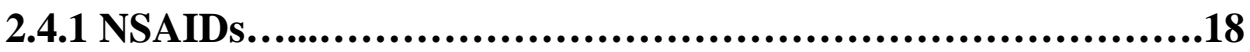

2.4.2 Cox-2 Inhibitor.................................................20

2.5 Cost of Colorectal Cancer...................................................24

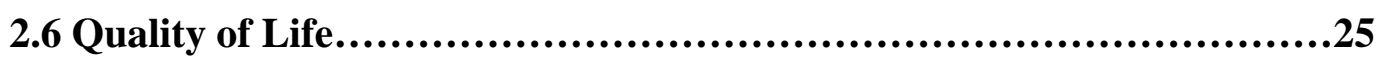

2.7 Cost-Effectiveness....................................................29

2.7.1 Cost-Effectiveness of CRC Screening.............................29 


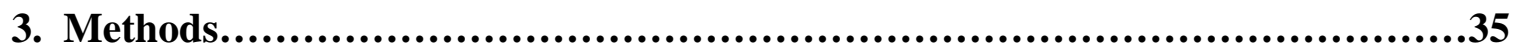

3.1 Model Structure.........................................................35

3.2 Allowable Transitions...................................................41

3.3 Baseline Probabilities and Utilities..........................................43

3.4 Effectiveness and Costs..................................................49

3.5 Outcome Measure....................................................51

3.6 Base-case Cost-effectiveness Analysis.......................................51

3.7 Sensitivity Analyses.....................................................52

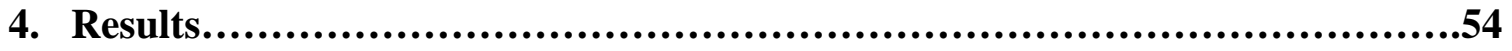

4.1 Base-case Analysis...................................................54

4.2 Sensitivity Analysis...................................................58

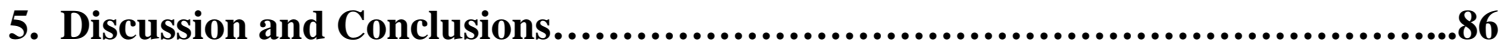

5.1 Review of Findings.....................................................86

5.2 Implications of Study Findings.......................................89

5.3 Study Limitations......................................................89

5.4 Recommendations for Future Research...................................91

5.5 Conclusions............................................................91 


\section{LIST OF TABLES}

Table 1-1...........................................................................6

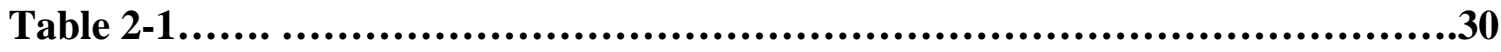

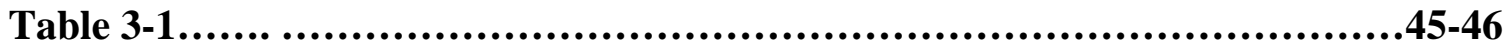

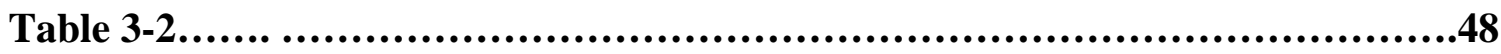

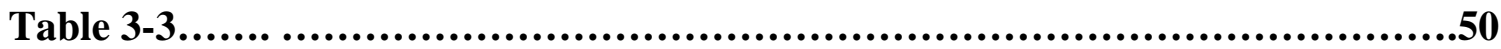

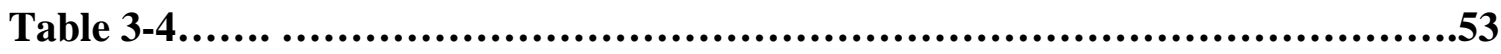

Table 4-1....... .........................................................................56

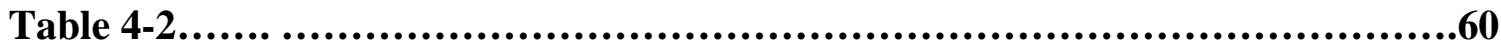

Table 4-3.........................................................................61

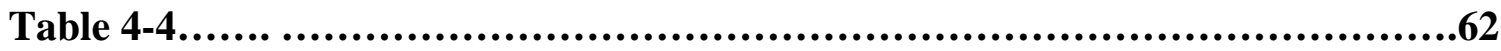

Table 4-5................................................................................63

Table 4-6....... ..................................................................65

Table 4-7.....................................................................66

Table 4-8....... ...................................................................70

Table 4-9.........................................................................71

Table 4-10.....................................................................72

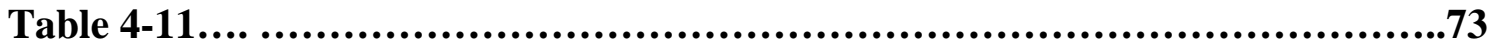

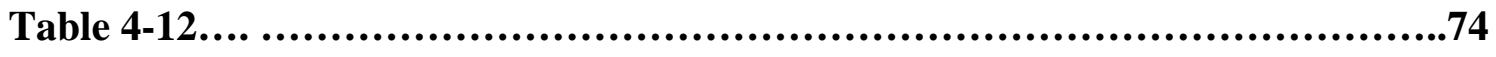

Table 4-13.... .......................................................................75

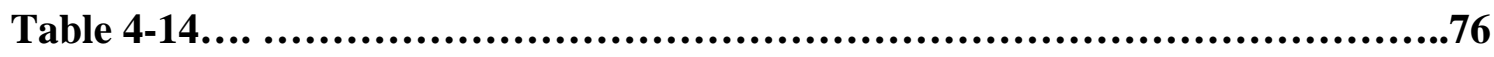

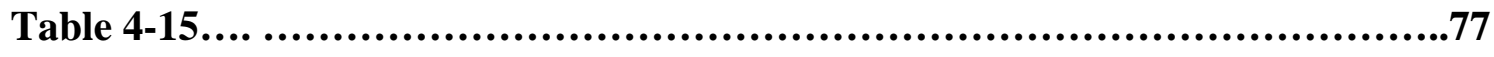

Table 4-16.... ....................................................................78 


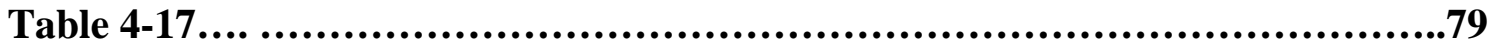

Table 4-18.... .....................................................................80

Table 4-19.... .....................................................................81

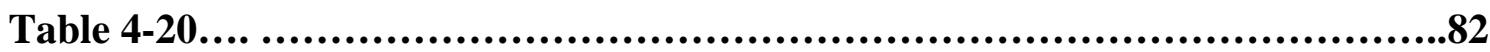

Table 4-21.... ..................................................................83

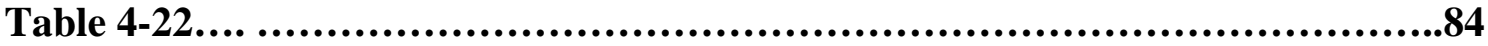

Table 4-23.... ........................................................................85 


\section{LIST OF FIGURES}

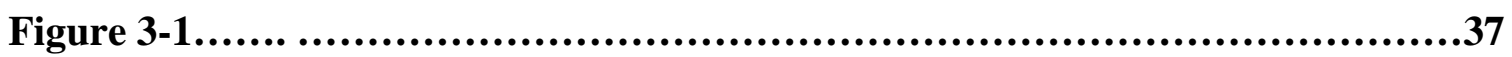

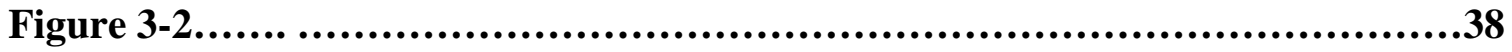

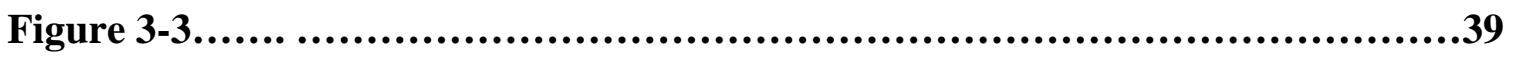

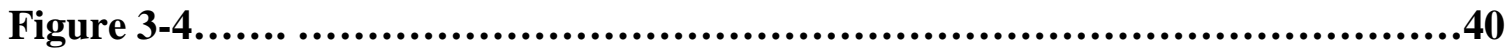

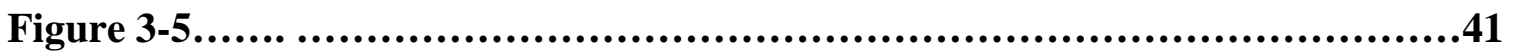

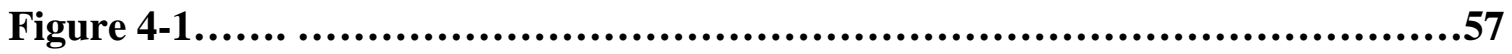




\section{Introduction}

Colorectal cancer (CRC) is the second leading cause of cancer-related deaths in the United States (American Gastroenterological Association (AGA), 2006). The American Cancer Society (ACS) estimates that in 2006, the incidence for colon and rectal cancer cases will be 106,680 and 41,930, respectively, and estimated combined mortality will be 55,170 deaths (ACS, 2006). Screening is used to detect CRC in healthy adults with no signs or symptoms that suggest the presence of CRC or polyps. There are many forms of screening used to detect CRC and recommendations for screening strategies are abundant. The screening modalities for CRC include colonoscopy, flexible sigmoidoscopy (FS), fecal occult blood test (FOBT), and double-contrast barium enema. All are recommended by the ACS, the AGA, and the U.S. Preventive Services Task Force (USPSTF). However, for the detection of CRC, no one screening test or strategy has been recommended as the gold standard by any organization. In fact, each suggests that any form of screening is better than none at all.

\subsection{Need for Study}

In 1996, the USPSTF concluded that there was sufficient evidence to recommend screening for CRC (Bero, Grilli et al., 1998). However, for many years CRC screening has been surrounded by controversy regarding differing guidelines. Consequently, utilization of screening procedures remains low. As a result, there is great concern that the possibility of preventing and controlling this disease is being overlooked. Recommendations for CRC screening vary among professional organizations, and patient and physician opinions regarding the screening procedures are even more diverse. Of all the available CRC screening procedures, no one strategy has been determined to be 
optimal. Therefore, physicians are left to determine which screening procedure is best for their patients. The various recommendations include: FOBT, FS, colonoscopy, or barium contrast enema. While some experts believe FOBT with its annual testing is the most cost-effective method, others believe colonoscopy should be the choice based on its effectiveness alone. Still others recommend other screening methods.

It may be that colonoscopy would be more appealing to patients not at risk due to its 10-year screening interval. Studies are currently being performed to identify the barriers to CRC screening by physicians, patients, and healthcare service providers (Klabunde, Schenck et al., 2006; Klabunde, Vernon et al., 2005; Tabbarah, Nowalk et al., 2005). Identifying the most cost-effective screening strategy will help in optimizing resources for screening utilization. The AGA recommends that routine screening for CRC be widely adopted. However, according to a case-control study by Selby and colleagues (Selby, Friedman et al., 1992), screening rates for CRC have yet to increase as compared to screening for breast or cervical cancer in women. Despite the modest cost of the initial screening tests, CRC is perceived as having potential costly follow-up procedures for diagnosis and surveillance.

In the current literature, there have been no published studies utilizing a decision analysis model comparing the screening procedures of FOBT, FS, and colonoscopy with each other while also measuring the effectiveness of chemoprevention. All three screening procedures examined in this study are currently recommended by professional organizations, such as the ACS and AGS, but there is no consensus in the literature as to which procedure is most cost-effective. This study will evaluate the cost-effectiveness of FOBT, FOBT plus FS, and colonoscopy, in addition to evaluating the combination of 
chemopreventive options, such as aspirin and cyclooxygenase-2 inhibitor (COX-2), with each strategy.

\subsection{Specific Aims}

The purpose of this study is to conduct an economic evaluation of currently recommended CRC screening procedures, and strategies that incorporate chemopreventive options such as aspirin or a cycooxygenase-2 inhibitor (Cox-2). The objectives of this study are: 1) to determine the cost-effectiveness of three of the currently recommended CRC screening procedures alone, and also in combination with two chemopreventive agents (nine strategies in all); 2) to determine which of the nine strategies yields the most savings in cost, greatest increase in survival, and in qualityadjusted survival (quality adjusted life years, QALYs).

\subsection{Significance}

The study results will be a valuable addition to the scientific literature in the field of CRC screening. In addition to its academic significance, the study results have important implications for decision makers of CRC screening; physicians, administrators, and patients. Most importantly, the results provide an economic evaluation of the nine screening strategies modeled in this study. These nine strategies have not been modeled together in the current literature. 


\section{Background}

\subsection{Epidemiology of Colorectal Cancer}

Colorectal cancer (CRC) is the second leading cause of cancer-related deaths in the United States (American Gastroenterological Association (AGA), 2006). It is also the third most common type of cancer among American men and women. In 2001, it was estimated that 135,400 new cases of colorectal cancer developed and that 56,700 individuals with CRC died (American Cancer Society (ACS), 2001). Colorectal cancer incidence is increasing each year while new forms of screening, prevention and treatment are sought. The ACS estimates that in 2006, the incidence for CRC will be 148,61, and estimated mortality will be 55,170 deaths from CRC (ACS, 2006). Ninety-three percent of CRC cases occur in people age 50 or older and $80 \%$ of deaths occur in people over the age of 65 (ACS, 2006). The risk of developing CRC increases with age and every person has a one in twenty chance of developing CRC in their lifetime. Although the cause of CRC is not known, risk factors associated with developing CRC are family history, age (50 years and older), ethnicity, and/or lifestyle and dietary choices.

Approximately 10 percent of all colon cancers are hereditary, that is, directly caused by specific inherited genetic abnormalities (Cancer Research and Prevention Foundation (CRPF), 2004). While sporadic, non-hereditary cancers usually appear after age 50, hereditary colon cancers often occur in younger people. When cancer is prevalent in a family, but no specific genetic change has been linked to the cancers, the family is said to have familial CRC.

With regard to race, blacks have a $40 \%$ greater chance of developing CRC than whites (Centers for Disease Control and Prevention (CDC), 2002). The five year survival 
rate of CRC is higher for whites than blacks with localized cancer (90\% versus $83 \%$, respectively) and regionally spreading cancer (65\% versus 53\%) (Surveillance, Epidemiology, and End Results (SEER), 2003). These survival differences exist even when other factors, such as age, gender, and geography, are considered. Over the last decade there has been a decrease in death due to CRC (SEER, 2003). The death rate for white males and females dropped between 1990 and 1998, 2.2\% and 1.8\% respectively. Death rates have also dropped for blacks in that same time period, although approximately 50\% of that for whites (CDC, Morbidity and Mortality Weekly Report, 2002). This drop in CRC deaths may be attributable to a shift in emphasizing cancer prevention by many national programs. March is National CRC Awareness month, and the symbol of a blue star was recently introduced by the National Colorectal Cancer Roundtable (NCCRT) and will now represent the fight against colorectal cancer just as the pink ribbon signifies breast cancer and the red ribbon depicts HIV/AIDS. Also, the CDC's CRC Prevention and Control screening campaign informs men and women aged 50 years or older about the importance of having regular CRC screening tests. Many public and private insurers are beginning to cover CRC screening. In the state of West Virginia, the Public Employees Insurance Program (PEIA) provides 100 percent coverage for CRC screening for all employees over the age of 50.

\section{Detection of CRC}

Colorectal cancer typically grows slowly over a period of several years. The precancerous lesions are small benign growths called polyps. Early detection and removal of polyps (polypectomy) is the optimal method of preventing the development of 
CRC (AGA, 2002). Although many cases are asymptomatic, the identification of symptoms can aid in the early detection of CRC. Symptoms may include: blood in stool, change in bowel habits, abdominal pain, continuous feeling the need for bowel movement, and fatigue. Because of the non-specific nature of many of these symptoms, they are often ignored, especially at the onset of the disease.

Malignancy usually starts in the innermost layer of the sigmoid colon and moves outwardly as it progresses. As the tumor grows through the layers of tissue, the extent of penetration determines the stage of the disease. Identifying the stage of CRC helps to determine treatment strategies and provides an estimate of the survival rate for the patient. Also, the stage of disease is the strongest determinant of prognosis with the fiveyear survival of patients in stage four (the most advanced stage) estimated to be less than ten percent. Table 1 identifies and describes the various stages of colorectal cancer.

\section{TABLE 1-1. Stages of Colorectal Cancer}

\begin{tabular}{|l|l|}
\hline Stage 0 & $\begin{array}{l}\text { The cancer is in the earliest stage and has not grown beyond the inner layer } \\
\text { of the colon. }\end{array}$ \\
\hline Stage 1 & $\begin{array}{l}\text { The cancer has grown through the inner layer of the colon but has not spread } \\
\text { through the colon wall. }\end{array}$ \\
\hline Stage 2 & $\begin{array}{l}\text { The cancer has grown through the wall of the colon but has not spread to } \\
\text { nearby lymph nodes. }\end{array}$ \\
\hline Stage 3 & The cancer has spread to nearby lymph nodes, not to other parts of the body. \\
\hline Stage 4 & The cancer has spread to distant sites, such as other organs. \\
\hline Recurrent & $\begin{array}{l}\text { The cancer has returned after treatment. It may recur in the colon or other } \\
\text { parts of the body. }\end{array}$ \\
\hline
\end{tabular}




\subsection{Colorectal Cancer Screening}

Screening is used to detect CRC in healthy adults with no signs or symptoms that suggest the presence of CRC or polyps. Individuals who present with signs or symptoms of CRC should be offered appropriate diagnostic evaluation. Choice of screening strategies should be based on both patient preference and on an individual patient's level of risk, which takes into account their personal and family medical history.

Currently there are many forms of screening used to detect CRC and recommendations for screening strategies are abundant. The screening modalities for CRC include FOBT, FS, colonoscopy, and double-contrast barium enema (DCBE). All are recommended by the ACS, the AGA, and the U.S. Preventive Services Task Force (USPSTF). However, for the detection of CRC, no one screening test or strategy has been recommended as the gold standard by any organization. It is recommended that at least one screening strategy be employed with the patient and physician determining the most appropriate method.

In 1996, the USPSTF concluded that there was sufficient evidence to recommend screening for CRC (Bero, Grilli et al., 1998). However, for many years CRC screening has been surrounded by controversy regarding which of the various guidelines to utilize. As a result of this ambiguity, and the low compliance rates of screening, many physicians tend to give priority to other preventive services. For patients, health policy factors such as access to care, costs, and reimbursement have been shown to influence compliance with CRC screening (Batelle Corp., 1998). The National Colorectal Cancer Roundtable (NCCRT) has found that the majority of adults in the U.S. are not currently being screened for CRC (NCCRT, 2005). This limits the opportunity to reduce CRC-related 
mortality by $50 \%$ or more as envisioned by the Colorectal Cancer Review Group (National Cancer Institute (NCI), 2000). A report by the NCCRT states that to increase CRC screening rates, the issues of patient and physician barriers to screening, lack of universal insurance coverage, lack of incentives to motivate adherence, and expanded infrastructure must be addressed (Levin, Smith et al., 2002). Efforts are already underway to promote the importance of colorectal cancer screening and increase its use. For example, the CDC has implemented a program called "Screening for Life" to inform men and women aged 50 years or older about the importance of having regular CRC screenings. Partners in this initiative include the Center for Medicare \& Medicaid Services (CMS), the NCI, and local health departments.

Although studies have provided evidence that screening is effective in reducing mortality from CRC as mentioned previously, utilization of screening services is low. In a study by the CDC using 1999 Behavioral Risk Factor Surveillance System (BRFSS) data, only $44 \%$ of respondents (male and female, $>49$ years of age) reported undergoing any form of CRC screening (colonoscopy, FS, or FOBT) within the recommended period of time (Sandler, Halabi et al., 2003). The survival rate of CRC is much higher when the cancer is detected early. For white males, the five-year survival rate for cancer detected in the early, localized state is $90 \%$, as compared to detection in a regionally spreading state which has a five-year survival rate of $65 \%$.

Studies have been conducted to determine possible barriers to CRC screening for both patients and physicians. Woolf and colleagues (Woolf, et al, 2000) found that the primary barrier for patients is the negative opinion they have regarding the screening procedures. Many patients associate pain or discomfort with CRC screening, making 
them less likely to discuss this concern with their physician. Many physicians, though they may know the importance of CRC screening, still do not recommend screening (USPSTF, 1996). Some physicians are aware of the most recent guidelines for CRC screenings, but may not be able to accurately assess the risk associated with individual patients. They may be limited by restrictions on patients' insurance, or they may not be impressed by the data that supports the benefit of CRC screening (USPSTF, 1996). Some healthcare professionals report failure to recommend a FOBT due to forgetting it, thinking that the test is ineffective, or not wanting to inconvenience patients (Vernon, 1997; Peterson and Vernon, 2000). When physicians report reasons for not offering sigmoidoscopy, they cite discomfort for the patient, lack of time, little probability of detecting a significant lesion, their own inconvenience, insufficient training or experience in performing the procedure and cost (Batelle Corp., 1998; Cooper, Fortinsky et al., 1998).

\section{Fecal Occult Blood Test}

The FOBT, also known as the stool blood test, is a non-invasive method of CRC screening recommended to be performed yearly. This test detects traces of blood in the stool, which can be a sign of CRC. The test consists of individuals obtaining samples of stool using an at-home kit. The samples are then sent to a lab for testing. Sensitivity of a single FOBT is low, around $30-50 \%$, but repeated annual testing can detect as many as 92\% of colorectal cancers (Reeves, Newcomb et al., 1996). Many trials have shown that annual FOBT screenings can reduce the mortality of CRC in the range of 15 to $33 \%$ (Kune, 2000; Arguedas, 2001; Ladabaum, 2001; Lieberman, 2001; Autier, 2003; Daniels, 
1995). One concern is that false positive FOBT test results can lead to unnecessary diagnostic exams, usually colonoscopy, which increases cost and may cause discomfort for patients.

A newer type of stool blood test is the fecal immunochemical test (FIT, or iFOBT). This test is performed differently than the FOBT. With iFOBT, a special brush is used to capture a water sample from within the toilet bowl - it is not necessary to handle the stool. Unlike FOBT, iFOBT will not react with non-human hemoglobin (a component of red blood cells), vitamins, medications, or peroxidase found in some foods that can lead to false positive test results. This test may be more specific for detecting loss of blood from the lower part of the intestine because this blood is less degraded during transit. In April 2002, the ACS Colorectal Cancer Advisory Group concluded that the iFOBT has some advantages that merit revision of their guideline statement for FOBT, to include the iFOBT. Based on the advisory group's report, the ACS's Recommendations for Screening and Surveillance for the Early Detection of Adenomatous Polyps and Colorectal Cancer now include the statement, "in comparison with guaiac-based tests for the detection of occult blood, immunochemical tests are more patient-friendly, and are likely to be equal or better in sensitivity and specificity."

\section{Flexible Sigmoidoscopy}

Flexible sigmoidoscopy (FS) is a procedure in which a thin, flexible, lighted tube with a tiny video camera on the end is inserted into the rectum and lower third of the colon. The scope allows inspection of the rectum and the sigmoid colon. This allows the physician to examine the inside of the rectum and the lower colon to find and remove any 
polyps that have developed. For screening purposes, FS is recommended every five years by the ACS and the AGA. The interval is shorter than that of colonoscopy because FS is a less sensitive test. Screening with FS appears to detect about 70\% of all advanced neoplasia (stages three and four) in the colon, and the detection rate is upwards of $75 \%$ if FS is combined with FOBT (Lieberman, Weiss et al., 2001).

\section{Fecal Occult Blood Test plus Flexible Sigmoidoscopy}

In addition to the ACS, the AGA and the USPSTF also recommend that FOBT and FS be performed together for CRC screening (Winawer, Fletcher et al., 2003; USPSTF, 2002). The USPSTF suggests that when both tests are used, FOBT should be performed first. A positive result is an indication for colonoscopy, obviating the need for the FS. If either FOBT or FS yields abnormal results, complete colonoscopy should be performed.

\section{Colonoscopy}

Colonoscopy is the most specific and most sensitive of all the screening strategies. Consequently, this screening method is able to detect more cases of CRC or large polyps which can then be removed either by an attachment to the colonoscope or surgically (DuBois, Giardiello et al., 1996). Colonoscopy, like FS, is a procedure in which a thin, flexible, lighted tube with a tiny video camera on the end is inserted into the rectum and colon. This allows the physician to examine the inside of the rectum and entire colon to find and remove any polyps that have developed. Before a colonoscopy is performed, a patient must take a hypersomotic laxative such as magnesium citrate or 
dibasic sodium phosphate. These solutions are used to empty and prepare the colon for the procedure. However, colonoscopy involves greater inconvenience, risk, and cost to patients than the other screening tests available. Risks of colonoscopy include discomfort during the procedure and possibly after the procedure, gastrointestinal bleeding, perforation of the colon, and death, although the risk of death is quite low. It is estimated that the risk of death from perforation-related complications from colonoscopy is about $0.11 \%$ with older persons and those exhibiting symptoms are also at greater risk (Ransohoff, Land et al., 1991).

Colonoscopy is the most expensive procedure and can cost between $\$ 975$ and \$1500 (Wagner, et al., 1996; Frazier, et al., 2000; Khandker, et al., 2000). This is significant when compared with FOBT (\$35-\$55), FS (\$250-\$400), and Double Contrast Barium Enema (DCBE) (\$250-\$500) (Pignone, Saha et al., 2002). The recommendation by the ACS and AGA is for colonoscopy to be performed on an average-risk patient every ten years beginning at the age of 50, otherwise every 1-2 years for an individual at high-risk. High risk individuals are those who have previously had CRC, or have had a close blood relative, such as a parent or sibling, who has had CRC. According to Lieberman (Lieberman, Holub et al., 2005), asymptomatic screening accounts for almost a third of colonoscopies performed in persons age 50 year and older. As of July 1, 2001, United States Federal law entitled Medicare beneficiaries to a colonoscopy every ten years, however the proportion of physicians recommending this screening to these individuals remains low (CMS, 2001; Peleg, Maibach et al., 1996). Additionally, Lieberman (Lieberman, Holub et al., 2005) found that colonoscopy utilization varies based on age, gender, and race. 
Colonoscopic screening, although effective, may not be the most desirable screening method for everyone. Data from a number of recent polyp prevention trials suggests that colonoscopy with polypectomy may not lead to a large reduction in CRC cases or mortality (Pabby, 2005; Robertson, Greenbert et al., 2005). Also, screening colonoscopy has a rate of serious complications that may be as much as ten times the rate for FS screening (Nelson, McQuaid et al., 2002). Thus, screening colonoscopy must be weighed against the less invasive screening tests to determine the incremental benefits.

\section{Double-Contrast Barium Enema}

Double-contrast barium enema (DCBE) is another form of screening, which is also recommended by the ACS. It is suggests that DCBE be performed once every 5-10 years. This procedure involves an X-ray examination of the colon and rectum. The colon is filled with a liquid contrast material containing barium. The barium is not excreted, which allows the outline of the colon to appear on the X-ray film. In a casecontrol study by Mandel and colleagues (Mandel, Bond et al., 1993), screening with DCBE was associated with a $33 \%$ reduction in CRC deaths. The cost of the DCBE is lower than that of the colonoscopy, but higher than FOBT and FS. Patient concerns associated with DCBE include the feeling of discomfort during the procedure. Also, there is a very small risk of perforation $(1 / 25,000)$ which may occur due to the pressure exerted on the colon wall during the procedure (Glick, Ralls et al., 2000). However, the effectiveness of barium enema is unclear since documentation is minimal and usage has declined. The current study did not include DCBE as a screening strategy due to uncertainty regarding its effectiveness. 


\section{The Future of CRC Screening}

There are emerging technologies which hold substantial promise in CRC screening. These include virtual colonoscopy (Mulhall,Veerappan et al., 2005), magnetic resonance (MR) colonography and fecal DNA tests (Osborn and Ahlquist, 2005). Virtual colonoscopy (or CT colonography) is non-invasive and does not cause major complications. It is an x-ray technique using high-speed CT scanning and complex software to obtain a two dimensional image of the colon. It differs from colonoscopy in that it does not involve the direct visualization of the surface of the colon using highresolution video equipment. As with colonoscopy, this procedure requires the same type of bowel preparation and may still be uncomfortable for the patient. With colonoscopy, the discomfort arises from the insertion of the colonoscope and air insufflation. However, the discomfort of virtual colonoscopy is from air insufflation only, making the procedure somewhat less uncomfortable.

A study by Pineau and colleagues (Pineau, Paskett et al., 2003) determined that virtual colonoscopy has a high sensitivity and specificity of detecting significant colorectal lesions. The sensitivity and specificity for an precancerous polyp was determined to be $93.8 \%$ and $96.0 \%$, respectively in a study by Prickhardt and colleagues (Prickhardt, Choi et al., 2003). In a 1999 cost-effectiveness study, it was found that screening with virtual colonoscopy costs $\$ 24,586$ per life-year saved, compared with \$20,930 per life-year saved by colonoscopy screening (Sonnenberg, Delco et al., 1999). Furthermore, the authors concluded that screening by colonoscopy remained more cost- 
effective even if the sensitivity and specificity of virtual colonoscopy both rise to $100 \%$. For this reason, virtual colonoscopy was not used as a comparator in this study.

However, patients who choose not to have a standard colonoscopy, whether due to the invasiveness of the procedure, or the slightly increased risk of bowel perforation, may then choose to have a virtual colonoscopy. However, a significant disadvantage of virtual colonoscopy is that a subsequent colonoscopy must be performed if polyps are

discovered during the procedure and are to be removed. Although technology is continually improving, some of the new screening procedures, such as genetic (DNA) testing of stool samples, are not ready for widespread use.

\subsection{Treatment of Colorectal Cancer}

There are three main approaches to the treatment of colorectal cancer; surgery, radiation therapy, and chemotherapy. In addition, targeted therapies called monoclonal antibodies are now being administered as well. Two or more types of treatment may be used at the same time or one treatment may follow the other treatment, depending on the stage of the diagnosed cancer.

\subsubsection{Surgery}

Colorectal cancer treatment is based mainly on the size, location, and extent of the lesion. The primary treatment for CRC is surgery, which almost always involves the removal of part of the rectum or colon where the cancerous lesion is found. This procedure is called a partial colectomy. The degree to which the cancer has spread determines the amount of colon removed. The healthy parts of the colon or rectum are 
then reattached. Also, a colostomy may be performed if, after the diseased portions of the colon, rectum and tissue are removed, the healthy tissues cannot be sewn back together. In this procedure, part of the colon is brought through an incision in the abdominal wall and formed into an artificial opening (stoma) to allow the discharge of feces into a lightweight bag attached to the skin. A colostomy may be temporary or permanent. A temporary colostomy is sometimes needed to allow the lower colon or the rectum to heal after surgery. Later, in a second procedure, the surgeon will reconnect the healthy sections of the colon or rectum.

\subsubsection{Radiation}

Radiation, used to destroy cancer cells and/or shrink tumors, can be used alone or in addition to surgery. Typically, radiation is used in stages two, three and four of CRC. This is to ensure all cancer cells are suppressed or destroyed. Unfortunately, this is not always successful in later stages of disease (stages three and four). Radiation therapy may be used before surgery to shrink a tumor so that it is easier to remove, or may be utilized following surgery to decrease the chances of cancer occurrence. There are two methods used to expose the colon/rectum to radiation: 1) using a machine outside the body, referred to as external radiation therapy; or 2) administering radioactive materials through thin plastic tubes into the intestinal area, called internal radiation therapy. Radiation therapy is usually given on an outpatient basis for approximately five to six weeks (five days a week). Generally, radiation has fewer side effects for the patient than the alternative, chemotherapy. 


\subsubsection{Chemotherapy}

Chemotherapy is used to destroy cancer cells. Given orally or via the bloodstream these drugs enter the bloodstream and travel through the body to kill cancer cells outside of the colon. Chemotherapy may be given after surgery to kill any cancer cells that remain or as a preventive measure after surgery to assure the cancer does not spread. This preventive measure is called adjuvant therapy. Like radiation therapy, chemotherapy is usually utilized in the second, third and fourth stages of CRC, but is not always successful during the latter two stages of CRC. Chemotherapy is most often given in cycles - a treatment period followed by a rest period to allow the bone marrow to recover, then another treatment, and so on. There is some debate about how many cycles of chemotherapy should be used in the treatment of colon and rectal cancer. Generally, after surgery, when patients receive chemotherapy alone for CRC, they are given between six to 12 cycles. When chemotherapy is administered along with radiation therapy, the patient may get two cycles before radiation therapy, two more cycles with the radiation therapy, and two to eight cycles after the radiation therapy. The exact schedule for chemotherapy depends on the specific drugs used. Some drugs may be given for a period of one week to two weeks, followed by a period of about 2 weeks to recover from the bone marrow suppressive effects.

Efficacy of 5-fluorouracil (5-FU) plus leucovorin (LV) as adjuvant chemotherapy for CRC has been established. Recently, large multicenter randomized controlled trials evaluating value of new regimens such as oxaliplatin+5-FU plus LV, capecitabine, and uracil-tegafur (UFT) plus LV, as compared with 5- FU plus LV, have been conducted. 
Although further integration of targeted compounds including cetuximab and bevacizumab into adjuvant therapy may be promising, cost issues have begun to emerge.

\subsection{Chemoprevention and Colorectal Cancer}

Chemoprevention is the use of drugs prophylactically to decrease the risk of developing cancer, reverse the pre-malignant process and re-regulate cellular growth and differentiation (Daniels and McKee, 1995). Currently, several drugs are thought to be effective chemopreventive agents in the fight against CRC. These medications include non-steroidal anti-inflammatory drugs (NSAIDs), such as aspirin, and COX-2 inhibitors. Epidemiological evidence establishes an association between NSAID use and the reduction in incidence of CRC (Suh, 1993; Muscat, 1994; Logan, 1993; Greenberg, 1993). For the most part, this association is based on observational studies. Unfortunately, interpretation of such studies may be misleading. Still, many experts will not rule out the possibility that aspirin has a positive impact on CRC prevention. Studies have also been conducted to assess the possibility of combination therapy. However, chemoprevention should not replace periodic screenings with methods such as FOBT, FS, and colonoscopy (Ladabaum, Chopra et al., 2001).

\subsubsection{Aspirin}

Aspirin and other similar NSAIDs are the most widely studied agents in the chemoprevention of CRC (Ladabaum, Chopra et al., 2001). This class of drugs has proven effective as modest chemopreventive agents. Investigators at Dartmouth Medical Center conducted a randomized, double-blinded study including 1,000 participants in 
three randomized groups. The three groups received either 81 milligrams or 325 milligrams of aspirin, or a placebo. It was found that there was about a 19\% reduction in polyp (precursor for CRC) recurrence in the low (81mg) aspirin dose group (Baron, Cole et al., 2003). Recent observations suggest that aspirin and other NSAIDs have a chemopreventive benefit. A case-control study demonstrated a 40-50\% reduction in the risk of adenomas and CRC among patients who took aspirin (Baron and Sandler, 2000). Two smaller case-control studies also demonstrated similar benefits with aspirin; but both lacked significant statistical power (Thun et al., 1991 and Thun et al., 1993).

Although aspirin has been found to provide a degree of protection against CRC, there is a risk of adverse side effects such as gastrointestinal ulceration. Aspirin is contraindicated in patients who have a history of gastrointestinal bleeds, and in those who have a triad of symptoms including asthma. In a 2001 study by Ladabaum and colleagues (Ladabaum et al., 2001), the authors reported that aspirin chemoprophylaxis alone remained generally non-cost-effective for those adhering to screening. They concluded that aspirin therapy should not substitute for screening; however aspirin may be used to supplement screening. When used as an adjunct to FOBT/FS, aspirin increased costs but decreased life-years due to related complications. Aspirin also yielded a small benefit in life-years as an adjunct to colonoscopy, but with a high incremental cost.

Chan and colleagues (Chan et al., 2005) suggest that optimal chemoprevention for CRC requires long-term use of aspirin at doses substantially higher than those recommended for prevention of cardiovascular disease. Nonaspirin NSAIDs appear to have a similar effect. However, the significant benefit of aspirin is not apparent until 
after a decade of use, with maximal risk reduction at doses greater than 14 tablets (325 milligrams) per week. However, the dose-related risk of gastrointestinal bleeding must also be considered.

\subsubsection{Cox-2 Inhibitors}

Enzymes known as cyclooxygenase 1 and 2 (COX-1 and COX-2) are important to the function of the human body. They both convert arachidonic acid to prostaglandins, which are then implicated in the mediation of pain, inflammation, cell proliferation and other key biologic responses. It has long been thought that COX-1 and COX-2 carry out the same biochemical reactions but that they are regulated differently that is, the switches for activating them are different. COX-1 inhibitors are simple, competitive, reversible inhibitors that compete with arachidonic acid for binding to the COX active site. The most commonly prescribed drugs in this class are ibuprofen, piroxicam, sulindac, and naproxen.

COX-2 is induced during inflammation and tissue repair. The primary benefit of COX-2 inhibitors is their ability to spare COX-1 and preserve gastrointestinal health. Also, COX-2 appears to be associated with colorectal adenocarcinomas, in that more than $80 \%$ of colon cancers express COX-2, compared with normal tissue. COX-2 inhibitors have been shown to significantly inhibit both early and late stages of chemically induced CRC in rats (Reedy et al., 2000). However, human data is not yet available to support these findings. Additionally, a 2003 study by Tuynman and colleagues (Tuynman et al., 2003) determined that an anti-carcinogenic effect had been shown specifically in selective COX-2 inhibitors in animal models. It has also been found that selective COX- 
2 inhibitors have fewer adverse effects than the non-selective NSAIDs and are seen as promising chemopreventive agents.

A study in 2001 by Arguedas and colleagues (Arguedas et al., 2001) compared the therapy of COX-2 inhibitors with CRC surveillance using colonoscopy. The study employed a Markov model with states representing the natural history of adenomas after endoscopic polypectomy (O’Leary et al., 2004). The results suggest that long-term COX2 inhibition is a more expensive, yet more effective, therapy when compared with colonoscopic surveillance for prevention. Arguedas and colleagues (Arguedas, Heudebert et al., 2001) also reported that COX-2 chemoprevention for surveillance in average-risk post-polypectomy patients is a more expensive strategy compared to colonoscopic surveillance. Another study by Ladabaum and colleagues (Ladabaum, Scheiman et al., 2003) concluded that although COX-2 inhibitors used as an adjunct to screening may increase life expectancy, it does so at prohibitive costs $(\$ 233,300$ per life year saved).

At the end of September 2004, Merck \&. Co., Inc. announced a voluntary withdrawal of VIOXX (rofecoxib), its COX-2 inhibitor. The decision to immediately withdraw the product was based on new, three year data from a prospective, randomized, placebo-controlled clinical trial, the APPROVe (Adenomatous Polyp Prevention on VIOXX) trial. The trial, before being halted, was designed to evaluate the efficacy of VIOXX 25mg in preventing recurrence of colorectal polyps in patients with a history of colorectal adenomas. In this study, there was an increased relative risk for confirmed cardiovascular (CV) events, such as heart attack and stroke, beginning after 18 months of treatment in the patients taking VIOXX compared to those taking placebo. It may cause 
heart attack and stroke due to its effect on platelets. The results for the first 18 months of the APPROVe study did not show any increased risk of confirmed CV events on VIOXX, and in this respect are similar to the results of two placebo-controlled studies described in the current U.S. labeling for VIOXX.

The National Cancer Institute's (NCI) Adenoma Prevention with Celecoxib (APC) Trial is a clinical trial to determine if celecoxib, which inhibits the enzyme COX2, reduces the occurrence of new adenomas (precancerous polyps) in the colon and rectum of people who have already had such a polyp removed. The trial enrolled participants from late 1999 through February 2002 and was scheduled to be completed by Spring 2005. The use of celecoxib in the APC Trial was suspended on December 17, 2004 because analysis by an independent Data Safety and Monitoring Board (DSMB) showed that the risk of major fatal and non-fatal cardiovascular events (cardiovascular death, heart attack, stroke, or heart failure) was 2.5 times higher for participants taking the drug compared to those on a placebo. Investigators in the APC trial immediately suspended study drug use, although the participants are under observation for the planned remainder of the trial. A December 2004 analysis of a similar study sponsored by Pfizer, called the PreSAP cancer trial, did not show an increased risk of cardiovascular events. APC investigators recently published a full report of the analysis of cardiovascular events on the trial (Solomon, McMurray et al., 2005). In this analysis, celecoxib use for an average of almost 3 years was associated with a dose-related increased risk of serious cardiovascular events (cardiovascular death, heart attack, stroke, or heart failure).

The NCI, despite the revelations about rofecoxib and celecoxib, has not abandoned the idea of using COX-2 inhibitors to reduce patients' risk of CRC. The web 
site ClinicalTrials.gov showed in March 2005 that recruitment continued for an NCIsponsored study of celecoxib in the prevention of CRC in patients with familial adenomatous polyposis. The NCI is attempting to continue on with the celecoxib trials in high-risk cohorts because the potential benefits may outweigh potential risks. As for the NCI-sponsored studies of COX-2 inhibitors in patients at lower risk of colorectal adenoma, that line of research is not currently being evaluated. The NCI now focuses its chemoprevention efforts with COX-2 inhibitors on high-risk patients-those with a 40$100 \%$ lifetime risk of developing a specific cancer. One goal of NCI's continuing research is to identify patients with relatively low safety concerns from long-term exposure to COX-2 inhibitors who would benefit greatly from the reduction in cancer risk.

Recent studies conducted by New Zealand and American revealed that Celebrex (Pfizer's brand of celecoxib), increases the risk of heart attacks in patients and is not any safer than similar medications that have been withdrawn from the market (Caldwell et al., 2006). However, in 2005, the Food and Drug Administration (FDA) concluded that the benefits of Celebrex outweighed the risks, thus making it the only remaining Cox-2 inhibitor available on the market. The FDA did require Celebrex to carry a "black box" warning alerting consumers of the heightened risk of heart attacks.

A new study, which used data from six previous clinical trials of Celebrex involving more than 12,000 patients, employed a meta-analyses to provide an overall answer to the ongoing Celebrex controversy. Researchers compared Celebrex's cardiovascular risks with those of a placebo drug and with a class of common analgesics, which have also been associated with heart attacks and strokes. The study reported that 
the use of Celebrex more than doubled the risk of heart attacks compared to a placebo

and had a 1.88-fold increase in comparison with other painkillers (Caldwell, Aldington et al., 2006).

Researchers may be able to develop new drugs with fewer side effects once they better understand how COX-2 inhibitors fight cancer and why the drugs carry a cardiovascular risk. The drugs may work by other means in addition to inhibiting the COX-2 enzyme.

\subsection{Cost of Colorectal Cancer}

The estimated direct cost for the treatment of CRC in the United States is over $\$ 6.5$ billion annually (Schrag and Weeks, 1999). Among malignancies, this is second only to breast cancer at $\$ 6.6$ billion per year. In a study sponsored by the NCI, the U.S. Office of Technology Assessment (OTA) estimates that treating a case of early stage cancer (stages one and two) costs approximately $\$ 35,000$; while treating late stage cancer (stages three and four) adds an additional $\$ 10,000$ to the cost. According to the ACS, only $37 \%$ of colorectal cancers are currently detected at a localized stage (ACS, 2006). Thus, the overall cost of treating CRC is much higher than it would be if a greater percentage of cases were detected early. Proponents of CRC screening hope that if utilization of screening tests increases, the percentage of cancers detected at an earlier stage will also increase. This may then lead to a reduction in treatment costs. Compared with screening for other types of cancer, colonoscopy for CRC screening has been shown to be more cost-effective. Colonoscopy (every ten years) has an average estimated cost of approximately $\$ 12,000$ per life year saved as compared with breast cancer screening 
(\$22,000) and cervical cancer screening (\$250,000) (Pignone, 2002; Wagner, 1996;

Frazier, 2000; Khandker, 2000; Sonnenberg, 2000; Vijan, 2001).

According to the NCI and the $\mathrm{CDC}$, the direct costs associated with the treatment of CRC in 1999 dollars were $\$ 4.8$ billion (Colon Cancer Alliance, 2001). Emergency room visits and visits to hospital outpatient departments, about 50,000 and 350,000 respectively accounted for $\$ 309.9$ million of this figure. Approximately $\$ 19.5$ million was spent on the cost of drugs to treat CRC, excluding chemotherapeutic agents administered in the physician office setting. In addition, hospitalizations accounted for $\$ 3.1$ billion, physician fees for $\$ 1.1$ billion, and home health care, hospice care and skilled nursing cost \$341 million. Adjusting this total for inflation, the total direct costs were nearly \$6.4 billion in 2003.

The adjusted amount for indirect costs for 2003 was approximately \$130.1 million (Colon Cancer Alliance, 2001). There were approximately 2.3 million days of work lost in 1998, for a work loss equivalent of $\$ 70.9$ million in lost wages among the working-age population. In addition to lost wages, indirect costs may be higher when lost productivity due to morbidity is accounted for.

\subsection{Quality of Life}

Quality of life (QOL) has been defined by the World Health Organization (WHO) as an individual's perception of their position in life in the context of the culture and value systems in which they live and in relation to their goals, expectations, standards and concerns (WHO, 1998). From the time of diagnosis, the QOL of every cancer patient and survivor is affected in some way, whether it be physical or emotional well-being, social 
and personal relationships, spirituality, personal fulfillment, or leisure planning. These effects can disrupt patient relations with family and friends, and effect socialization, career, and financial stability. Screening, diagnosis, and treatment of CRC all have significant short and long-term effects on patients' QOL. These effects may include pain and discomfort from screening procedures or from having part of the colon resected during surgery. Also, patients may suffer from emotional stress from being diagnosed with CRC, or throughout treatment, including adverse effects from chemotherapy. Physical QOL effects, such as the pain or discomfort from screening, and/or follow-up procedures, may also help explain why colorectal screening rates are low.

Trentham-Dietz and colleagues (Trentham-Dietz et al., 2003) conducted a study of 726 Wisconsin women diagnosed with colorectal cancer during the period 1990-1991. A follow-up survey was given eight years later to the 443 surviving participants. The study concluded that over the long term, factors attributable to aging, body weight, and chronic medical conditions play more dominant roles in determining physical and mental health than factors related to the initial CRC diagnosis.

In decision analysis, strategies are evaluated by comparing the overall benefits expected from pursuing each strategy. This is known as the expected value. The expected value of each strategy is calculated by multiplying the value of outcomes (utilities) by their probability of occurrence. In this way, investigators can differentiate between various strategies and choose the optimal strategy or path of events. Utilities are values that represent an individual's preferences for specific outcomes or health states under uncertainty and provide an approach to incorporate health-related QOL in a decision model. Utilities are measured on a scale of 0 to 1 , with 0 representing death and 
1 representing a state of full or perfect health. In the model, utilities are used to place value on outcomes and can be derived from published literature, direct elicitation, or from expert opinion.

\section{Utility Scales}

Direct utility elicitation involves using scaling methods to collect data on utilities. Examples are standard gamble, time trade-off, rating scales, and the Health Utilities Index (HUI). Standard gamble involves measuring an individual's preferences between a certainty of one outcome and a gamble of two possible outcomes. Time trade-off involves asking subjects to consider the amount of time or life expectancy they would be willing to trade in order to survive in various health states. The HUI is used to describe health status and produce utility scores. The HUI assigns utilities by using the mutiattribute framework. This method breaks health states into domains (attributes), assigns a value for each domain, and calculates overall utility by multiplying together the utilities for each attribute.

\section{Measure of Outcome}

The quality adjusted life year (QALY) is the most commonly used measure of outcome for decision analysis. QALYs can be expressed as life expectancy in a particular health state, multiplied by the utility in that health state, combining both quantity and quality into a single measure. This makes it possible to compare interventions that extend life expectancy but may result in some decrease in QOL, against other interventions that improve the QOL without extending life expectancy. The value 
of QALYs is that they can provide a common unit that can be compared across different disease areas or treatment groups. In this study, comparison is made across different screening strategies for CRC. 


\subsection{Cost-Effectiveness}

Cost-effectiveness analysis (CEA) is a technique for comparing the value of various clinical strategies where both the costs and effects of strategies are examined. Generally, the effects are the outcomes of the proposed program or treatment which is being evaluated. When a new strategy is compared with current practice one may calculate the cost-effectiveness ratio:

$$
\text { Cost-Effectiveness ratio }=\frac{(\text { cost of new strategy })-(\text { cost of current practice })}{(\text { effect of new strategy })-(\text { effect of current practice })}
$$

This ratio measures the difference of costs and effects between current practice and a new strategy.

\subsubsection{Cost-Effectiveness Studies in CRC Screening}

A meta-analysis by Pignone and colleagues (Pignone et al., 2002) compiled results from five cost-effectiveness studies of CRC screening. Within the studies, various strategies had incremental cost-effectiveness ratios between \$5,000 and \$40,000 per life year saved (see Table 2-1). 
Table 2-1 Cost-effectiveness Ratios of Colorectal Cancer Screening Strategies in Dollars per Life-year Saved

\begin{tabular}{|l|l|l|l|l|l|}
\hline Study & $\begin{array}{c}\text { Wagner } \\
\mathbf{( 1 9 9 6 )}\end{array}$ & $\begin{array}{c}\text { Frazier } \\
\mathbf{( 2 0 0 0 )}\end{array}$ & $\begin{array}{c}\text { Khandker } \\
\mathbf{( 2 0 0 0 )}\end{array}$ & $\begin{array}{c}\text { Sonnenberg } \\
\mathbf{( 2 0 0 0 )}\end{array}$ & $\begin{array}{c}\text { Vijan } \\
\mathbf{( 2 0 0 1 )}\end{array}$ \\
\hline FOBT $^{*}$ (annually) & 11,725 & 17,805 & 13,656 & 10,463 & 5,691 \\
\hline FS $^{* *}$ (every 5 years) & 12,477 & 15,630 & 12,804 & 39,359 & 19,068 \\
\hline Combination FOBT* \& FS** $^{*}$ & 13,792 & 22,518 & 18,693 & - & 17,942 \\
\hline DCBE $^{+}$(every 5 years) & 11,168 & 21,712 & 25,624 & - & - \\
\hline Colonoscopy (every 10 years) $^{2}$ & 10,933 & 21,889 & 22,012 & 11,840 & 9,038 \\
\hline
\end{tabular}

All costs are adjusted to year 2000 U.S. dollars.

* Fecal Occult Blood Test

** Flexible Sigmoidoscopy

+ Double Contrast Barrium Enema 
Pignone and colleagues (Pignone et al., 2002) also conducted a systematic review of the cost-effectiveness of CRC screening for the USPSTF. The review concluded that screening for CRC using FOBT, FS, combination FOBT/FS, DCBE, or colonoscopy is cost-effective when compared to no screening, but no one screening strategy can considered to be the "gold standard. One limitation to this review was that there was not sufficient eveidence to determine the cause of the differences among the results of the examined studies. The authors were not able to determine if these inconsistent results arose from either differences of the values used in the models, or from the differing model structures.

In a study by Vijan and colleagues (Vijan et al., 2001), the cost-effectiveness ratio of each screening strategy included is less than $\$ 20,000$ per life-year gained across all levels of compliance that were evaluated. The study also suggests that the cost of colonoscopy and the proportion of cancers that arise from polyps are key factors in determining the cost-effectiveness of screening for CRC. It was found that for the strategy of FOBT/FS to be the optimal choice in screening, one of the following must occur: a) $50 \%$ of CRC must arise from polyps, the compliance rate of screening must be very high (75\% or greater), and costs for colonoscopy must be moderate; or b) $50 \%$ of CRC arises from polyps, the compliance rate is $50 \%$, and the cost of colonoscopy is $\$ 1,000$ or more. Vijan and colleagues (2001) believe that under normal circumstances, meeting all of these conditions is unlikely. In addition, the study notes that the benefits of endoscopic screening by colonoscopy has not been demonstrated in randomized controlled trials, and that the screening combination of FS and FOBT has not yet been 
evaluated in clinical studies. Thus, effectiveness estimates are soley based on CRC natural history, particulary evolution of a precancerous polyp to a carcinoma.

Sonnenberg and colleagues (Sonnenberg et al., 2000) found that FOBT is a costeffective option compared with no screening and that FS is an expensive alternative to FOBT. Also, colonoscopy was found to have a relatively modest incremental costeffectiveness when compared with both FOBT and no screening. Given low compliance rates, colonoscopy every ten years is the most cost-effective means of CRC screening due to the reduction of mortality at relatively low incremental costs. This study did not evaluate the strategies of DCBE or the combination of FOBT/FS. The design of this study's model reduced the complex natural history of CRC to a few essential states and avoided making assumptions regarding transitions. In addition, this study included a compliance component and patients who became noncompliant with screening were assumed to remain in that state for the remainder of their life.

The study by Frazier and colleagues (Frazier, Colditz et al., 2000) varied compliance rates of screening from $60 \%$ to $100 \%$. The authors concluded that CRC screening is as cost-effective as other forms of cancer screenings. FOBT/FS (1yr/5yr) was the most cost-effective strategy with an $80 \%$ reduction in mortality. In addition, a single screening at age 55 using colonoscopy was found to achieve a 30\%-50\% reduction in mortality. One limitation of this study is that sensitivity of FOBT initial and repeat tests were assumed to be alike. However, polyps as well as cancers may bleed intermittently, and other conditions may cause rectal bleeding. Therefore, an initial FOBT may be falsely negative or positive. The sensitivity of FOBT increases with the number of samples per stool and the number of stools sampled (USPSTF, 2002). 
In a study by Khandker and colleagues (Khandker, Dulski et al., 2000), FS every five years and annual FOBT were the two most cost-effective strategies. However, with low screening compliance, FOBT was less cost-effective. Also, colonoscopy every ten years was more cost-effective when the cost of colonoscopy was reduced. Due to the lack of definitive clinical studies on the kinetics of adenomatous polyps and growth and spread of CRC published in the literature, assumptions in this study represent a best guess. The study also cited the lack of definitive clinical studies on the effectiveness of the various screening tests.

A study by Wagner and colleagues (Wagner, Tunis et al., 1996) concluded that FS and DCBE were comparable and were more cost-effective than the other stategies evaluated. Additionally, another report concluded that FOBT, though similar in costeffectiveness, does not deliver sensitivity equal to that seen in the Minnesota FOBT clinical trials (Mandel, Bond et al., 1993). In that study, the sensitivity of FOBT was found to be $80.8 \%$ and 92.2 , no rehydration and rehydration of slides, respectively. As with many studies, probabilities and utilities in this study were based on available literature, expert opinion, or best guess assumptions. Additionally, the study recognizes that all CRC screening tests are, in some way, uncomfortable or unpleasant to patients and that patients' acceptability of screening tests should be incorporated in the model. In another study, O’Leary and colleagues (O’Leary, Olynyk et al., 2004) concluded that FS was the most efficient strategy in terms of cost per life-year saved, followed by colonoscopy and FOBT (approximately $\$ 11,832$, $\$ 29,005$ and $\$ 33,031$, respectively, in then current U.S. dollars). Colorectal cancer screening studies reviewed 
by the USPSTF (2002) indicate that CRC screening is likely to be cost-effective (less than $\$ 30,000$ per additional life year gained) regardless of the strategy chosen.

A study by Ness and colleagues (Ness, Holmes et al., 2000) found that one-time colonoscopic screening in adults between ages 50 and 54 had a cost-effectiveness ratio of less than $\$ 10,000$. Although screening for CRC appears to be cost-effective when compared with no screening, the study concluded that no one strategy was the most effective or had the most desirable cost-effectiveness ratio. The lack of generalizability of the base model assumptions in this study was a limitation. All of the assumptions in the models were based on prevailing literature opinion, each of which was controversial. Cost-effectiveness studies have also been performed to assess cost issues with chemopreventive drugs. A study by Ladabaum and colleagues (2003) concluded that COX-2 inhibitors incur substantially higher costs per life-year saved than any of the currently recommended screening strategies alone. Although COX-2 inhibitors have been shown to possibly increase life expectancy when used as an adjunct to colonoscopy (every 5 or 10 years), costs are shown to be higher due to the expense of the drug. 


\section{Methods}

\subsection{Model Structure}

A Markov model was constructed to simulate the natural history of colorectal cancer (CRC). Figure 3-1 shows the Markov model and the allowable transitions between health states. The Markov model was then employed in a decision analytical model using Data TreeAge ${ }^{\circledR}$ software to compare alternative CRC screening strategies. The following nine screening strategies are included in the model:

1) annual Fecal Occult Blood Test (FOBT)

2) annual FOBT plus aspirin

3) annual FOBT plus COX-2 inhibitor

4) annual FOBT plus Flexible Sigmoidoscopy (FS) plus 5 years

5) annual FOBT plus FS every five years plus aspirin

6) annual FOBT plus FS every five years plus COX-2 inhibitor

7) colonoscopy every 10 years

8) colonoscopy every 10 years plus aspirin

9) colonoscopy every 10 years plus COX-2 inhibitor

The first branch point on the decision tree (Figure 3-2) is a decision node indicating a choice for CRC screening strategy. Figure 3-3, Figure 3-4, and Figure 3-5 display the structure of the arms of the decision model. Many of the arms/strategies are similar; however, the probabilities of events employed in each arm/strategy are different. Figure 3-3 shows the FOBT arm of the decision tree. This arm is identical for strategies one, two, and three (FOBT, FOBT plus aspirin, FOBT plus Cox-2). The arms in the model for strategies four, five, and six (all FOBT plus FlexSig strategies) are identical 
(Figure 3-4), and the arms with colonoscopy as a strategy (either alone or with chemoprevention) are also very similar (arms seven, eight, and nine) (Figure 3-5). 
Figure 3-1 Allowable Transitions between Health States in Colorectal Cancer Screening

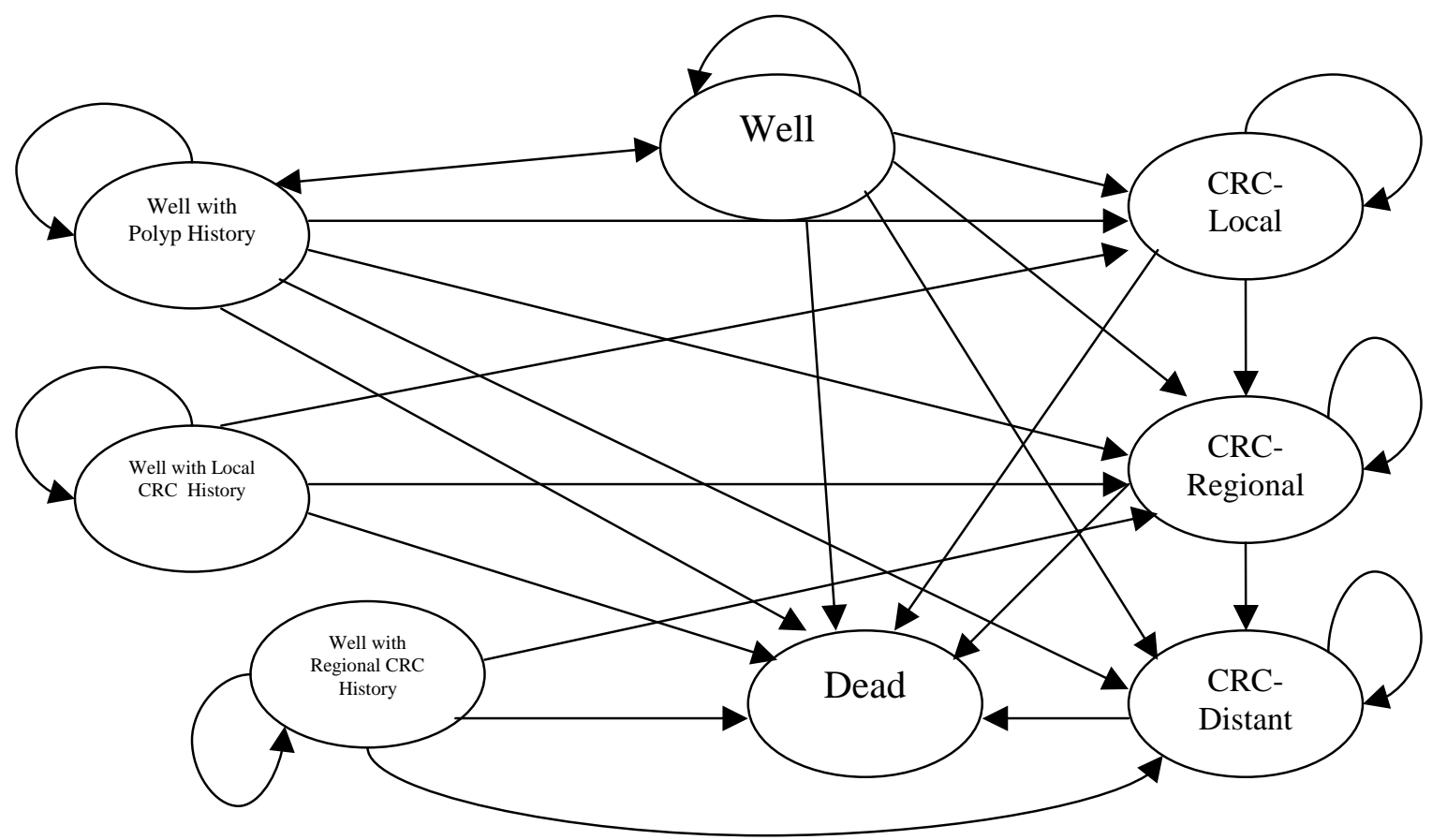


Figure 3-2 Collapsed Decision Tree of Colorectal Cancer Screening

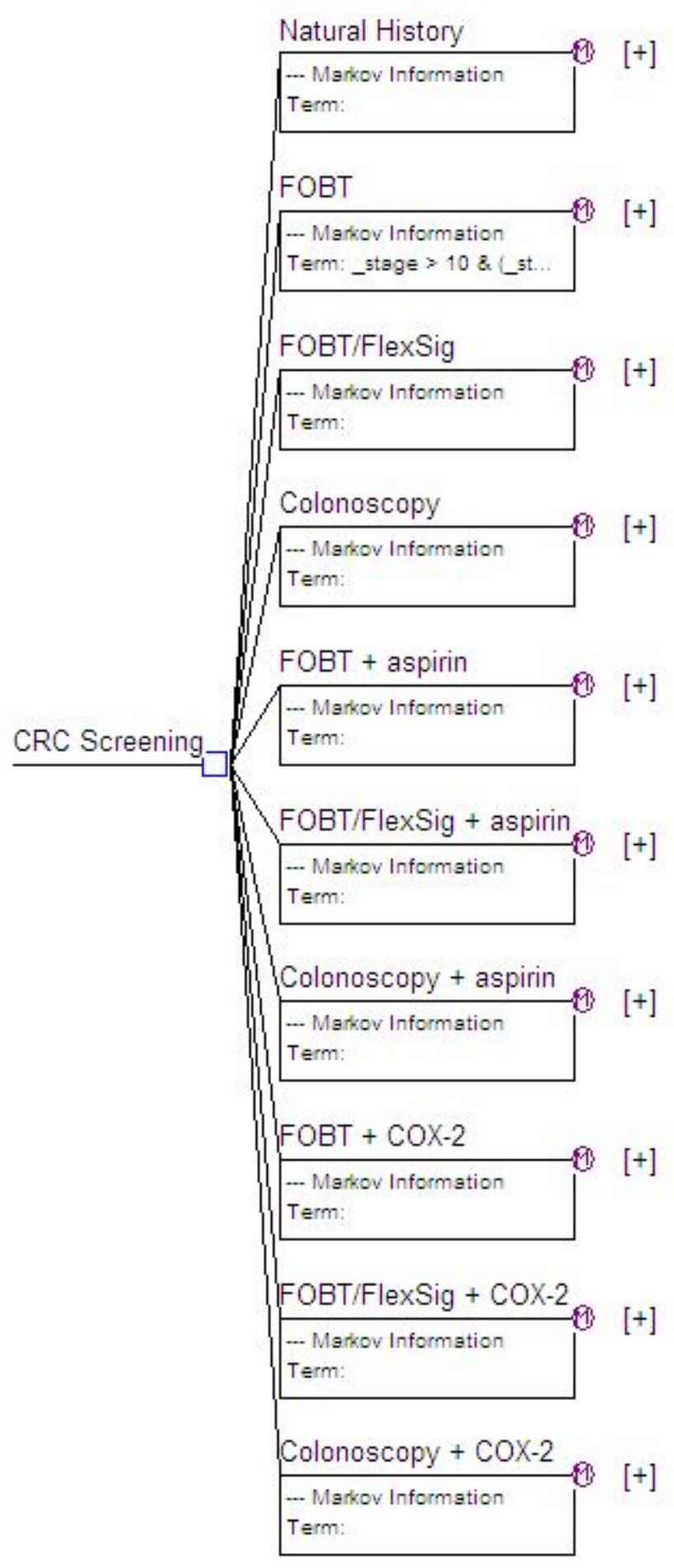


Figure 3-3 Structure of FOBT, FOBT plus Aspirin, and FOBT plus COX-2

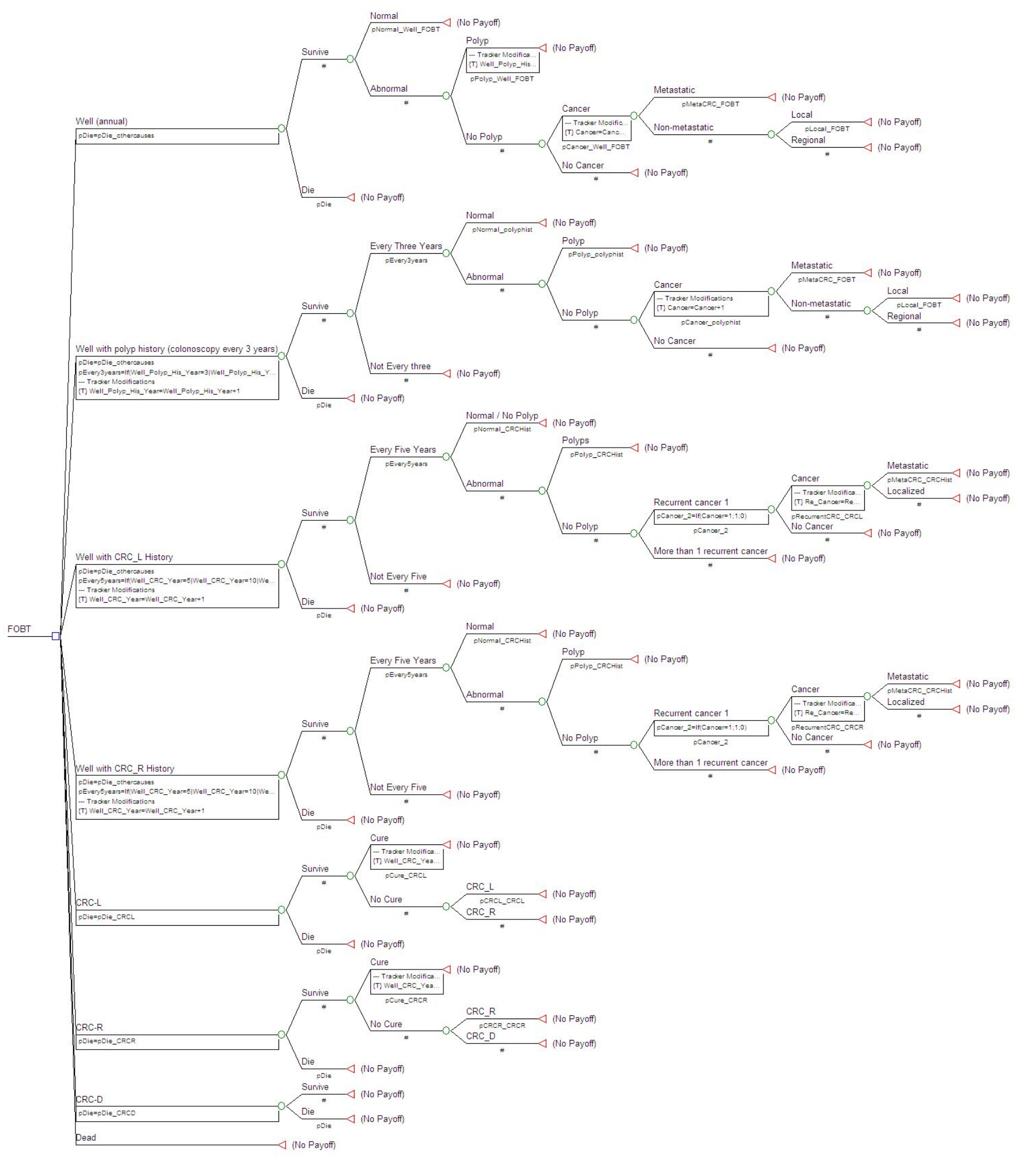


Figure 3-4 Structure of FOBT/FS, FOBT/FS plus Aspirin, and FOBT/FS plus COX-2

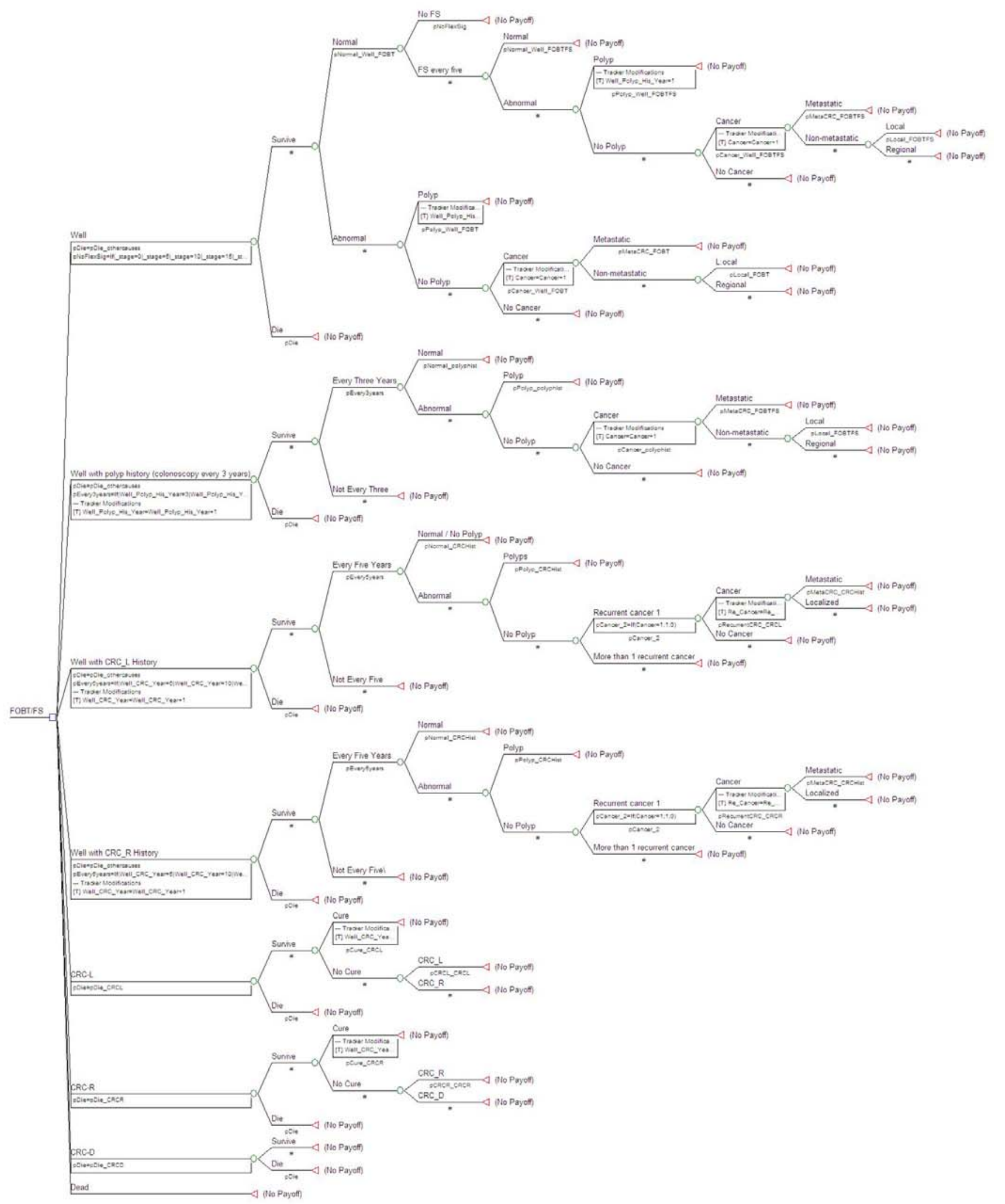


Figure 3-5 Structure of Colonoscopy, Colonoscopy plus Aspirin, and Colonoscopy plus COX-2

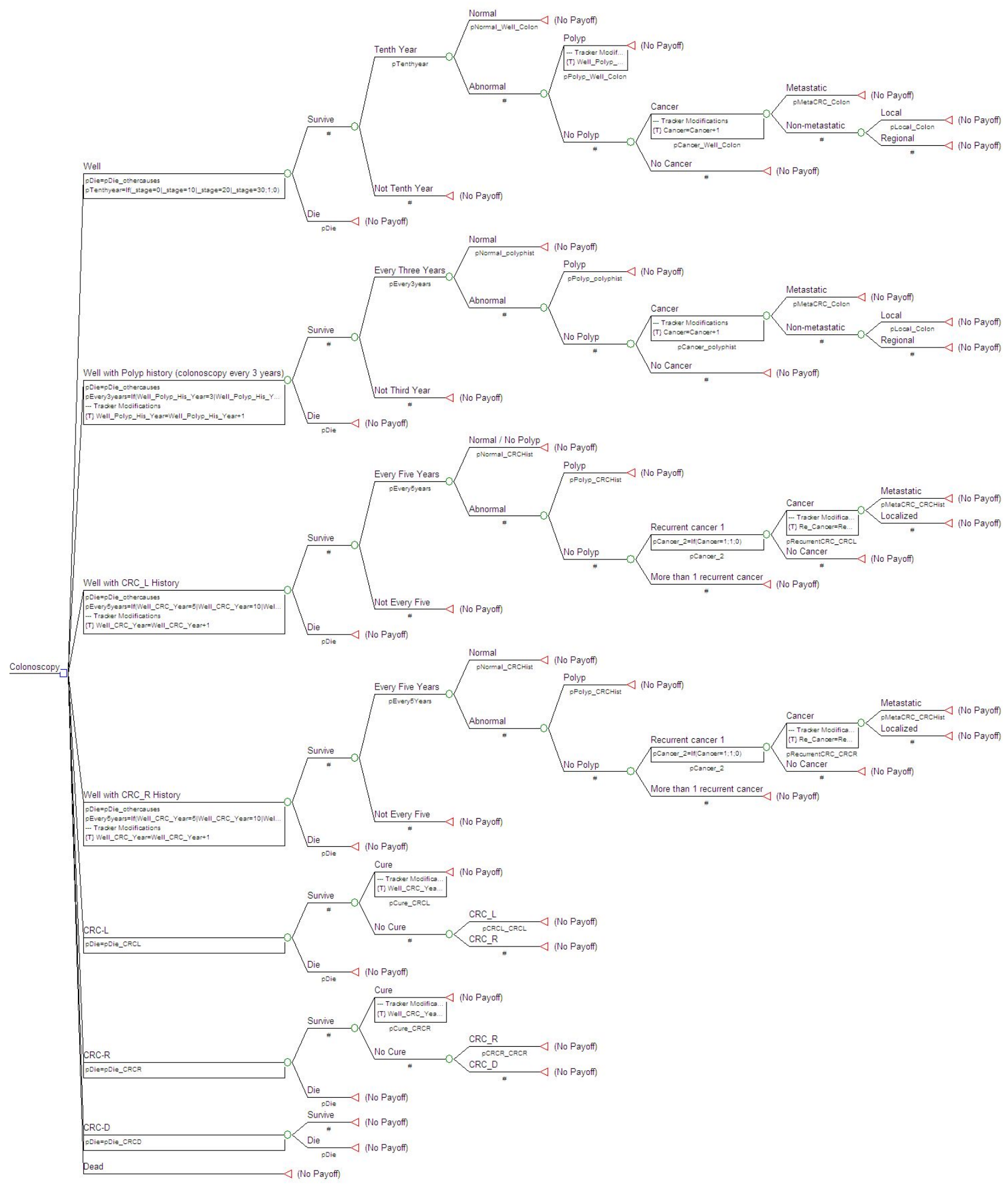




\subsection{Allowable Transitions}

A hypothetical cohort of 100,000 average-risk males 50 years of age was evaluated in the model. The model moved the cohort through different health states (Figure 3-1) for 35 cycle years or until death. A time horizon of 35 years was chosen since screening for CRC has been shown not to be cost-effective once an individual is 85 years of age. A Markov cycle is a period during which individuals can transition from one health state to another. The Markov cycle length was one year. That is, each individual remains in a health state for a full one-year cycle before transitioning into another state, or remaining in the same state. Transitions between health states are governed by probability values, known as transition probabilities. For example, if a patient is in the WELL state, after one year they may stay in the WELL state or move to the WELL WITH POLYP HISTORY, CRC-LOCAL, CRC-REGIONAL, CRCDISTANT or DEAD state. The DEAD state is an absorbing state, once an individual enters this state they cannot transition to another state. In reality, transitions occur at any time. However, in the model, they occur at discrete times. To best approximate the continuous reality, the assumption is made that transitions occur, on average, halfway through each cycle. Thus, we use half-cycle corrections to approximate the number in the middle of a cycle that begins halfway through the previous cycle and ends halfway through the subsequent cycle. This balances the over- and under-estimation.

\section{Screening Arm Structure}

In the FOBT arm, the entire cohort begins in the "Well" state. Then, an individual either survives or dies. Those individuals who die within a cycle year have 
died of causes other than CRC. If they survive, they would either have a normal (negative) or abnormal (positive) screening result. If the screening is normal, they remain in the "Well" state for the next cycle. If the screening is abnormal, a diagnostic colonoscopy is performed. During the diagnostic colonoscopy, if a polyp is found, a polypectomy is performed and the individual moves to the "Well with Polyp History" state for the next cycle. If no polyp is found, there is a possibility that cancer may be found. If cancer is not found, the abnormal (positive) test may have been a false positive and the individual would remain in the "Well" state for the next cycle. If it is cancer, it is either "Metastatic" or "Non-metastatic" and the individual will move to the corresponding state (CRC-D for metastatic; CRC-L or CRC-R for local or regional, respectively).

Upon entering the "Well with Polyp History" state, an individual will either survive or die. A surveillance colonoscopy is performed every 3 years after a polypectomy. If no polyp is found after the first surveillance colonoscopy, the individual will return to the "Well” state. If the colonoscopy returns an abnormal result (positive), either a polyp or cancer will be found and the individual will move to the corresponding state.

In the Local (CRC-L) and Regional (CRC-R) cancer states, an individual will either survive or die. After surviving, assuming 100\% treatment rate, either the patient will be "cured" or "not cured". If "cured", the individual will move to the "Well with CRC_L (or CRC_R) History” state. If the individual is not cured, they will remain in the current state or progress to the next cancer state. In the “CRC_D” state, an individual either survives or dies. 
After being cured of cancer, individuals enter either the "Well with CRC_L History" state or the "Well with CRC_R History" state. In these states, the individual either survives or dies. Upon surviving, a surveillance colonoscopy is performed every five years to check for recurrent cancer. If the colonoscopy is "normal" the individual stays in the current state. Otherwise, either a polyp or cancer will be found and the individual will move to the corresponding state.

All nine of the strategy arms move the cohort through the model in a similar way as that described above for the FOBT arm. However, the length of time an individual is in a state varies due to the different screening intervals for different screening strategies. In the FOBT/FS arm individuals have an annual FOBT then a follow-up FS every five years if the FOBT is "normal". If an FOBT is "abnormal" at any time, a diagnostic colonoscopy would be performed. A colonoscopy is performed every ten years for those strategies employing it as the screening procedure.

\subsection{Baseline Probabilities and Utilities}

The probabilities of various events and associated costs were the two types of data required to populate the decision analysis model. Probabilities of various events in the model were obtained through a review of the published literature, such as Ness and colleagues (Ness at al., 1998), Wagner and colleagues (Wagner et al., 1996), and Khandker and colleagues (Khandker at al., 2000). Both Khandker and colleagues and Wagner and colleagues conducted cost-effectiveness evaluations of three of the screening strategies used in this study (FOBT, FOBT plus FS, and colonoscopy). Previous studies have been performed to assess the effectiveness of aspirin (Ladabaum, Chopra et al., 
2001; Sandler et al., 2003) and COX-2 inhibitors (Steinbach, 2000, Arguedas 2001, Rahme 2003) as chemopreventive agents for CRC. Estimate values for each variable used in the decision tree were selected from such studies (see Table 3-1).

Incidence and mortality rates of CRC from the American Cancer Society (ACS) and the Center for Disease Control and Prevention (CDC) were also used. Transition rates/probabilities were estimated from U.S. vital statistics and cancer statistics and from published data on the sensitivity, specificity, and efficacy of various screening strategies. Also, survival data was obtained from the report of the National Cancer Institute's Surveillance, Epidemiology, and End Results (SEER, 2004). A description of each parameter, a baseline value, references for this value, and a range of values for sensitivity analysis is included in Table 3-1. 
Table 3-1 - Variables Used in Decision Analysis Model

\begin{tabular}{|c|c|c|}
\hline Variable & Value & Reference \\
\hline pCancer & 0.0025 & Winawer 1997 \\
\hline pCancer_CRCHist & 0.012 & Winawer 1997 \\
\hline pCancer_CRCHist_ASA & 0.008 & $\begin{array}{l}\text { Winawer 1997, Ladabaum } \\
2001\end{array}$ \\
\hline pCancer_CRCHist_COX2 & 0.008 & Winawer 1997, Steinbach 2000 \\
\hline pCancer_polyphist & 0.003 & Winawer 1997 \\
\hline pCancer_polyphist_ASA & 0.002 & Winawer 1997 \\
\hline pCancer_polyphist_COX2 & 0.002 & Winawer 1997 \\
\hline pCancer_Well_Colon & 0.0048 & Winawer 1997 \\
\hline pCancer_Well_Colon_ASA & 0.00336 & Winawer 1997, Ladabaum 2001 \\
\hline pCancer_Well_Colon_COX2 & 0.003456 & Winawer 1997, Steinbach 2000 \\
\hline pCancer_Well_FOBT & 0.0135 & Winawer 1997 \\
\hline pCancer_Well_FOBTFS & 0.00956 & Winawer 1997 \\
\hline pCancer_Well_FOBTFS_ASA & 0.00672 & Winawer 1997, Ladabaum 2001 \\
\hline pCancer_Well_FOBTFS_COX2 & 0.006912 & Winawer 1997 \\
\hline pCancer_Well_FOBT_ASA & 0.00945 & Winawer 1997, Ladabaum 2001 \\
\hline pCancer_Well_FOBT_COX2 & 0.00972 & Winawer 1997, Steinbach 2000 \\
\hline pCRCL_Well_FOBT & 0.7 & Winawer 1997 \\
\hline pLocal_Colon & 0.67 & Ladabaum 2001 \\
\hline pLocal_Colon_ASA & 0.64 & Ladabaum 2001 \\
\hline pLocal_Colon_COX2 & 0.66 & Ladabaum 2003 \\
\hline pLocal_CRCHist & 0.82 & Brady 1990 \\
\hline pLocal_FOBT & 0.7 & Ladabaum 2001 \\
\hline pLocal_FOBTFS & 0.735 & Ladabaum 2001 \\
\hline pLocal_FOBTFS_ASA & 0.69 & Ladabaum 2001 \\
\hline pLocal_FOBTFS_COX2 & 0.735 & Ladabaum 2003 \\
\hline pLocal_FOBT_ASA & 0.66 & Ladabaum 2001 \\
\hline pLocal_FOBT_COX2 & 0.7 & Ladabaum 2003 \\
\hline pMetaCRC_Colon & 0.118 & Ladabaum 01 \\
\hline pMetaCRC_Colon_ASA & 0.09 & Ladabaum 01 \\
\hline pMetaCRC_Colon_COX2 & 0.12 & Ladabaum 03 \\
\hline pMetaCRC_CRCHist & 0.18 & Brady 1990 \\
\hline pMetaCRC_FOBT & 0.1 & Ladabaum 01 \\
\hline pMetaCRC_FOBTFS & 0.106 & Ladabaum 01 \\
\hline pMetaCRC_FOBTFS_ASA & 0.08 & Ladabaum 01 \\
\hline pMetaCRC_FOBTFS_COX2 & 0.106 & Ladabaum 03 \\
\hline pMetaCRC_FOBT_ASA & 0.08 & Ladabaum 01 \\
\hline pMetaCRC_FOBT_COX2 & 0.1 & Ladabaum 03 \\
\hline pMetaCRC_Well_FOBT & 0.1 & Ladabaum 01 \\
\hline pNormal_CRCHist & 0.91 & $\begin{array}{l}\text { Rex 95, Weber 86, Larson 86, } \\
\text { Kronberg } 83, \text { Juhl } 90\end{array}$ \\
\hline pNormal_polyphist & 0.824 & $\begin{array}{l}\text { Rex 95, Winawer 97, Nava 87, } \\
\text { McFarland 91, Jorgenson } 93\end{array}$ \\
\hline pNormal_Well_Colon & 0.74 & Winawer 97 \\
\hline pNormal_Well_FOBTFS & 0.72 & Winawer 97 \\
\hline pNormal_Well_FOBT & 0.99 & Winawer 97 \\
\hline
\end{tabular}


Table 3-1 - Variables Used in Decision Analysis Model, continued

\begin{tabular}{|l|c|l|}
\hline pPolyp_CRCHist & 0.05 & McFarland 1991 \\
\hline pPolyp_CRCHist_ASA & 0.035 & \begin{tabular}{l} 
McFarland 1991, Ladabaum \\
\hline pPolyp_CRCHist_COX2
\end{tabular} \\
\hline pPolyp_polyphist & 0.036 & McFarland 1991, Rahme 2003 \\
\hline pPolyp_polyphist_ASA & 0.16 & Winawer 97 \\
\hline pPolyp_polyphist_COX2 & 0.112 & Winawer 97, Ladabaum 2001 \\
\hline pPolyp_Well_Colon & 0.115 & Winawer 97, Rahme 2003 \\
\hline pPolyp_Well_Colon_ASA & 0.29 & Winawer 97 \\
\hline pPolyp_Well_Colon_COX2 & 0.11991 & Winawer 97, Ladabaum 2001 \\
\hline pPolyp_Well_FOBT & 0.123336 & Winawer 97, Rahme 2003 \\
\hline pPolyp_Well_FOBTFS & 0.347 & Winawer 97 \\
\hline pPolyp_Well_FOBTFS_ASA & 0.14399 & Winawer 97, Ladabaum 2001 \\
\hline pPolyp_Well_FOBTFS_COX2 & 0.148104 & Winawer 97, Rahme 2003 \\
\hline pPolyp_Well_FOBT_ASA & 0.14693 & Winawer 97, Ladabaum 2001 \\
\hline pPolyp_Well_FOBT_COX2 & 0.151128 & Winawer 97, Rahme 2003 \\
\hline pRecurrentCRC_CRCL & 0.25 & Colorectal Cancer Network \\
\hline pRecurrentCRC_CRCR & 0.6 & Colorectal Cancer Network \\
\hline
\end{tabular}




\section{Utilities}

Utilities are numerical values assigned to health states. Utilities reflect the desirability of existing in a given state. Utilities range from 0 to 1 , with 1 representing perfect health and 0 representing death. Utilities for each health state are multiplied by the amount of time an individual spends in each state, and the results are added together over the individual's lifetime to obtain the estimated quality adjusted life years (QALYs). Utility values for the different Markov states used in this model were derived from published literature (Table 3-2). The utilities for local regional, and distant CRC were taken from Ness and colleagues (Ness et al., 1999). These are the utility values for individuals who have local, regional, and distant CRC. The utilities for individuals in the states of well with polyp history, well with history of local CRC, and well with history of regional CRC were estimated from the utilities in the Ness study. 
Table 3-2 - Utilities and their Corresponding Health States

\begin{tabular}{|l|l}
\hline uWell & 1 \\
\hline uPolypHist & $0.95^{\star *}$ \\
\hline uWell_LocalHist & $0.87^{\star *}$ \\
\hline uWell_RegionalHist & $0.84^{\star *}$ \\
\hline UCRC_L & $0.74^{\star}$ \\
\hline UCRC_R & $0.59^{*}$ \\
\hline uCRC_D & $0.25^{\star}$ \\
\hline uDead & 0 \\
\hline * Ness (1999) & \\
$* *$ estimated from Ness (1999) &
\end{tabular}




\subsection{Effectiveness and Costs}

Quality adjusted life years (QALYs) were used as the primary outcome measure. Costs were estimated from a societal perspective. The costs included were only direct costs. Wagner and colleagues (Wagner et al., 1996) Frazier and colleagues (Frazier et al., 2000), and Khandker and colleagues (Khandker et al., 2000) all included only direct costs from a societal perspective. Costs included cost of screening, diagnostic procedures, chemopreventive drugs, and costs for CRC treatment (localized, regional, and distant; initial and incremental). These costs were derived from Frazier and colleagues (Frazier et al., 2000) in which the costs were obtained from a large health maintenance organization (Taplin et al., 1995). The costs of CRC treatment include the actual costs of medical personnel and supplies to provide the service as well as overhead costs, such as medication administration, charting, and utilization of automated information systems. The costs of chemopreventive drugs were obtained using the average wholesale price (Redbook, 2005). All costs were updated to 2005 dollars using the medical care component of the Consumer Price Index (Statistical Abstract of the United States, 2005). Future health is often considered to be less valuable than immediate health. This was accounted for in the model by discounting future utility by a constant rate. The baseline discount rate in this model was $3 \%$. 


\section{Table 3-3 - Direct Costs of Colorectal Cancer Screening Procedures and Chemopreventive Agents}

\begin{tabular}{|c|c|c|c|c|c|}
\hline Variable & Description & $\begin{array}{l}\text { Cost in } \\
\text { dollars }\end{array}$ & Reference & $\begin{array}{c}\text { Low } \\
\text { Range } \\
\text { in dollars }\end{array}$ & $\begin{array}{c}\text { High } \\
\text { Range } \\
\text { in dollars } \\
\end{array}$ \\
\hline CASA & cost of $81 \mathrm{mg}$ of aspirin, 1 a day & 18 & Redbook & 14.4 & 21.6 \\
\hline cColonoscopy & cost of colonoscopy & $1,219.37$ & $\begin{array}{l}\text { Frazier 2000, Wagner 1996, } \\
\text { Khandker } 2000\end{array}$ & 975.5 & 1463.24 \\
\hline $\mathrm{cCOX} 2$ & cost of cyclooxygenas-2 inhibitor & $3,390.24$ & Redbook, 2005 & 2712.19 & 4068.29 \\
\hline cost of CRC Local & $\begin{array}{l}\text { Initial cost of treating localized } \\
\text { cancer (incremental) }\end{array}$ & $\begin{array}{r}16052 \\
(425) \\
\end{array}$ & Ness 2000 & NDA & NDA \\
\hline $\begin{array}{l}\text { cost of CRC } \\
\text { Regional }\end{array}$ & $\begin{array}{l}\text { Initial cost of treating regionalized } \\
\text { cancer (incremental) }\end{array}$ & $\begin{array}{l}18457 \\
(1944)\end{array}$ & Ness 2000 & NDA & NDA \\
\hline CFOBT & cost of fecal occult blood test & 45.79 & $\begin{array}{l}\text { Frazier 2000, Wagner 1996, } \\
\text { Khandker } 2000\end{array}$ & 36.63 & 54.95 \\
\hline $\mathrm{cFS}$ & cost of flexible sigmoidoscopy & 336.17 & $\begin{array}{l}\text { Frazier 2000, Wagner 1996, } \\
\text { Khandker } 2000\end{array}$ & 268.94 & 403.4 \\
\hline cPolypectomy & cost of polypectomy & 610.89 & $\begin{array}{l}\text { Frazier } 2000 \text {, Wagner } 1996 \text {, } \\
\text { Khandker } 2000\end{array}$ & 488.71 & 733.07 \\
\hline cost of CRC Distant & $\begin{array}{l}\text { Initial cost of treating metastatic } \\
\text { cancer (incremental) }\end{array}$ & $\begin{array}{c}21093 \\
(21,209)\end{array}$ & Ness 2000 & NDA & NDA \\
\hline
\end{tabular}

$\mathrm{c}=\mathrm{cost}$

NDA $=$ No Data Available 


\subsection{Outcome Measure}

Cost per QALY was used as the outcome measure in this study. The QALY combines quality of life with length of life by adjusting life-years with a quality weight, measured as a utility. Costs per life-year saved is the outcome measure employed in the cost-effectiveness studies used as references for this study. Currently, there is no costeffectiveness study analyzing the CRC screening procedures or chemopreventive agents used in this study in which the outcome measure is QALY.

\subsection{Base-case Cost-effectiveness Analysis}

The base case analysis represents the overall cost and effectiveness associated with each screening strategy. The costs and effectiveness obtained are then used to calculate the incremental cost-effectiveness ratio (ICER) for each screening strategy. The incremental analyses were performed by rank ordering the strategies by increasing cost after eliminating those that were more costly and less effective than an alternative strategy. In other words, those strategies that are more costly and less effective are ruled out by simple dominance. The ICER (additional cost divided by additional benefit) for each strategy was then calculated to compare with the next least expensive strategy. Strategies with a lower effectiveness, and higher cost-effectiveness ratios than another strategy, were ruled out by weak dominance and the ICER was then recalculated. 


\subsection{Sensitivity Analysis}

One-way sensitivity analyses were performed to assess the factors that have the greatest effect on the cost-effectiveness of screening. In this case, the sensitivity analyses were performed on all variables of interest in the model, including but not limited to screening strategies, as well as utility, or disutility, associated with screening. The analyses vary the variables over a range of values for each screening strategy to account for reasonable values for uncertain parameters. For example, the base case probability of developing localized cancer after an individual has previously been diagnosed and treated successfully for cancer (pRecurrentCRC_CRCL) is 0.25 . In the sensitivity analysis, the range for pRecurrentCRC_CRCL is 0.1 to 0.4 . The ranges of values for the sensitivity analyses were based on parameter values used in selected studies. One such study was

conducted by the U.S. Congress, Office of Technology Assessment (OTA). This study contains a summary of the specific assumptions about parameter values used in the analysis and the range of reasonable values for particularly uncertain parameters. The evidence for many of these assumptions is reviewed in OTA's previous report (OTA, 1990). 
Table 3-4 - Variables used in Sensitivity Analysis, Base Value, and Low and High Ranges

\begin{tabular}{|l|c|c|c|}
\hline Variable Name & Base Value & Low Range & High Range \\
\hline pCancer_Well_Colon_ASA & 0.00336 & 0.00312 & 0.0036 \\
\hline pCancer_Well_Colon_COX2 & 0.003456 & 0.003216 & 0.003696 \\
\hline pCancer_Well_FOBTFS_ASA & 0.00672 & 0.006214 & 0.00717 \\
\hline pCancer_Well_FOBTFS_COX2 & 0.006912 & 0.006405 & 0.0073612 \\
\hline pCancer_Well_FOBT_ASA & 0.00945 & 0.008775 & 0.010125 \\
\hline pCancer_Well_FOBT_COX2 & 0.00972 & 0.009045 & 0.010395 \\
\hline pNormal_CRCHist & 0.91 & 0.79 & 0.98 \\
\hline pNormal_polyphist & 0.824 & 0.75 & 0.88 \\
\hline pPolyp_Well_Colon_ASA & 0.11991 & 0.111345 & 0.128475 \\
\hline pPolyp_Well_Colon_COX2 & 0.123336 & 0.114771 & 0.131901 \\
\hline pPolyp_Well_FOBTFS_ASA & 0.14399 & 0.133705 & 0.154275 \\
\hline pPolyp_Well_FOBTFS_COX2 & 0.148104 & 0.137819 & 0.162589 \\
\hline pPolyp_Well_FOBT_ASA & 0.14693 & 0.136435 & 0.157425 \\
\hline pPolyp_Well_FOBT_COX2 & 0.151128 & 0.140633 & 0.161623 \\
\hline pRecurrentCRC_CRCL & 0.25 & 0.1 & 0.4 \\
\hline pRecurrentCRC_CRCR & 0.6 & 0.25 & 0.75 \\
\hline cASA & 18 & 14.4 & 21.6 \\
\hline cColonoscopy & $1,219.37$ & 975.5 & 1463.24 \\
\hline cCOX2 & $3,390.24$ & 2712.19 & 4068.29 \\
\hline cFOBT & 45.79 & 36.63 & 54.95 \\
\hline cFS & 336.17 & 268.94 & 403.4 \\
\hline cPolypectomy & 610.89 & 488.71 & 733.07 \\
\hline
\end{tabular}




\section{Results}

This study involved the construction of a decision analysis model to outline the costs and benefits associated with colorectal cancer (CRC) screening and the use of chemopreventive agents. This section presents the results of total costs, total effectiveness, and incremental cost-effectiveness ratios (ICER) of the different screening strategies incorporated in the decision model.

\subsection{Base Case Analysis Results}

The decision model was constructed to evaluate the total costs and benefits of nine CRC screening strategies (Figure 3-1). A hypothetical cohort of 100,000 averagerisk males was evaluated in the model. The base case results for cost, effectiveness and the ICER for each of the nine screening strategies are reported in Table 4-1. These results are also represented graphically in Figure 4-1.

The most cost-effective screening strategy was Fecal Occult Blood Test (FOBT); followed by FOBT plus aspirin, colonoscopy, and colonoscopy plus aspirin. The ICER of FOBT is $\$ 13,014.85$ compared to Natural History. The ICER of FOBT plus aspirin, the second most cost-effective strategy, is $\$ 7,173.35$ when compared to FOBT. The ICER of colonoscopy is $\$ 35.43$. This signifies that colonoscopy and FOBT plus aspirin are sensitive to changes in both cost and effectiveness. Small changes in either cost or effectiveness may alter the cost-effectiveness ordering of these two strategies. The least cost-effective strategies were those that utilized a COX-2 inhibitor as the chemopreventive agent.

The ICER results show the dominance status of the screening strategies. The five strategies at the bottom of Table 4-1 are all dominated by colonoscopy plus aspirin. This 
means that each of these strategies were more expensive and less effective than colonoscopy plus aspirin.

Figure 4-1 shows the cost-effectiveness of all nine screening strategies. The strategies toward the bottom of the graph were less expensive, while those to the right side of the graph were the most effective. The strategies that are the most cost-effective are represented in the bottom right quadrant of the graph. These include FOBT, FOBT plus aspirin, and colonoscopy. Those that are the represented in the upper portion of the graph represent the least cost-effective screening strategies. These include FOBT plus COX-2, FOBT/FS plus COX-2, and Colonoscopy plus COX-2. 
Table 4-1: Base Case Results of Colorectal Cancer Screening Strategies and Chemopreventive Agents

\begin{tabular}{lccc}
\hline Screening Strategy & $\begin{array}{c}\text { Costs } \\
\text { (in U.S. dollars) }\end{array}$ & Effectiveness & $\begin{array}{c}\text { Incremental Cost } \\
\text { Effectiveness Ratio }\end{array}$ \\
\hline Natural History & 188.02 & 16.8789 & -- \\
FOBT & 2295.9 & 17.0409 & $13,014.95$ \\
FOBT plus ASA & 2673.96 & 17.0936 & $7,173.55$ \\
Colonoscopy & 2677.6 & 17.1965 & 35.43 \\
Colonoscopy plus ASA & 3003.21 & 17.2083 & $27,513.64$ \\
FOBT plus FS & 3991.03 & 16.9322 & Dominated \\
FOBT/FS plus ASA & 4549.99 & 17.0091 & Dominated $^{\mathrm{a}}$ \\
FOBT plus COX-2 & $62,388.23$ & 17.0891 & Dominated $^{\mathrm{a}}$ \\
FOBT/FS plus COX-2 & $64,210.65$ & 17.0023 & Dominated $^{\mathrm{a}}$ \\
Colonoscopy plus COX-2 & $62,810.35$ & 17.2072 & Dominated $^{\mathrm{a}}$ \\
\hline
\end{tabular}

FOBT = annual Fecal Occult Blood Test

FOBT/FS - annual Fecal Occult Blood Test and Flexible Sigmoidoscopy every five years

Colonoscopy - Colonoscopy every ten years

ASA - aspirin, 81 milligrams daily

COX-2 - Cyclooxygenase-2 Inhibitor, 425 milligrams daily

aDominated by Colonoscopy plus ASA 
Figure 4-1 Cost-Effectiveness of Colorectal Cancer Screening and Chemoprevention

\section{Cost-Effectiveness Analysis At CRC Screening}

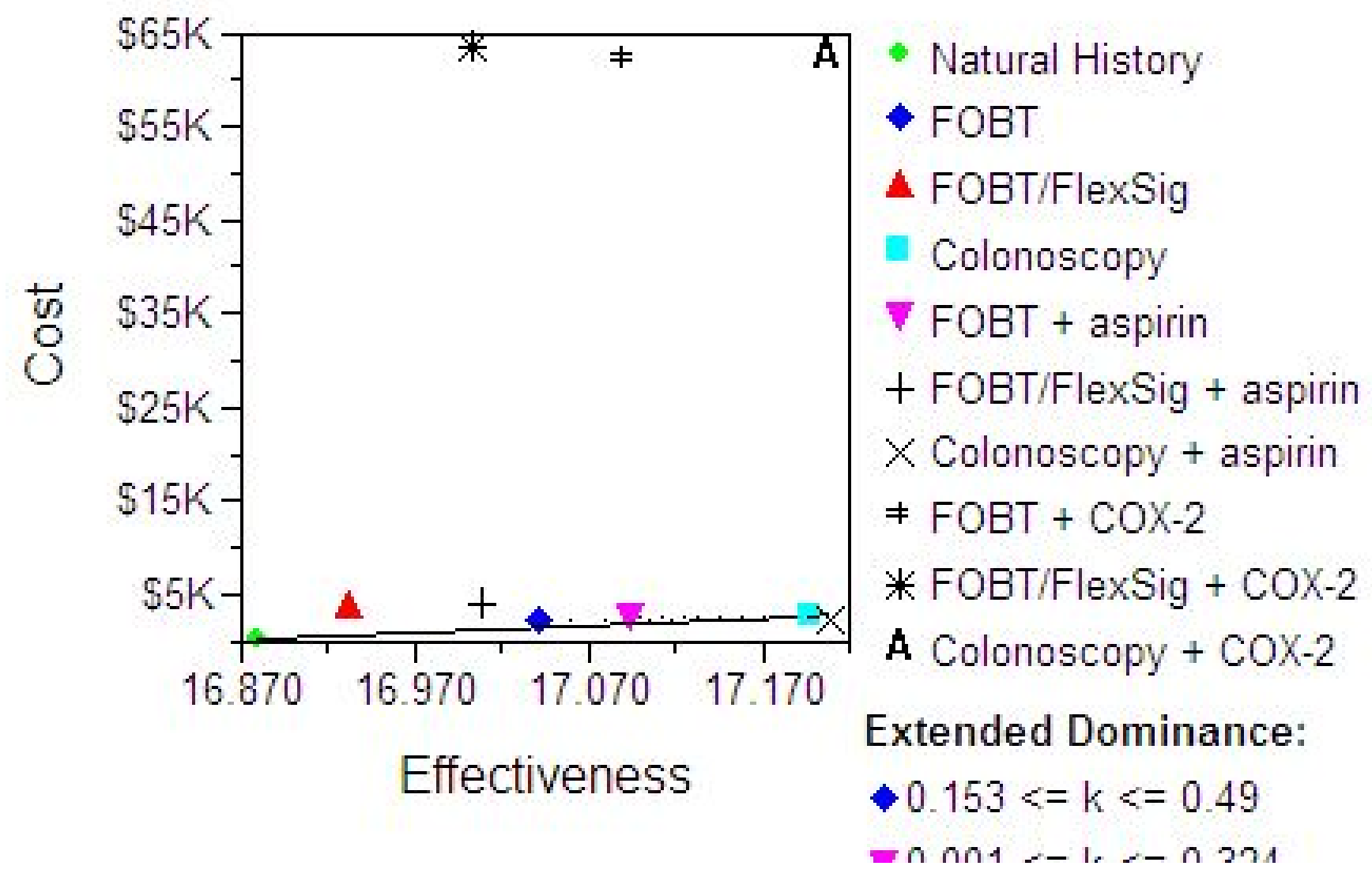




\subsection{Sensitivity Analysis}

The estimates for the input parameters, both costs and effectiveness were derived and integrated from multiple sources (Table 3-1 and Table 3-3). Thus, like any other economic model, the present model contains a measure of uncertainty. Sensitivity analysis is a commonly used tool to deal with uncertainty in the model input parameters. In one-way sensitivity analyses, one parameter at a time is varied over a certain range and the ICERs are re-calculated. A comparison between the original ICER and those obtained from sensitivity analyses provide an indication of the stability of the model to changes in the values of the parameter.

\section{Effect of Changes in Cost of Screening Strategies}

The impacts of changes in the cost of screening procedures are reported in Tables 4-2 through 4-5. The total costs the screening procedure were varied between plus and minus $20 \%$ of the baseline estimates.

The strategies of FOBT/Flexible Sigmoidoscopy (FS), FOBT/FS plus ASA, FOBT plus COX-2, colonoscopy plus COX-2, and FOBT/FS plus COX-2 all remained dominated by the strategy of colonoscopy plus ASA within all changes in parameters of cost. However, if the cost of FOBT was raised from $\$ 45.39$ to $\$ 46.03$ (\$0.64 difference), FOBT plus ASA would be dominated by colonoscopy. This is because colonoscopy is not only more effective, but also less expensive. In addition, if the cost of colonoscopy was reduced from $\$ 1,219.37$ to $\$ 1,215.57$ (\$3.80 difference), FOBT plus ASA would become dominated by colonoscopy (Table 4-4). This shows that the ICER is quite sensitive to the cost of these two screening procedures. The base case cost of 
polypectomy in this model is $\$ 610.89$. If the cost of polypectomy increased by approximately $\$ 30$ to $\$ 640.75$, FOBT plus ASA will again become dominated by colonoscopy (Table 4-5). The costs, effectiveness, and ICER results were not sensitive to changes in the cost of FS (Table 4-3).

Overall, the cost-effectiveness order did not change other than FOBT plus aspirin and colonoscopy when the above changes in cost parameters were made. 
Table 4-2: One Way Sensitivity Analyses Results of Colorectal Cancer Screening Strategies and Chemopreventive Agents Parameter: Cost of Fecal Occult Blood Test

\begin{tabular}{|c|c|c|c|c|c|c|c|c|c|}
\hline & Base Case: & $\$ 45.79$ & & Lower Range: & $\$ 36.63$ & & Upper Range: & $\$ 54.95$ & \\
\hline Screening Strategy & Cost & Effectiveness & ICER & Cost & Effectiveness & ICER & Cost & Effectiveness & ICER \\
\hline FOBT & $\$ 2,295.90$ & 17.041 & $\$ 13,014.95$ & $\$ 2,162.73$ & 17.041 & $\$ 12,192.71$ & $\$ 2,429.07$ & 17.041 & $\$ 13,837.19$ \\
\hline $\mathrm{FOBT}+\mathrm{ASA}$ & $\$ 2,673.96$ & 17.094 & $\$ 7,173.55$ & $\$ 2,532.94$ & 17.094 & $\$ 7,024.68$ & $\$ 2,814.97$ & 17.094 & (Dominated) $^{\mathrm{b}}$ \\
\hline Colonoscopy & $\$ 2,677.60$ & 17.196 & $\$ 35.43$ & $\$ 2,677.60$ & 17.196 & $\$ 1,405.62$ & $\$ 2,677.60$ & 17.196 & $\$ 1,597.09$ \\
\hline Colonoscopy + ASA & $\$ 3,003.21$ & 17.208 & $\$ 27,513.64$ & $\$ 3,003.21$ & 17.208 & $\$ 27,513.64$ & $\$ 3,003.21$ & 17.208 & $\$ 27,513.64$ \\
\hline FOBT/FS & $\$ 3,991.03$ & 16.932 & (Dominated) $^{a}$ & $\$ 3,875.01$ & 16.932 & ${\text { (Dominated })^{a}}^{a}$ & $\$ 4,107.05$ & 16.932 & (Dominated) $^{a}$ \\
\hline $\mathrm{FOBT} / \mathrm{FS}+\mathrm{ASA}$ & $\$ 4,549.99$ & 17.009 & ${\text { (Dominated })^{a}}^{a}$ & $\$ 4,422.31$ & 17.009 & ${\text { (Dominated })^{a}}^{a}$ & $\$ 4,677.68$ & 17.009 & ${\text { (Dominated })^{a}}^{a}$ \\
\hline $\mathrm{FOBT}+\mathrm{COX}-2$ & $\$ 62,388.23$ & 17.089 & (Dominated) $^{a}$ & $\$ 62,247.76$ & 17.089 & (Dominated) $^{a}$ & $\$ 62,528.70$ & 17.089 & (Dominated) $^{a}$ \\
\hline Colonoscopy + COX-2 & $\$ 62,810.35$ & 17.207 & (Dominated) $^{a}$ & $\$ 62,810.35$ & 17.207 & (Dominated) $^{a}$ & $\$ 62,810.35$ & 17.207 & (Dominated) $^{a}$ \\
\hline $\mathrm{FOBT} / \mathrm{FS}+\mathrm{COX}-2$ & $\$ 64,210.65$ & 17.002 & (Dominated) $^{a}$ & $\$ 64,083.79$ & 17.002 & (Dominated) $^{a}$ & $\$ 64,337.51$ & 17.002 & ${\text { (Dominated })^{a}}^{a}$ \\
\hline
\end{tabular}

FOBT = annual Fecal Occult Blood Test

FOBT/FS - annual Fecal Occult Blood Test and Flexible Sigmoidoscopy every five years

Colonoscopy - Colonoscopy every ten years

ASA - aspirin, 81 milligrams daily

COX-2 - Cyclooxygenase-2 Inhibitor, 425 milligrams daily

ICER = Incremental Cost-Effectiveness Ratio

aDominated by Colonoscopy plus ASA

${ }^{\mathrm{D}}$ Dominated by Colonoscopy 
Table 4-3: One Way Sensitivity Analyses Results of Colorectal Cancer Screening Strategies and Chemopreventive Agents Parameter: Cost of Flexible Sigmoidoscopy

\begin{tabular}{|c|c|c|c|c|c|c|c|c|c|}
\hline & Base Case: & $\$ 336.17$ & & Lower Range: & $\$ 268.94$ & & Upper Range: & $\$ 403.40$ & \\
\hline Screening Strategy & Cost & Effectiveness & ICER & Cost & Effectiveness & ICER & Cost & Effectiveness & ICER \\
\hline FOBT & $\$ 2,295.90$ & 17.041 & $\$ 13,014.95$ & $\$ 2,295.90$ & 17.041 & $\$ 13,014.95$ & $\$ 2,295.90$ & 17.041 & $\$ 13,014.95$ \\
\hline $\mathrm{FOBT}+\mathrm{ASA}$ & $\$ 2,673.96$ & 17.094 & $\$ 7,173.55$ & $\$ 2,673.96$ & 17.094 & $\$ 7,173.55$ & $\$ 2,673.96$ & 17.094 & $\$ 7,173.55$ \\
\hline Colonoscopy & $\$ 2,677.60$ & 17.196 & $\$ 35.43$ & $\$ 2,677.60$ & 17.196 & $\$ 35.43$ & $\$ 2,677.60$ & 17.196 & $\$ 35.43$ \\
\hline Colonoscopy + ASA & $\$ 3,003.21$ & 17.208 & $\$ 27,513.64$ & $\$ 3,003.21$ & 17.208 & $\$ 27,513.64$ & $\$ 3,003.21$ & 17.208 & $\$ 27,513.64$ \\
\hline FOBT/FS & $\$ 3,991.03$ & 16.932 & (Dominated) $^{\mathrm{a}}$ & $\$ 3,807.26$ & 16.932 & $(\text { Dominated })^{\mathrm{a}}$ & $\$ 4,174.80$ & 16.932 & (Dominated) ${ }^{\mathrm{a}}$ \\
\hline $\mathrm{FOBT} / \mathrm{FS}+\mathrm{ASA}$ & $\$ 4,549.99$ & 17.009 & (Dominated $^{\mathrm{a}}$ & $\$ 4,351.90$ & 17.009 & (Dominated $^{a}$ & $\$ 4,748.09$ & 17.009 & (Dominated) \\
\hline $\mathrm{FOBT}+\mathrm{COX}-2$ & $\$ 62,388.23$ & 17.089 & 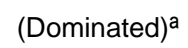 & $\$ 62,388.23$ & 17.089 & $(\text { Dominated })^{a}$ & $\$ 62,388.23$ & 17.089 & (Dominated) \\
\hline Colonoscopy + COX-2 & $\$ 62,810.35$ & 17.207 & $(\text { Dominated })^{\mathrm{a}}$ & $\$ 62,810.35$ & 17.207 & $(\text { Dominated })^{\mathrm{a}}$ & $\$ 62,810.35$ & 17.207 & (Dominated) $^{\mathrm{a}}$ \\
\hline FOBT/FS + COX-2 & $\$ 64,210.65$ & 17.002 & $(\text { Dominated })^{\mathrm{a}}$ & $\$ 64,013.57$ & 17.002 & (Dominated) $^{\mathrm{a}}$ & $\$ 64,407.73$ & 17.002 & (Dominated $^{\mathrm{a}}$ \\
\hline
\end{tabular}

FOBT = annual Fecal Occult Blood Test

FOBT/FS - annual Fecal Occult Blood Test and Flexible Sigmoidoscopy every five years

Colonoscopy - Colonoscopy every ten years

ASA - aspirin, 81 milligrams daily

COX-2 - Cyclooxygenase-2 Inhibitor, 425 milligrams daily

ICER = Incremental Cost-Effectiveness Ratio

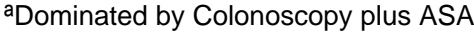


Table 4-4: One Way Sensitivity Analyses Results of Colorectal Cancer Screening Strategies and Chemopreventive Agents Parameter: Cost of Colonoscopy

\begin{tabular}{|c|c|c|c|c|c|c|c|c|c|}
\hline & Base Case: & $\$ 1,219.37$ & & Lower Range: & $\$ 975.50$ & & Upper Range: & $\$ 1,463.24$ & \\
\hline Screening Strategy & Cost & Effectiveness & ICER & Cost & Effectiveness & ICER & Cost & Effectiveness & ICER \\
\hline FOBT & $\$ 2,295.84$ & 17.041 & $\$ 13,014.60$ & $\$ 2,015.29$ & 17.041 & $\$ 11,282.37$ & $\$ 2,576.39$ & 17.041 & $\$ 14,746.82$ \\
\hline $\mathrm{FOBT}+\mathrm{ASA}$ & $\$ 2,673.89$ & 17.094 & $\$ 7,173.49$ & $\$ 2,377.09$ & 17.094 & (Dominated) $^{\mathrm{b}}$ & $\$ 2,970.69$ & 17.094 & $\$ 7,481.83$ \\
\hline Colonoscopy & $\$ 2,677.49$ & 17.196 & $\$ 34.97$ & $\$ 2,150.40$ & 17.196 & $\$ 868.20$ & $\$ 3,204.59$ & 17.196 & $\$ 2,272.67$ \\
\hline Colonoscopy + ASA & $\$ 3,003.10$ & 17.208 & $\$ 27,513.58$ & $\$ 2,472.37$ & 17.208 & $\$ 27,205.93$ & $\$ 3,533.84$ & 17.208 & $\$ 27,821.22$ \\
\hline FOBT/FS & $\$ 3,990.94$ & 16.932 & (Dominated) & $\$ 3,559.39$ & 16.932 & (Dominated) & $\$ 4,422.50$ & 16.932 & (Dominated) \\
\hline $\mathrm{FOBT} / \mathrm{FS}+\mathrm{ASA}$ & $\$ 4,549.90$ & 17.009 & (Dominated) & $\$ 4,079.54$ & 17.009 & (Dominated) & $\$ 5,020.25$ & 17.009 & (Dominated) \\
\hline $\mathrm{FOBT}+\mathrm{COX}-2$ & $\$ 62,388.17$ & 17.089 & (Dominated) & $\$ 62,092.49$ & 17.089 & (Dominated) & $\$ 62,683.84$ & 17.089 & (Dominated) \\
\hline Colonoscopy + COX-2 & $\$ 62,810.25$ & 17.207 & (Dominated) & $\$ 62,279.76$ & 17.207 & (Dominated) & $\$ 63,340.73$ & 17.207 & (Dominated) \\
\hline FOBT/FS + COX-2 & $\$ 64,210.55$ & 17.002 & (Dominated) & $\$ 63,742.94$ & 17.002 & (Dominated) & $\$ 64,678.16$ & 17.002 & (Dominated) \\
\hline
\end{tabular}

FOBT = annual Fecal Occult Blood Test

FOBT/FS - annual Fecal Occult Blood Test and Flexible Sigmoidoscopy every five years

Colonoscopy - Colonoscopy every ten years

ASA - aspirin, 81 milligrams daily

COX-2 - Cyclooxygenase-2 Inhibitor, 425 milligrams daily

ICER = Incremental Cost-Effectiveness Ratio

aDominated by Colonoscopy plus ASA

${ }^{\mathrm{b}}$ Dominated by Colonoscopy 
Table 4-5: One Way Sensitivity Analyses Results of Colorectal Cancer Screening Strategies and Chemopreventive Agents Parameter: Cost of Polypectomy

\begin{tabular}{|c|c|c|c|c|c|c|c|c|c|}
\hline & Base Case: & $\$ 610.89$ & & Lower Range: & $\$ 488.71$ & & Upper Range: & $\$ 733.07$ & \\
\hline Screening Strategy & Cost & Effectiveness & ICER & Cost & Effectiveness & ICER & Cost & Effectiveness & ICER \\
\hline FOBT & $\$ 2,295.90$ & 17.041 & $\$ 13,014.95$ & $\$ 2,266.40$ & 17.041 & $\$ 12,832.82$ & $\$ 2,325.40$ & 17.041 & $\$ 13,197.08$ \\
\hline $\mathrm{FOBT}+\mathrm{ASA}$ & $\$ 2,673.96$ & 17.094 & $\$ 7,173.55$ & $\$ 2,652.11$ & 17.094 & $\$ 7,318.77$ & $\$ 2,695.80$ & 17.094 & (Dominated) $^{\mathrm{b}}$ \\
\hline Colonoscopy & $\$ 2,677.60$ & 17.196 & $\$ 35.43$ & $\$ 2,670.68$ & 17.196 & $\$ 180.44$ & $\$ 2,684.52$ & 17.196 & $\$ 2,307.75$ \\
\hline Colonoscopy + ASA & $\$ 3,003.21$ & 17.208 & $\$ 27,513.64$ & $\$ 2,998.34$ & 17.208 & $\$ 27,686.23$ & $\$ 3,008.09$ & 17.208 & $\$ 27,341.06$ \\
\hline FOBT/FS & $\$ 3,991.03$ & 16.932 & (Dominated) $^{a}$ & $\$ 3,946.05$ & 16.932 & ${(\text { Dominated })^{a}}^{a}$ & $\$ 4,036.01$ & 16.932 & (Dominated) $^{\mathrm{a}}$ \\
\hline $\mathrm{FOBT} / \mathrm{FS}+\mathrm{ASA}$ & $\$ 4,549.99$ & 17.009 & ${\text { (Dominated })^{a}}^{a}$ & $\$ 4,515.67$ & 17.009 & $(\text { Dominated })^{a}$ & $\$ 4,584.31$ & 17.009 & $(\text { Dominated })^{a}$ \\
\hline $\mathrm{FOBT}+\mathrm{COX}-2$ & $\$ 62,388.23$ & 17.089 & (Dominated) $^{a}$ & $\$ 62,365.84$ & 17.089 & (Dominated) $^{a}$ & $\$ 62,410.61$ & 17.089 & (Dominated) $^{a}$ \\
\hline Colonoscopy + COX-2 & $\$ 62,810.35$ & 17.207 & (Dominated) $^{a}$ & $\$ 62,805.34$ & 17.207 & (Dominated) $^{a}$ & $\$ 62,815.37$ & 17.207 & (Dominated) $^{a}$ \\
\hline $\mathrm{FOBT} / \mathrm{FS}+\mathrm{COX}-2$ & $\$ 64,210.65$ & 17.002 & (Dominated) $^{a}$ & $\$ 64,175.55$ & 17.002 & (Dominated) $^{a}$ & $\$ 64,245.74$ & 17.002 & ${\text { (Dominated })^{a}}^{a}$ \\
\hline
\end{tabular}

FOBT = annual Fecal Occult Blood Test

FOBT/FS - annual Fecal Occult Blood Test and Flexible Sigmoidoscopy every five years

Colonoscopy - Colonoscopy every ten years

ASA - aspirin, 81 milligrams daily

COX-2 - Cyclooxygenase-2 Inhibitor, 425 milligrams daily

ICER = Incremental Cost-Effectiveness Ratio

aDominated by Colonoscopy plus ASA

${ }^{\mathrm{b}}$ Dominated by Colonoscopy 


\section{Effect of Changes in Cost of Chemopreventive Agents}

The impact of changes in the cost of chemopreventive agents is reported in Tables 4-6 and 4-7. The total costs of the chemopreventive agents were varied between plus $20 \%$ and minus $20 \%$ of the baseline estimates.

Similar to the sensitivity analyses for the costs of screening strategies, when the costs of chemopreventive agents are varied four strategies remain as cost-effective. Those strategies are FOBT, FOBT plus ASA, Colonoscopy and Colonoscopy plus ASA. The base case cost of aspirin is $\$ 18$ per year. If this cost increased to $\$ 18.21$, FOBT plus aspirin would become dominated by colonoscopy. This minor change in the cost of aspirin shows that the ICER of FOBT plus aspirin and colonoscopy are quite sensitive to this cost.

The cost of a cycooxygenase-2 (COX-2) inhibitor is prohibitive with regard to cost-effectiveness. Although the screening strategies that include a COX-2 inhibitor have the greatest effectiveness, the costs are disproportionately high. The base case cost of COX-2 inhibitor in this model is $\$ 3,390.24$ per year. The cost of COX-2 inhibitor was varied by plus and minus $20 \%$ of the base-case estimate. No changes occurred; therefore the cost of COX-2 inhibition is not sensitive in the $20 \%$ range. Only if the cost of a COX-2 inhibitor was reduced to below $\$ 92.40$ would the cost not be prohibitive. At this cost, FOBT plus COX-2 inhibition would have higher effectiveness and lower costs than FOBT/FS. Additionally, the cost of a COX-2 inhibitor would have to be lower than the cost of aspirin to be more cost-effective than any strategy utilizing aspirin as the chemopreventive agent. 
Table 4-6: One Way Sensitivity Analyses Results of Colorectal Cancer Screening Strategies and Chemopreventive Agents Parameter: Cost of Aspirin

\begin{tabular}{|c|c|c|c|c|c|c|c|c|c|}
\hline & Base Case: & $\$ 18.00$ & & Lower Range: & $\$ 14.40$ & & Upper Range: & $\$ 21.60$ & \\
\hline Screening Strategy & Cost & Effectiveness & ICER & Cost & Effectiveness & ICER & Cost & Effectiveness & ICER \\
\hline FOBT & $\$ 2,295.90$ & 17.041 & $\$ 13,014.95$ & $\$ 2,295.90$ & 17.041 & $\$ 13,014.95$ & $\$ 2,295.90$ & 17.041 & $\$ 13,014.95$ \\
\hline $\mathrm{FOBT}+\mathrm{ASA}$ & $\$ 2,673.96$ & 17.094 & $\$ 7,173.55$ & $\$ 2,610.20$ & 17.094 & $\$ 5,963.84$ & $\$ 2,737.71$ & 17.094 & (Dominated) $^{\mathrm{b}}$ \\
\hline Colonoscopy & $\$ 2,677.60$ & 17.196 & $\$ 35.43$ & $\$ 2,677.60$ & 17.196 & $\$ 654.90$ & $\$ 2,677.60$ & 17.196 & $\$ 2,452.83$ \\
\hline Colonoscopy + ASA & $\$ 3,003.21$ & 17.208 & $\$ 27,513.64$ & $\$ 2,939.37$ & 17.208 & $\$ 22,118.66$ & $\$ 3,067.06$ & 17.208 & $\$ 32,908.62$ \\
\hline FOBT/FS & $\$ 3,991.03$ & 16.932 & $(\text { Dominated })^{a}$ & $\$ 3,991.03$ & 16.932 & $(\text { Dominated })^{a}$ & $\$ 3,991.03$ & 16.932 & $(\text { Dominated })^{a}$ \\
\hline $\mathrm{FOBT} / \mathrm{FS}+\mathrm{ASA}$ & $\$ 4,549.99$ & 17.009 & $(\text { Dominated })^{a}$ & $\$ 4,486.28$ & 17.009 & $\left(\right.$ Dominated) ${ }^{a}$ & $\$ 4,613.70$ & 17.009 & $(\text { Dominated })^{a}$ \\
\hline $\mathrm{FOBT}+\mathrm{COX}-2$ & $\$ 62,388.23$ & 17.089 & $(\text { Dominated })^{a}$ & $\$ 62,388.23$ & 17.089 & $(\text { Dominated })^{a}$ & $\$ 62,388.23$ & 17.089 & $(\text { Dominated })^{a}$ \\
\hline Colonoscopy + COX-2 & $\$ 62,810.35$ & 17.207 & (Dominated) $^{a}$ & $\$ 62,810.35$ & 17.207 & $(\text { Dominated })^{a}$ & $\$ 62,810.35$ & 17.207 & (Dominated) $^{a}$ \\
\hline $\mathrm{FOBT} / \mathrm{FS}+\mathrm{COX}-2$ & $\$ 64,210.65$ & 17.002 & $(\text { Dominated })^{a}$ & $\$ 64,210.65$ & 17.002 & (Dominated) $^{a}$ & $\$ 64,210.65$ & 17.002 & (Dominated) $^{a}$ \\
\hline
\end{tabular}

FOBT = annual Fecal Occult Blood Test

FOBT/FS - annual Fecal Occult Blood Test and Flexible Sigmoidoscopy every five years

Colonoscopy - Colonoscopy every ten years

ASA - aspirin, 81 milligrams daily

COX-2 - Cyclooxygenase-2 Inhibitor, 425 milligrams daily

ICER = Incremental Cost-Effectiveness Ratio

aDominated by Colonoscopy plus ASA

${ }^{\mathrm{b}}$ Dominated by Colonoscopy 
Table 4-7: One Way Sensitivity Analyses Results of Colorectal Cancer Screening Strategies and Chemopreventive Agents Parameter: Cost of Cyclooxygenase-2 Inhibitor

\begin{tabular}{|c|c|c|c|c|c|c|c|c|c|}
\hline & Base Case: & $\$ 3,390.24$ & & Lower Range: & $\$ 2,712.19$ & & Upper Range: & $\$ 4,068.29$ & \\
\hline Screening Strategy & Cost & Effectiveness & ICER & Cost & Effectiveness & ICER & Cost & Effectiveness & ICER \\
\hline FOBT & $\$ 2,295.90$ & 17.041 & $\$ 13,014.95$ & $\$ 2,295.90$ & 17.041 & $\$ 13,014.95$ & $\$ 2,295.90$ & 17.041 & $\$ 13,014.95$ \\
\hline $\mathrm{FOBT}+\mathrm{ASA}$ & $\$ 2,673.96$ & 17.094 & $\$ 7,173.55$ & $\$ 2,673.96$ & 17.094 & $\$ 7,173.55$ & $\$ 2,673.96$ & 17.094 & $\$ 7,173.55$ \\
\hline Colonoscopy & $\$ 2,677.60$ & 17.196 & $\$ 35.43$ & $\$ 2,677.60$ & 17.196 & $\$ 35.43$ & $\$ 2,677.60$ & 17.196 & $\$ 35.43$ \\
\hline Colonoscopy + ASA & $\$ 3,003.21$ & 17.208 & $\$ 27,513.64$ & $\$ 3,003.21$ & 17.208 & $\$ 27,513.64$ & $\$ 3,003.21$ & 17.208 & $\$ 27,513.64$ \\
\hline FOBT/FS & $\$ 3,991.03$ & 16.932 & ${\text { (Dominated })^{a}}^{a}$ & $\$ 3,991.03$ & 16.932 & ${\text { (Dominated })^{a}}^{a}$ & $\$ 3,991.03$ & 16.932 & ${\text { (Dominated })^{a}}^{a}$ \\
\hline $\mathrm{FOBT} / \mathrm{FS}+\mathrm{ASA}$ & $\$ 4,549.99$ & 17.009 & (Dominated) $^{a}$ & $\$ 4,549.99$ & 17.009 & (Dominated) $^{a}$ & $\$ 4,549.99$ & 17.009 & $\left(\right.$ Dominated) ${ }^{a}$ \\
\hline $\mathrm{FOBT}+\mathrm{COX}-2$ & $\$ 62,388.23$ & 17.089 & (Dominated) $^{a}$ & $\$ 50,381.54$ & 17.089 & (Dominated $^{a}$ & $\$ 74,394.91$ & 17.089 & (Dominated $^{a}$ \\
\hline Colonoscopy + COX-2 & $\$ 62,810.35$ & 17.207 & (Dominated) $^{a}$ & $\$ 50,785.15$ & 17.207 & (Dominated) $^{a}$ & $\$ 74,835.55$ & 17.207 & (Dominated) $^{a}$ \\
\hline FOBT/FS + COX-2 & $\$ 64,210.65$ & 17.002 & (Dominated) $^{a}$ & $\$ 52,212.63$ & 17.002 & (Dominated) $^{a}$ & $\$ 76,208.66$ & 17.002 & (Dominated) $^{a}$ \\
\hline
\end{tabular}

FOBT = annual Fecal Occult Blood Test

FOBT/FS - annual Fecal Occult Blood Test and Flexible Sigmoidoscopy every five years

Colonoscopy - Colonoscopy every ten years

ASA - aspirin, 81 milligrams daily

COX-2 - Cyclooxygenase-2 Inhibitor, 425 milligrams daily

ICER = Incremental Cost-Effectiveness Ratio

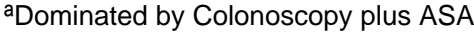


Effects of Changes in Probabilities

pNormal

The values of pNormal_CRCHist (Table 4-8) and pNormal_polyphist (Table 4-9) were varied over ranges specified in Table 3-1. These variables represent the probability of having a normal colonoscopy after having had a history of CRC and after having a history of polyps, respectively. When varied over the specified ranges, these two variables did not have any effect on the cost-effectiveness of the screening strategies, and the dominance status remained the same.

\section{pRecurrentCRC}

The variables pRecurrentCRC_CRCL (Table 4-10) and pRecurrentCRC_CRCR (Table 4-11) represent the probabilities of developing cancer after one has previously been diagnosed and treated successfully for cancer (local and regional, respectively). When varied over the specified ranges, these two variables had no effect on the costeffectiveness of the screening strategies, and the dominance status remained the same.

pPolyp_Well

The variable pPolyp_Well represents the probability of have a polyp found after an abnormal screening test while the individual is in the Well state. The variables included in the one-way sensitivity analysis with the "pPolyp_Well” prefix included

1) pPolyp_Well_FOBT_ASA (Table 4-12)

2) pPolyp_Well_FOBT_COX2 (Table 4-13) 
3) pPolyp_Well_FOBTFS_ASA (Table 4-14)

4) pPolyp_Well_FOBTFS_COX2 (Table 4-15)

5) pPolyp_Well_Colon_ASA (Table 4-16)

6) pPolyp_Well_Colon_COX2 (Table 4-17)

These six variables were varied over the ranges specified in Table 3-1. In the sensitivity analysis for pPolyp_Well_FOBT_ASA (Table 4-12), the variable (base-case 0.14693) was ranged from (0.136435) to (0.157425). When the probability of having a polyp detected after an abnormal screening test is lowered, the screening strategy FOBT plus aspirin becomes dominated by colonoscopy because the cost of FOBT plus aspirin exceeds the cost of colonoscopy, and the effectiveness remains lower.

In the sensitivity analysis for pPolyp_Well_Colon_ASA (Table 4-16), the screening strategy FOBT/FS plus Cox-2 becomes dominated by colonoscopy plus Cox-2 when the probability of detection of a polyp for colonoscopy plus aspirin is increased. In addition, when the probability of pPolyp_Well_Colon_COX2 (Table 4-17) is lowered to the lower range, the screening strategy FOBT/FS plus COX-2 becomes dominated by colonoscopy plus COX-2. This is due to the fact that the effectiveness of colonoscopy plus COX-2 slightly increases from baseline.

\section{pCancer_Well}

The variable pCancer_Well represents the probability of having cancer found after an abnormal screening test while the individual is in the Well state. The variables included in the one-way sensitivity analysis with the "pCancer_Well” prefix (Table 4-18 to Table 23) included: 
1) pCancer_Well_FOBT_ASA (Table 4-18)

2) pCancer_Well_FOBT_COX2 (Table 4-19)

3) pCancer_Well_FOBTFS_ASA (Table 4-20)

4) pCancer_Well_FOBTFS_COX2 (Table 4-21)

5) pCancer_Well_Colon_ASA (Table 4-22)

6) pCancer_Well_Colon_COX2 (Table 4-23)

When varied over the specified ranges in Table 3-1, these six variables had no effect on the cost-effectiveness of the screening strategies, and the dominance status remained the same. 
Table 4-8: One Way Sensitivity Analyses Results of Colorectal Cancer Screening Strategies and Chemopreventive Agents Parameter: pNormal_CRCHist

\begin{tabular}{|c|c|c|c|c|c|c|c|c|c|}
\hline & Base Case: & 0.91 & & Lower Range: & 0.79 & & Upper Range: & 0.98 & \\
\hline Screening Strategy & Cost & Effectiveness & ICER & Cost & Effectiveness & ICER & Cost & Effectiveness & ICER \\
\hline FOBT & $\$ 2,295.90$ & 17.041 & $\$ 13,014.95$ & $\$ 2,295.90$ & 17.041 & $\$ 13,014.95$ & $\$ 2,295.90$ & 17.041 & $\$ 13,014.95$ \\
\hline $\mathrm{FOBT}+\mathrm{ASA}$ & $\$ 2,673.96$ & 17.094 & $\$ 7,173.55$ & $\$ 2,673.96$ & 17.094 & $\$ 7,173.55$ & $\$ 2,673.96$ & 17.094 & $\$ 7,173.55$ \\
\hline Colonoscopy & $\$ 2,677.60$ & 17.196 & $\$ 35.43$ & $\$ 2,677.60$ & 17.196 & $\$ 35.43$ & $\$ 2,677.60$ & 17.196 & $\$ 35.43$ \\
\hline Colonoscopy + ASA & $\$ 3,003.21$ & 17.208 & $\$ 27,513.64$ & $\$ 3,003.21$ & 17.208 & $\$ 27,513.64$ & $\$ 3,003.21$ & 17.208 & $\$ 27,513.64$ \\
\hline FOBT/FS & $\$ 3,991.03$ & 16.932 & ${\text { (Dominated })^{a}}^{a}$ & $\$ 3,991.03$ & 16.932 & ${\text { (Dominated })^{a}}^{a}$ & $\$ 3,991.03$ & 16.932 & ${\text { (Dominated })^{a}}^{a}$ \\
\hline $\mathrm{FOBT} / \mathrm{FS}+\mathrm{ASA}$ & $\$ 4,549.99$ & 17.009 & (Dominated) $^{a}$ & $\$ 4,549.99$ & 17.009 & (Dominated) $^{a}$ & $\$ 4,549.99$ & 17.009 & ${\text { (Dominated })^{a}}^{a}$ \\
\hline $\mathrm{FOBT}+\mathrm{COX}-2$ & $\$ 62,388.23$ & 17.089 & (Dominated) $^{a}$ & $\$ 62,388.23$ & 17.089 & (Dominated $^{a}$ & $\$ 62,388.23$ & 17.089 & (Dominated $^{a}$ \\
\hline Colonoscopy + COX-2 & $\$ 62,810.35$ & 17.207 & (Dominated) $^{a}$ & $\$ 62,810.35$ & 17.207 & (Dominated) $^{a}$ & $\$ 62,810.35$ & 17.207 & (Dominated) $^{a}$ \\
\hline FOBT/FS + COX-2 & $\$ 64,210.65$ & 17.002 & (Dominated) $^{a}$ & $\$ 64,210.65$ & 17.002 & (Dominated) $^{a}$ & $\$ 64,210.65$ & 17.002 & (Dominated) $^{a}$ \\
\hline
\end{tabular}

FOBT = annual Fecal Occult Blood Test

FOBT/FS - annual Fecal Occult Blood Test and Flexible Sigmoidoscopy every five years

Colonoscopy - Colonoscopy every ten years

ASA - aspirin, 81 milligrams daily

COX-2 - Cyclooxygenase-2 Inhibitor, 425 milligrams daily

ICER = Incremental Cost-Effectiveness Ratio

aDominated by Colonoscopy plus ASA 
Table 4-9 One Way Sensitivity Analyses Results of Colorectal Cancer Screening Strategies and Chemopreventive Agents Parameter: pNormal_polyphist

\begin{tabular}{|c|c|c|c|c|c|c|c|c|c|}
\hline \multirow[b]{2}{*}{ Screening Strategy } & \multirow{2}{*}{$\begin{array}{l}\text { Base Case: } \\
\text { Cost }\end{array}$} & \multirow{2}{*}{$\begin{array}{c}0.824 \\
\text { Effectiveness }\end{array}$} & \multirow[b]{2}{*}{ ICER } & \multirow{2}{*}{$\begin{array}{c}\text { Lower Range: } \\
\text { Cost }\end{array}$} & \multirow{2}{*}{$\begin{array}{c}0.75 \\
\text { Effectiveness }\end{array}$} & \multirow[b]{2}{*}{ ICER } & \multicolumn{2}{|c|}{ Upper Range: 0.88} & \multirow[b]{2}{*}{ ICER } \\
\hline & & & & & & & Cost & Effectiveness & \\
\hline FOBT & $\$ 2,295.90$ & 17.041 & $\$ 13,014.95$ & $\$ 2,295.90$ & 17.041 & $\$ 13,014.95$ & $\$ 2,295.90$ & 17.041 & $\$ 13,014.95$ \\
\hline $\mathrm{FOBT}+\mathrm{ASA}$ & $\$ 2,673.96$ & 17.094 & $\$ 7,173.55$ & $\$ 2,673.96$ & 17.094 & $\$ 7,173.55$ & $\$ 2,673.96$ & 17.094 & $\$ 7,173.55$ \\
\hline Colonoscopy & $\$ 2,677.60$ & 17.196 & $\$ 35.43$ & $\$ 2,677.60$ & 17.196 & $\$ 35.43$ & $\$ 2,677.60$ & 17.196 & $\$ 35.43$ \\
\hline Colonoscopy + ASA & $\$ 3,003.21$ & 17.208 & $\$ 27,513.64$ & $\$ 3,003.21$ & 17.208 & $\$ 27,513.64$ & $\$ 3,003.21$ & 17.208 & $\$ 27,513.64$ \\
\hline FOBT/FS & $\$ 3,991.03$ & 16.932 & ${\text { (Dominated })^{a}}^{a}$ & $\$ 3,991.03$ & 16.932 & 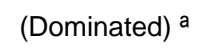 & $\$ 3,991.03$ & 16.932 & ${\text { (Dominated })^{a}}^{a}$ \\
\hline $\mathrm{FOBT} / \mathrm{FS}+\mathrm{ASA}$ & $\$ 4,549.99$ & 17.009 & (Dominated) $^{a}$ & $\$ 4,549.99$ & 17.009 & (Dominated) $^{a}$ & $\$ 4,549.99$ & 17.009 & (Dominated) $^{a}$ \\
\hline $\mathrm{FOBT}+\mathrm{COX}-2$ & $\$ 62,388.23$ & 17.089 & (Dominated) $^{a}$ & $\$ 62,388.23$ & 17.089 & 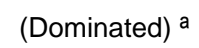 & $\$ 62,388.23$ & 17.089 & (Dominated $^{a}$ \\
\hline Colonoscopy + COX-2 & $\$ 62,810.35$ & 17.207 & (Dominated) $^{a}$ & $\$ 62,810.35$ & 17.207 & (Dominated) $^{a}$ & $\$ 62,810.35$ & 17.207 & ${\text { (Dominated })^{a}}^{a}$ \\
\hline $\mathrm{FOBT} / \mathrm{FS}+\mathrm{COX}-2$ & $\$ 64,210.65$ & 17.002 & (Dominated) $^{a}$ & $\$ 64,210.65$ & 17.002 & (Dominated) $^{a}$ & $\$ 64,210.65$ & 17.002 & (Dominated) $^{a}$ \\
\hline
\end{tabular}

FOBT = annual Fecal Occult Blood Test

FOBT/FS - annual Fecal Occult Blood Test and Flexible Sigmoidoscopy every five years

Colonoscopy - Colonoscopy every ten years

ASA - aspirin, 81 milligrams daily

COX-2 - Cyclooxygenase-2 Inhibitor, 425 milligrams daily

ICER = Incremental Cost-Effectiveness Ratio

aDominated by Colonoscopy plus ASA 
Table 4-10: One Way Sensitivity Analyses Results of Colorectal Cancer Screening Strategies and Chemopreventive Agents Parameter: pRecurrentCRC_CRCL

\begin{tabular}{|c|c|c|c|c|c|c|c|c|c|}
\hline & Base Case: & 0.25 & & Lower Range: & 0.1 & & Upper Range: & 0.4 & \\
\hline Screening Strategy & Cost & Effectiveness & ICER & Cost & Effectiveness & ICER & Cost & Effectiveness & ICER \\
\hline FOBT & $\$ 2,295.90$ & 17.041 & $\$ 13,014.95$ & $\$ 2,295.90$ & 17.041 & $\$ 13,014.95$ & $\$ 2,295.90$ & 17.041 & $\$ 13,014.95$ \\
\hline $\mathrm{FOBT}+\mathrm{ASA}$ & $\$ 2,673.96$ & 17.094 & $\$ 7,173.55$ & $\$ 2,673.96$ & 17.094 & $\$ 7,173.55$ & $\$ 2,673.96$ & 17.094 & $\$ 7,173.55$ \\
\hline Colonoscopy & $\$ 2,677.60$ & 17.196 & $\$ 35.43$ & $\$ 2,677.60$ & 17.196 & $\$ 35.43$ & $\$ 2,677.60$ & 17.196 & $\$ 35.43$ \\
\hline Colonoscopy + ASA & $\$ 3,003.21$ & 17.208 & $\$ 27,513.64$ & $\$ 3,003.21$ & 17.208 & $\$ 27,513.64$ & $\$ 3,003.21$ & 17.208 & $\$ 27,513.64$ \\
\hline FOBT/FS & $\$ 3,991.03$ & 16.932 & $(\text { Dominated })^{a}$ & $\$ 3,991.03$ & 16.932 & $(\text { Dominated })^{a}$ & $\$ 3,991.03$ & 16.932 & $(\text { Dominated })^{a}$ \\
\hline $\mathrm{FOBT} / \mathrm{FS}+\mathrm{ASA}$ & $\$ 4,549.99$ & 17.009 & (Dominated) $^{a}$ & $\$ 4,549.99$ & 17.009 & $(\text { Dominated })^{a}$ & $\$ 4,549.99$ & 17.009 & $(\text { Dominated })^{a}$ \\
\hline $\mathrm{FOBT}+\mathrm{COX}-2$ & $\$ 62,388.23$ & 17.089 & $\left(\right.$ Dominated) ${ }^{a}$ & $\$ 62,388.23$ & 17.089 & $(\text { Dominated })^{a}$ & $\$ 62,388.23$ & 17.089 & $(\text { Dominated })^{a}$ \\
\hline Colonoscopy + COX-2 & $\$ 62,810.35$ & 17.207 & $(\text { Dominated })^{a}$ & $\$ 62,810.35$ & 17.207 & $(\text { Dominated })^{a}$ & $\$ 62,810.35$ & 17.207 & ${\text { (Dominated })^{a}}^{a}$ \\
\hline FOBT/FS + COX-2 & $\$ 64,210.65$ & 17.002 & $(\text { Dominated })^{a}$ & $\$ 64,210.65$ & 17.002 & $(\text { Dominated })^{a}$ & $\$ 64,210.65$ & 17.002 & $(\text { Dominated })^{a}$ \\
\hline
\end{tabular}

FOBT = annual Fecal Occult Blood Test

FOBT/FS - annual Fecal Occult Blood Test and Flexible Sigmoidoscopy every five years

Colonoscopy - Colonoscopy every ten years

ASA - aspirin, 81 milligrams daily

COX-2 - Cyclooxygenase-2 Inhibitor, 425 milligrams daily

ICER = Incremental Cost-Effectiveness Ratio

aDominated by Colonoscopy plus ASA 
Table 4-11: One Way Sensitivity Analyses Results of Colorectal Cancer Screening Strategies and Chemopreventive Agents Parameter: pRecurrentCRC_CRCR

\begin{tabular}{|c|c|c|c|c|c|c|c|c|c|}
\hline & Base Case: & 0.6 & & Lower Range: & 0.25 & & Upper Range: & 0.75 & \\
\hline Screening Strategy & Cost & Effectiveness & ICER & Cost & Effectiveness & ICER & Cost & Effectiveness & ICER \\
\hline FOBT & $\$ 2,295.90$ & 17.041 & $\$ 13,014.95$ & $\$ 2,295.90$ & 17.041 & $\$ 13,014.95$ & $\$ 2,295.90$ & 17.041 & $\$ 13,014.95$ \\
\hline $\mathrm{FOBT}+\mathrm{ASA}$ & $\$ 2,673.96$ & 17.094 & $\$ 7,173.55$ & $\$ 2,673.96$ & 17.094 & $\$ 7,173.55$ & $\$ 2,673.96$ & 17.094 & $\$ 7,173.55$ \\
\hline Colonoscopy & $\$ 2,677.60$ & 17.196 & $\$ 35.43$ & $\$ 2,677.60$ & 17.196 & $\$ 35.43$ & $\$ 2,677.60$ & 17.196 & $\$ 35.43$ \\
\hline Colonoscopy + ASA & $\$ 3,003.21$ & 17.208 & $\$ 27,513.64$ & $\$ 3,003.21$ & 17.208 & $\$ 27,513.64$ & $\$ 3,003.21$ & 17.208 & $\$ 27,513.64$ \\
\hline FOBT/FS & $\$ 3,991.03$ & 16.932 & ${\text { (Dominated })^{a}}^{a}$ & $\$ 3,991.03$ & 16.932 & ${\text { (Dominated })^{a}}^{a}$ & $\$ 3,991.03$ & 16.932 & ${\text { (Dominated })^{a}}^{a}$ \\
\hline $\mathrm{FOBT} / \mathrm{FS}+\mathrm{ASA}$ & $\$ 4,549.99$ & 17.009 & (Dominated) $^{a}$ & $\$ 4,549.99$ & 17.009 & (Dominated) $^{a}$ & $\$ 4,549.99$ & 17.009 & ${\text { (Dominated })^{a}}^{a}$ \\
\hline $\mathrm{FOBT}+\mathrm{COX}-2$ & $\$ 62,388.23$ & 17.089 & (Dominated) $^{a}$ & $\$ 62,388.23$ & 17.089 & (Dominated $^{a}$ & $\$ 62,388.23$ & 17.089 & (Dominated $^{a}$ \\
\hline Colonoscopy + COX-2 & $\$ 62,810.35$ & 17.207 & (Dominated) $^{a}$ & $\$ 62,810.35$ & 17.207 & (Dominated) $^{a}$ & $\$ 62,810.35$ & 17.207 & (Dominated) $^{a}$ \\
\hline FOBT/FS + COX-2 & $\$ 64,210.65$ & 17.002 & (Dominated) $^{a}$ & $\$ 64,210.65$ & 17.002 & (Dominated) $^{a}$ & $\$ 64,210.65$ & 17.002 & (Dominated) $^{a}$ \\
\hline
\end{tabular}

FOBT = annual Fecal Occult Blood Test

FOBT/FS - annual Fecal Occult Blood Test and Flexible Sigmoidoscopy every five years

Colonoscopy - Colonoscopy every ten years

ASA - aspirin, 81 milligrams daily

COX-2 - Cyclooxygenase-2 Inhibitor, 425 milligrams daily

ICER = Incremental Cost-Effectiveness Ratio

aDominated by Colonoscopy plus ASA 
Table 4-12: One Way Sensitivity Analyses Results of Colorectal Cancer Screening Strategies and Chemopreventive Agents Parameter: pPolyp_Well_FOBT_ASA

\begin{tabular}{|c|c|c|c|c|c|c|c|c|c|}
\hline & Base Case: & 0.14693 & & Lower Range: & 0.136435 & & Upper Range: & 0.157425 & \\
\hline Screening Strategy & Cost & Effectiveness & ICER & Cost & Effectiveness & ICER & Cost & Effectiveness & ICER \\
\hline FOBT & $\$ 2,295.90$ & 17.041 & $\$ 13,014.95$ & $\$ 2,295.90$ & 17.041 & $\$ 13,014.95$ & $\$ 2,295.90$ & 17.041 & $\$ 13,014.95$ \\
\hline $\mathrm{FOBT}+\mathrm{ASA}$ & $\$ 2,673.96$ & 17.094 & $\$ 7,173.55$ & $\$ 2,688.22$ & 17.1 & (Dominated) $^{\mathrm{b}}$ & $\$ 2,659.86$ & 17.087 & $\$ 7,872.56$ \\
\hline Colonoscopy & $\$ 2,677.60$ & 17.196 & $\$ 35.43$ & $\$ 2,677.60$ & 17.196 & $\$ 2,452.83$ & $\$ 2,677.60$ & 17.196 & $\$ 162.19$ \\
\hline Colonoscopy + ASA & $\$ 3,003.21$ & 17.208 & $\$ 27,513.64$ & $\$ 3,003.21$ & 17.208 & $\$ 27,513.64$ & $\$ 3,003.21$ & 17.208 & $\$ 27,513.64$ \\
\hline FOBT/FS & $\$ 3,991.03$ & 16.932 & ${\text { (Dominated })^{a}}^{a}$ & $\$ 3,991.03$ & 16.932 & ${\text { (Dominated })^{a}}^{a}$ & $\$ 3,991.03$ & 16.932 & ${\text { (Dominated })^{a}}^{a}$ \\
\hline $\mathrm{FOBT} / \mathrm{FS}+\mathrm{ASA}$ & $\$ 4,549.99$ & 17.009 & (Dominated) $^{a}$ & $\$ 4,577.79$ & 17.015 & (Dominated) $^{a}$ & $\$ 4,522.55$ & 17.004 & ${\text { (Dominated })^{a}}^{a}$ \\
\hline $\mathrm{FOBT}+\mathrm{COX}-2$ & $\$ 62,388.23$ & 17.089 & (Dominated) $^{a}$ & $\$ 62,388.23$ & 17.089 & (Dominated $^{a}$ & $\$ 62,388.23$ & 17.089 & (Dominated $^{a}$ \\
\hline Colonoscopy + COX-2 & $\$ 62,810.35$ & 17.207 & (Dominated) $^{a}$ & $\$ 62,810.35$ & 17.207 & (Dominated) $^{a}$ & $\$ 62,810.35$ & 17.207 & (Dominated) $^{a}$ \\
\hline $\mathrm{FOBT} / \mathrm{FS}+\mathrm{COX}-2$ & $\$ 64,210.65$ & 17.002 & (Dominated) $^{a}$ & $\$ 64,210.65$ & 17.002 & (Dominated) $^{a}$ & $\$ 64,210.65$ & 17.002 & (Dominated) $^{a}$ \\
\hline
\end{tabular}

FOBT = annual Fecal Occult Blood Test

FOBT/FS - annual Fecal Occult Blood Test and Flexible Sigmoidoscopy every five years

Colonoscopy - Colonoscopy every ten years

ASA - aspirin, 81 milligrams daily

COX-2 - Cyclooxygenase-2 Inhibitor, 425 milligrams daily

ICER = Incremental Cost-Effectiveness Ratio

aDominated by Colonoscopy plus ASA

${ }^{\mathrm{b}}$ Dominated by Colonoscopy 
Table 4-13: One Way Sensitivity Analyses Results of Colorectal Cancer Screening Strategies and Chemopreventive Agents Parameter: pPolyp_Well_FOBT_COX2

\begin{tabular}{|c|c|c|c|c|c|c|c|c|c|}
\hline & Base Case: & 0.151128 & & Lower Range: & 0.140633 & & Upper Range: & 0.161623 & \\
\hline Screening Strategy & Cost & Effectiveness & ICER & Cost & Effectiveness & ICER & Cost & Effectiveness & ICER \\
\hline FOBT & $\$ 2,295.90$ & 17.041 & $\$ 13,014.95$ & $\$ 2,295.90$ & 17.041 & $\$ 13,014.95$ & $\$ 2,295.90$ & 17.041 & $\$ 13,014.95$ \\
\hline $\mathrm{FOBT}+\mathrm{ASA}$ & $\$ 2,673.96$ & 17.094 & $\$ 7,173.55$ & $\$ 2,673.96$ & 17.094 & $\$ 7,173.55$ & $\$ 2,673.96$ & 17.094 & $\$ 7,173.55$ \\
\hline Colonoscopy & $\$ 2,677.60$ & 17.196 & $\$ 35.43$ & $\$ 2,677.60$ & 17.196 & $\$ 35.43$ & $\$ 2,677.60$ & 17.196 & $\$ 35.43$ \\
\hline Colonoscopy + ASA & $\$ 3,003.21$ & 17.208 & $\$ 27,513.64$ & $\$ 3,003.21$ & 17.208 & $\$ 27,513.64$ & $\$ 3,003.21$ & 17.208 & $\$ 27,513.64$ \\
\hline FOBT/FS & $\$ 3,991.03$ & 16.932 & ${\text { (Dominated })^{a}}^{a}$ & $\$ 3,991.03$ & 16.932 & ${\text { (Dominated })^{a}}^{a}$ & $\$ 3,991.03$ & 16.932 & ${\text { (Dominated })^{a}}^{a}$ \\
\hline $\mathrm{FOBT} / \mathrm{FS}+\mathrm{ASA}$ & $\$ 4,549.99$ & 17.009 & (Dominated) $^{a}$ & $\$ 4,549.99$ & 17.009 & (Dominated) $^{a}$ & $\$ 4,549.99$ & 17.009 & ${\text { (Dominated })^{a}}^{a}$ \\
\hline $\mathrm{FOBT}+\mathrm{COX}-2$ & $\$ 62,388.23$ & 17.089 & (Dominated) $^{a}$ & $\$ 62,400.43$ & 17.096 & (Dominated $^{a}$ & $\$ 62,388.23$ & 17.089 & (Dominated $^{a}$ \\
\hline Colonoscopy + COX-2 & $\$ 62,810.35$ & 17.207 & (Dominated) $^{a}$ & $\$ 62,810.35$ & 17.207 & (Dominated) $^{a}$ & $\$ 62,810.35$ & 17.207 & (Dominated) $^{a}$ \\
\hline FOBT/FS + COX-2 & $\$ 64,210.65$ & 17.002 & (Dominated) $^{a}$ & $\$ 64,236.11$ & 17.008 & (Dominated) $^{a}$ & $\$ 64,210.65$ & 17.002 & (Dominated) $^{a}$ \\
\hline
\end{tabular}

FOBT = annual Fecal Occult Blood Test

FOBT/FS - annual Fecal Occult Blood Test and Flexible Sigmoidoscopy every five years

Colonoscopy - Colonoscopy every ten years

ASA - aspirin, 81 milligrams daily

COX-2 - Cyclooxygenase-2 Inhibitor, 425 milligrams daily

ICER = Incremental Cost-Effectiveness Ratio

aDominated by Colonoscopy plus ASA 
Table 4-14: One Way Sensitivity Analyses Results of Colorectal Cancer Screening Strategies and Chemopreventive Agents Parameter: pPolyp_Well_FOBTFS_ASA

\begin{tabular}{|c|c|c|c|c|c|c|c|c|c|}
\hline & Base Case: & 0.14399 & & Lower Range: & 0.133705 & & Upper Range: & 0.154275 & \\
\hline Screening Strategy & Cost & Effectiveness & ICER & Cost & Effectiveness & ICER & Cost & Effectiveness & ICER \\
\hline FOBT & $\$ 2,295.90$ & 17.041 & $\$ 13,014.95$ & $\$ 2,295.90$ & 17.041 & $\$ 13,014.95$ & $2,295.90$ & 17.041 & $\$ 13,014.95$ \\
\hline $\mathrm{FOBT}+\mathrm{ASA}$ & $\$ 2,673.96$ & 17.094 & $\$ 7,173.55$ & $\$ 2,673.96$ & 17.094 & $\$ 7,173.55$ & $2,673.96$ & 17.094 & $\$ 7,173.55$ \\
\hline Colonoscopy & $\$ 2,677.60$ & 17.196 & $\$ 35.43$ & $\$ 2,677.60$ & 17.196 & $\$ 35.43$ & $2,677.60$ & 17.196 & $\$ 35.43$ \\
\hline Colonoscopy + ASA & $\$ 3,003.21$ & 17.208 & $\$ 27,513.64$ & $\$ 3,003.21$ & 17.208 & $\$ 27,513.64$ & $3,003.21$ & 17.208 & $\$ 27,513.64$ \\
\hline FOBT/FS & $\$ 3,991.03$ & 16.932 & ${\text { (Dominated })^{a}}^{a}$ & $\$ 3,991.03$ & 16.932 & ${\text { (Dominated })^{a}}^{a}$ & $3,991.03$ & 16.932 & ${\text { (Dominated })^{a}}^{a}$ \\
\hline $\mathrm{FOBT} / \mathrm{FS}+\mathrm{ASA}$ & $\$ 4,549.99$ & 17.009 & (Dominated) $^{a}$ & $\$ 4,569.52$ & 17.013 & (Dominated) $^{a}$ & $4,530.59$ & 17.005 & ${\text { (Dominated })^{a}}^{a}$ \\
\hline $\mathrm{FOBT}+\mathrm{COX}-2$ & $\$ 62,388.23$ & 17.089 & (Dominated) $^{a}$ & $\$ 62,388.23$ & 17.089 & (Dominated $^{a}$ & $62,388.23$ & 17.089 & (Dominated $^{a}$ \\
\hline Colonoscopy + COX-2 & $\$ 62,810.35$ & 17.207 & (Dominated) $^{a}$ & $\$ 62,810.35$ & 17.207 & (Dominated) $^{a}$ & $62,810.35$ & 17.207 & (Dominated) $^{a}$ \\
\hline FOBT/FS + COX-2 & $\$ 64,210.65$ & 17.002 & (Dominated) $^{a}$ & $\$ 64,210.65$ & 17.002 & (Dominated) $^{a}$ & $64,210.65$ & 17.002 & (Dominated) $^{a}$ \\
\hline
\end{tabular}

FOBT = annual Fecal Occult Blood Test

FOBT/FS - annual Fecal Occult Blood Test and Flexible Sigmoidoscopy every five years

Colonoscopy - Colonoscopy every ten years

ASA - aspirin, 81 milligrams daily

COX-2 - Cyclooxygenase-2 Inhibitor, 425 milligrams daily

ICER = Incremental Cost-Effectiveness Ratio

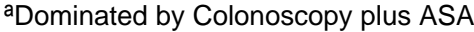


Table 4-15: One Way Sensitivity Analyses Results of Colorectal Cancer Screening Strategies and Chemopreventive Agents Parameter: pPolyp_Well_FOBTFS_COX2

\begin{tabular}{|c|c|c|c|c|c|c|c|c|c|}
\hline & Base Case: & 0.148104 & & Lower Range: & 0.137819 & & Upper Range: & 0.162589 & \\
\hline Screening Strategy & Cost & Effectiveness & ICER & Cost & Effectiveness & ICER & Cost & Effectiveness & ICER \\
\hline FOBT & $\$ 2,295.90$ & 17.041 & $\$ 13,014.95$ & $\$ 2,295.90$ & 17.041 & $\$ 13,014.95$ & $\$ 2,295.90$ & 17.041 & $\$ 13,014.95$ \\
\hline $\mathrm{FOBT}+\mathrm{ASA}$ & $\$ 2,673.96$ & 17.094 & $\$ 7,173.55$ & $\$ 2,673.96$ & 17.094 & $\$ 7,173.55$ & $\$ 2,673.96$ & 17.094 & $\$ 7,173.55$ \\
\hline Colonoscopy & $\$ 2,677.60$ & 17.196 & $\$ 35.43$ & $\$ 2,677.60$ & 17.196 & $\$ 35.43$ & $\$ 2,677.60$ & 17.196 & $\$ 35.43$ \\
\hline Colonoscopy + ASA & $\$ 3,003.21$ & 17.208 & $\$ 27,513.64$ & $\$ 3,003.21$ & 17.208 & $\$ 27,513.64$ & $\$ 3,003.21$ & 17.208 & $\$ 27,513.64$ \\
\hline FOBT/FS & $\$ 3,991.03$ & 16.932 & ${\text { (Dominated })^{a}}^{a}$ & $\$ 3,991.03$ & 16.932 & ${\text { (Dominated })^{a}}^{a}$ & $\$ 3,991.03$ & 16.932 & ${\text { (Dominated })^{a}}^{a}$ \\
\hline $\mathrm{FOBT} / \mathrm{FS}+\mathrm{ASA}$ & $\$ 4,549.99$ & 17.009 & (Dominated) $^{a}$ & $\$ 4,549.99$ & 17.009 & (Dominated) $^{a}$ & $\$ 4,549.99$ & 17.009 & $\left(\right.$ Dominated) ${ }^{a}$ \\
\hline $\mathrm{FOBT}+\mathrm{COX}-2$ & $\$ 62,388.23$ & 17.089 & (Dominated) $^{a}$ & $\$ 62,388.23$ & 17.089 & (Dominated $^{a}$ & $\$ 62,388.23$ & 17.089 & (Dominated $^{a}$ \\
\hline Colonoscopy + COX-2 & $\$ 62,810.35$ & 17.207 & (Dominated) $^{a}$ & $\$ 62,810.35$ & 17.207 & (Dominated) $^{a}$ & $\$ 62,810.35$ & 17.207 & (Dominated) $^{a}$ \\
\hline FOBT/FS + COX-2 & $\$ 64,206.97$ & 17.001 & (Dominated) $^{a}$ & $\$ 64,228.72$ & 17.007 & (Dominated) $^{a}$ & $\$ 64,185.38$ & 16.996 & (Dominated) $^{a}$ \\
\hline
\end{tabular}

FOBT = annual Fecal Occult Blood Test

FOBT/FS - annual Fecal Occult Blood Test and Flexible Sigmoidoscopy every five years

Colonoscopy - Colonoscopy every ten years

ASA - aspirin, 81 milligrams daily

COX-2 - Cyclooxygenase-2 Inhibitor, 425 milligrams daily

ICER = Incremental Cost-Effectiveness Ratio

aDominated by Colonoscopy plus ASA 
Table 4-16: One Way Sensitivity Analyses Results of Colorectal Cancer Screening Strategies and Chemopreventive Agents Parameter: pPolyp_Well_Colonoscopy_ASA

\begin{tabular}{|c|c|c|c|c|c|c|c|c|c|}
\hline & Base Case: & 0.11991 & & Lower Range: & 0.111345 & & Upper Range: & 0.128475 & \\
\hline Screening Strategy & Cost & Effectiveness & ICER & Cost & Effectiveness & ICER & Cost & Effectiveness & ICER \\
\hline FOBT & $\$ 2,295.90$ & 17.041 & $\$ 13,014.95$ & $\$ 2,295.90$ & 17.041 & $\$ 13,014.95$ & $\$ 2,295.90$ & 17.041 & $\$ 13,014.95$ \\
\hline $\mathrm{FOBT}+\mathrm{ASA}$ & $\$ 2,673.96$ & 17.094 & $\$ 7,173.55$ & $\$ 2,673.96$ & 17.094 & $\$ 7,173.55$ & $\$ 2,673.96$ & 17.094 & $\$ 7,173.55$ \\
\hline Colonoscopy & $\$ 2,677.60$ & 17.196 & $\$ 35.43$ & $\$ 2,677.60$ & 17.196 & $\$ 35.43$ & $\$ 2,677.60$ & 17.196 & $\$ 35.43$ \\
\hline Colonoscopy + ASA & $\$ 3,003.21$ & 17.208 & $\$ 27,513.64$ & $\$ 3,004.58$ & 17.21 & $\$ 23,923.52$ & $\$ 3,001.85$ & 17.206 & $\$ 32,410.73$ \\
\hline FOBT/FS & $\$ 3,991.03$ & 16.932 & ${\text { (Dominated })^{a}}^{a}$ & $\$ 3,991.03$ & 16.932 & ${\text { (Dominated })^{a}}^{a}$ & $\$ 3,991.03$ & 16.932 & (Dominated) $^{a}$ \\
\hline $\mathrm{FOBT} / \mathrm{FS}+\mathrm{ASA}$ & $\$ 4,549.99$ & 17.009 & (Dominated) $^{a}$ & $\$ 4,549.99$ & 17.009 & (Dominated) $^{a}$ & $\$ 4,549.99$ & 17.009 & (Dominated) $^{a}$ \\
\hline $\mathrm{FOBT}+\mathrm{COX}-2$ & $\$ 62,388.23$ & 17.089 & (Dominated) $^{a}$ & $\$ 62,388.23$ & 17.089 & (Dominated $^{a}$ & $\$ 62,388.23$ & 17.089 & (Dominated) $^{2}$ \\
\hline Colonoscopy + COX-2 & $\$ 62,810.35$ & 17.207 & (Dominated) $^{a}$ & $\$ 62,810.35$ & 17.207 & (Dominated) $^{a}$ & $\$ 62,810.35$ & 17.207 & 80571240.87 \\
\hline $\mathrm{FOBT} / \mathrm{FS}+\mathrm{COX}-2$ & $\$ 64,210.65$ & 17.002 & (Dominated) $^{a}$ & $\$ 64,210.65$ & 17.002 & (Dominated) $^{a}$ & $\$ 64,210.65$ & 17.002 & 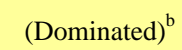 \\
\hline
\end{tabular}

FOBT = annual Fecal Occult Blood Test

FOBT/FS - annual Fecal Occult Blood Test and Flexible Sigmoidoscopy every five years

Colonoscopy - Colonoscopy every ten years

ASA - aspirin, 81 milligrams daily

COX-2 - Cyclooxygenase-2 Inhibitor, 425 milligrams daily

ICER = Incremental Cost-Effectiveness Ratio

aDominated by Colonoscopy plus ASA

${ }^{b}$ Dominated by Colonoscopy plus COX-2 
Table 4-17: One Way Sensitivity Analyses Results of Colorectal Cancer Screening Strategies and Chemopreventive Agents Parameter: pPolyp_Well_Colon_COX2

\begin{tabular}{|c|c|c|c|c|c|c|c|c|c|}
\hline & Base Case: & 0.123336 & & Lower Range: & 0.114771 & & Upper Range: & 0.131901 & \\
\hline Screening Strategy & Cost & Effectiveness & ICER & Cost & Effectiveness & ICER & Cost & Effectiveness & ICER \\
\hline FOBT & $\$ 2,295.90$ & 17.041 & $\$ 13,014.95$ & $\$ 2,295.90$ & 17.041 & $\$ 13,014.95$ & $\$ 2,295.90$ & 17.041 & $\$ 13,014.95$ \\
\hline $\mathrm{FOBT}+\mathrm{ASA}$ & $\$ 2,673.96$ & 17.094 & $\$ 7,173.55$ & $\$ 2,673.96$ & 17.094 & $\$ 7,173.55$ & $\$ 2,673.96$ & 17.094 & $\$ 7,173.55$ \\
\hline Colonoscopy & $\$ 2,677.60$ & 17.196 & $\$ 35.43$ & $\$ 2,677.60$ & 17.196 & $\$ 35.43$ & $\$ 2,677.60$ & 17.196 & $\$ 35.43$ \\
\hline Colonoscopy + ASA & $\$ 3,003.21$ & 17.208 & $\$ 27,513.64$ & $\$ 3,003.21$ & 17.208 & $\$ 27,513.64$ & $\$ 3,003.21$ & 17.208 & $\$ 27,513.64$ \\
\hline FOBT/FS & $\$ 3,991.03$ & 16.932 & $(\text { Dominated })^{a}$ & $\$ 3,991.03$ & 16.932 & $(\text { Dominated })^{a}$ & $\$ 3,991.03$ & 16.932 & $(\text { Dominated })^{a}$ \\
\hline $\mathrm{FOBT} / \mathrm{FS}+\mathrm{ASA}$ & $\$ 4,549.99$ & 17.009 & $(\text { Dominated })^{a}$ & $\$ 4,549.99$ & 17.009 & $(\text { Dominated })^{a}$ & $\$ 4,549.99$ & 17.009 & $(\text { Dominated })^{a}$ \\
\hline $\mathrm{FOBT}+\mathrm{COX}-2$ & $\$ 62,388.23$ & 17.089 & $(\text { Dominated })^{a}$ & $\$ 62,388.23$ & 17.089 & $(\text { Dominated })^{a}$ & $\$ 62,388.23$ & 17.089 & (Dominated) $^{a}$ \\
\hline Colonoscopy + COX-2 & $\$ 62,810.35$ & 17.207 & (Dominated) $^{a}$ & $\$ 62,811.60$ & 17.209 & 80797686.42 & $\$ 62,809.10$ & 17.205 & (Dominated) $^{a}$ \\
\hline FOBT/FS + COX-2 & $\$ 64,210.65$ & 17.002 & ${\text { (Dominated })^{a}}^{a}$ & $\$ 64,210.65$ & 17.002 & (Dominated) $^{\mathrm{b}}$ & $\$ 64,210.65$ & 17.002 & (Dominated) $^{a}$ \\
\hline
\end{tabular}

FOBT = annual Fecal Occult Blood Test

FOBT/FS - annual Fecal Occult Blood Test and Flexible Sigmoidoscopy every five years

Colonoscopy - Colonoscopy every ten years

ASA - aspirin, 81 milligrams daily

COX-2 - Cyclooxygenase-2 Inhibitor, 425 milligrams daily

ICER = Incremental Cost-Effectiveness Ratio

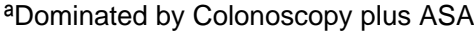

${ }^{\mathrm{b}}$ Dominated by Colonoscopy plus COX-2 
Table 4-18: One Way Sensitivity Analyses Results of Colorectal Cancer Screening Strategies and Chemopreventive Agents Parameter: pCancer_Well_FOBT_ASA

\begin{tabular}{|c|c|c|c|c|c|c|c|c|c|}
\hline & Base Case: & 0.00945 & & Lower Range: & 0.008775 & & Upper Range: & 0.010125 & \\
\hline Screening Strategy & Cost & Effectiveness & ICER & Cost & Effectiveness & ICER & Cost & Effectiveness & ICER \\
\hline FOBT & $\$ 2,295.90$ & 17.041 & $\$ 13,014.95$ & $\$ 2,295.90$ & 17.041 & $\$ 13,014.95$ & $\$ 2,295.90$ & 17.041 & $\$ 13,014.95$ \\
\hline FOBT + aspirin & $\$ 2,673.96$ & 17.094 & $\$ 7,173.55$ & $\$ 2,671.07$ & 17.096 & $\$ 6,812.88$ & $\$ 2,676.83$ & 17.091 & $\$ 7,173.55$ \\
\hline Colonoscopy & $\$ 2,677.60$ & 17.196 & $\$ 35.43$ & $\$ 2,677.60$ & 17.196 & $\$ 64.91$ & $\$ 2,677.60$ & 17.196 & $\$ 35.43$ \\
\hline Colonoscopy + aspirin & $\$ 3,003.21$ & 17.208 & $\$ 27,513.64$ & $\$ 3,003.21$ & 17.208 & $\$ 27,513.64$ & $\$ 3,003.21$ & 17.208 & $\$ 27,513.64$ \\
\hline FOBT/FlexSig & $\$ 3,991.03$ & 16.932 & ${\text { (Dominated })^{a}}^{a}$ & $\$ 3,991.03$ & 16.932 & 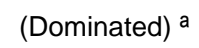 & $\$ 3,991.03$ & 16.932 & ${\text { (Dominated })^{a}}^{a}$ \\
\hline FOBT/FlexSig + aspirin & $\$ 4,549.99$ & 17.009 & (Dominated) $^{a}$ & $\$ 4,548.17$ & 17.011 & (Dominated) $^{a}$ & $\$ 4,551.81$ & 17.007 & (Dominated) $^{a}$ \\
\hline $\mathrm{FOBT}+\mathrm{COX}-2$ & $\$ 62,388.23$ & 17.089 & (Dominated) $^{a}$ & $\$ 62,388.23$ & 17.089 & 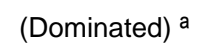 & $\$ 62,388.23$ & 17.089 & (Dominated $^{a}$ \\
\hline Colonoscopy + COX-2 & $\$ 62,810.35$ & 17.207 & (Dominated) $^{a}$ & $\$ 62,810.35$ & 17.207 & (Dominated) $^{a}$ & $\$ 62,810.35$ & 17.207 & (Dominated) $^{a}$ \\
\hline FOBT/FlexSig + COX-2 & $\$ 64,210.65$ & 17.002 & (Dominated) $^{a}$ & $\$ 64,210.65$ & 17.002 & (Dominated) $^{a}$ & $\$ 64,210.65$ & 17.002 & (Dominated) $^{a}$ \\
\hline
\end{tabular}

FOBT = annual Fecal Occult Blood Test

FOBT/FS - annual Fecal Occult Blood Test and Flexible Sigmoidoscopy every five years

Colonoscopy - Colonoscopy every ten years

ASA - aspirin, 81 milligrams daily

COX-2 - Cyclooxygenase-2 Inhibitor, 425 milligrams daily

ICER = Incremental Cost-Effectiveness Ratio

aDominated by Colonoscopy plus ASA 
Table 4-19: One Way Sensitivity Analyses Results of Colorectal Cancer Screening Strategies and Chemopreventive Agents Parameter: pCancer_Well_FOBT_COX2

\begin{tabular}{|c|c|c|c|c|c|c|c|c|c|}
\hline & Base Case: & 0.00972 & & Lower Range: & 0.009045 & & Upper Range: & 0.010395 & \\
\hline Screening Strategy & Cost & Effectiveness & ICER & Cost & Effectiveness & ICER & Cost & Effectiveness & ICER \\
\hline FOBT & $\$ 2,295.90$ & 17.041 & $\$ 13,014.95$ & $\$ 2,295.90$ & 17.041 & $\$ 13,014.95$ & $\$ 2,295.90$ & 17.041 & $\$ 13,014.95$ \\
\hline $\mathrm{FOBT}+\mathrm{ASA}$ & $\$ 2,673.96$ & 17.094 & $\$ 7,173.55$ & $\$ 2,673.96$ & 17.094 & $\$ 7,173.55$ & $\$ 2,673.96$ & 17.094 & $\$ 7,173.55$ \\
\hline Colonoscopy & $\$ 2,677.60$ & 17.196 & $\$ 35.43$ & $\$ 2,677.60$ & 17.196 & $\$ 35.43$ & $\$ 2,677.60$ & 17.196 & $\$ 35.43$ \\
\hline Colonoscopy + ASA & $\$ 3,003.21$ & 17.208 & $\$ 27,513.64$ & $\$ 3,003.21$ & 17.208 & $\$ 27,513.64$ & $\$ 3,003.21$ & 17.208 & $\$ 27,513.64$ \\
\hline FOBT/FS & $\$ 3,991.03$ & 16.932 & ${\text { (Dominated })^{a}}^{a}$ & $\$ 3,991.03$ & 16.932 & ${\text { (Dominated })^{a}}^{a}$ & $\$ 3,991.03$ & 16.932 & ${\text { (Dominated })^{a}}^{a}$ \\
\hline $\mathrm{FOBT} / \mathrm{FS}+\mathrm{ASA}$ & $\$ 4,549.99$ & 17.009 & (Dominated) $^{a}$ & $\$ 4,549.99$ & 17.009 & (Dominated) $^{a}$ & $\$ 4,549.99$ & 17.009 & ${\text { (Dominated })^{a}}^{a}$ \\
\hline $\mathrm{FOBT}+\mathrm{COX}-2$ & $\$ 62,388.23$ & 17.089 & (Dominated) $^{a}$ & $\$ 62,392.27$ & 17.092 & (Dominated $^{a}$ & $\$ 62,384.18$ & 17.087 & (Dominated $^{a}$ \\
\hline Colonoscopy + COX-2 & $\$ 62,810.35$ & 17.207 & (Dominated) $^{a}$ & $\$ 62,810.35$ & 17.207 & (Dominated) $^{a}$ & $\$ 62,810.35$ & 17.207 & (Dominated) $^{a}$ \\
\hline FOBT/FS + COX-2 & $\$ 64,210.65$ & 17.002 & (Dominated) $^{a}$ & $\$ 64,215.15$ & 17.004 & (Dominated) $^{a}$ & $\$ 64,206.15$ & 17 & (Dominated) $^{a}$ \\
\hline
\end{tabular}

FOBT = annual Fecal Occult Blood Test

FOBT/FS - annual Fecal Occult Blood Test and Flexible Sigmoidoscopy every five years

Colonoscopy - Colonoscopy every ten years

ASA - aspirin, 81 milligrams daily

COX-2 - Cyclooxygenase-2 Inhibitor, 425 milligrams daily

ICER = Incremental Cost-Effectiveness Ratio

aDominated by Colonoscopy plus ASA 
Table 4-20: One Way Sensitivity Analyses Results of Colorectal Cancer Screening Strategies and Chemopreventive Agents Parameter: pCancer_Well_FOBTFS_ASA

\begin{tabular}{|c|c|c|c|c|c|c|c|c|c|}
\hline & Base Case: & 0.00672 & & Lower Range: & 0.006214 & & Upper Range: & 0.00717 & \\
\hline Screening Strategy & Cost & Effectiveness & ICER & Cost & Effectiveness & ICER & Cost & Effectiveness & ICER \\
\hline FOBT & $\$ 2,295.90$ & 17.041 & $\$ 13,014.95$ & $\$ 2,295.90$ & 17.041 & $\$ 13,014.95$ & $\$ 2,295.90$ & 17.041 & $\$ 13,014.95$ \\
\hline $\mathrm{FOBT}+\mathrm{ASA}$ & $\$ 2,673.96$ & 17.094 & $\$ 7,173.55$ & $\$ 2,673.96$ & 17.094 & $\$ 7,173.55$ & $\$ 2,673.96$ & 17.094 & $\$ 7,173.55$ \\
\hline Colonoscopy & $\$ 2,677.60$ & 17.196 & $\$ 35.43$ & $\$ 2,677.60$ & 17.196 & $\$ 35.43$ & $\$ 2,677.60$ & 17.196 & $\$ 35.43$ \\
\hline Colonoscopy + ASA & $\$ 3,003.21$ & 17.208 & $\$ 27,513.64$ & $\$ 3,003.21$ & 17.208 & $\$ 27,513.64$ & $\$ 3,003.21$ & 17.208 & $\$ 27,513.64$ \\
\hline FOBT/FS & $\$ 3,991.03$ & 16.932 & ${\text { (Dominated })^{a}}^{a}$ & $\$ 3,991.03$ & 16.932 & (Dominated) $^{a}$ & $\$ 3,991.03$ & 16.932 & ${\text { (Dominated })^{a}}^{a}$ \\
\hline $\mathrm{FOBT} / \mathrm{FS}+\mathrm{ASA}$ & $\$ 4,549.94$ & 17.009 & ${\text { (Dominated })^{a}}^{a}$ & $\$ 4,548.99$ & 17.01 & $(\text { Dominated })^{a}$ & $\$ 4,550.89$ & 17.008 & $(\text { Dominated })^{a}$ \\
\hline $\mathrm{FOBT}+\mathrm{COX}-2$ & $\$ 62,388.23$ & 17.089 & (Dominated) $^{a}$ & $\$ 62,388.23$ & 17.089 & (Dominated) $^{a}$ & $\$ 62,388.23$ & 17.089 & ${\text { (Dominated })^{a}}^{a}$ \\
\hline Colonoscopy + COX-2 & $\$ 62,810.35$ & 17.207 & (Dominated) $^{a}$ & $\$ 62,810.35$ & 17.207 & (Dominated) $^{a}$ & $\$ 62,810.35$ & 17.207 & (Dominated) $^{a}$ \\
\hline $\mathrm{FOBT} / \mathrm{FS}+\mathrm{COX}-2$ & $\$ 64,210.65$ & 17.002 & (Dominated) $^{a}$ & $\$ 64,210.65$ & 17.002 & (Dominated) $^{a}$ & $\$ 64,210.65$ & 17.002 & ${\text { (Dominated })^{a}}^{a}$ \\
\hline
\end{tabular}

FOBT = annual Fecal Occult Blood Test

FOBT/FS - annual Fecal Occult Blood Test and Flexible Sigmoidoscopy every five years

Colonoscopy - Colonoscopy every ten years

ASA - aspirin, 81 milligrams daily

COX-2 - Cyclooxygenase-2 Inhibitor, 425 milligrams daily

ICER = Incremental Cost-Effectiveness Ratio

aDominated by Colonoscopy plus ASA 
Table 4-21: One Way Sensitivity Analyses Results of Colorectal Cancer Screening Strategies and Chemopreventive Agents Parameter: pCancer_Well_FOBTFS_COX2

\begin{tabular}{|c|c|c|c|c|c|c|c|c|c|}
\hline & Base Case: & 0.006912 & & Lower Range: & 0.0064052 & & Upper Range: & 0.0073612 & \\
\hline Screening Strategy & Cost & Effectiveness & ICER & Cost & Effectiveness & ICER & Cost & Effectiveness & ICER \\
\hline FOBT & $\$ 2,295.90$ & 17.041 & $\$ 13,014.95$ & $\$ 2,295.90$ & 17.041 & $\$ 13,014.95$ & $\$ 2,295.90$ & 17.041 & $\$ 13,014.95$ \\
\hline $\mathrm{FOBT}+\mathrm{ASA}$ & $\$ 2,673.96$ & 17.094 & $\$ 7,173.55$ & $\$ 2,673.96$ & 17.094 & $\$ 7,173.55$ & $\$ 2,673.96$ & 17.094 & $\$ 7,173.55$ \\
\hline Colonoscopy & $\$ 2,677.60$ & 17.196 & $\$ 35.43$ & $\$ 2,677.60$ & 17.196 & $\$ 35.43$ & $\$ 2,677.60$ & 17.196 & $\$ 35.43$ \\
\hline Colonoscopy + ASA & $\$ 3,003.21$ & 17.208 & $\$ 27,513.64$ & $\$ 3,003.21$ & 17.208 & $\$ 27,513.64$ & $\$ 3,003.21$ & 17.208 & $\$ 27,513.64$ \\
\hline FOBT/FS & $\$ 3,991.03$ & 16.932 & ${\text { (Dominated })^{a}}^{a}$ & $\$ 3,991.03$ & 16.932 & ${\text { (Dominated })^{a}}^{a}$ & $\$ 3,991.03$ & 16.932 & ${\text { (Dominated })^{a}}^{a}$ \\
\hline $\mathrm{FOBT} / \mathrm{FS}+\mathrm{ASA}$ & $\$ 4,549.99$ & 17.009 & (Dominated) $^{a}$ & $\$ 4,549.99$ & 17.009 & (Dominated) $^{a}$ & $\$ 4,549.99$ & 17.009 & $\left(\right.$ Dominated) ${ }^{a}$ \\
\hline $\mathrm{FOBT}+\mathrm{COX}-2$ & $\$ 62,388.23$ & 17.089 & (Dominated) $^{a}$ & $\$ 62,388.23$ & 17.089 & (Dominated $^{a}$ & $\$ 62,388.23$ & 17.089 & (Dominated $^{a}$ \\
\hline Colonoscopy + COX-2 & $\$ 62,810.35$ & 17.207 & (Dominated) $^{a}$ & $\$ 62,810.35$ & 17.207 & (Dominated) $^{a}$ & $\$ 62,810.35$ & 17.207 & (Dominated) $^{a}$ \\
\hline FOBT/FS + COX-2 & $\$ 64,210.79$ & 17.002 & (Dominated) $^{a}$ & $\$ 64,213.20$ & 17.004 & (Dominated) $^{a}$ & $\$ 64,208.39$ & 17.001 & (Dominated) $^{a}$ \\
\hline
\end{tabular}

FOBT = annual Fecal Occult Blood Test

FOBT/FS - annual Fecal Occult Blood Test and Flexible Sigmoidoscopy every five years

Colonoscopy - Colonoscopy every ten years

ASA - aspirin, 81 milligrams daily

COX-2 - Cyclooxygenase-2 Inhibitor, 425 milligrams daily

ICER = Incremental Cost-Effectiveness Ratio

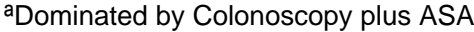


Table 4-22: One Way Sensitivity Analyses Results of Colorectal Cancer Screening Strategies and Chemopreventive Agents Parameter: pCancer_Well_Colon_ASA

\begin{tabular}{|c|c|c|c|c|c|c|c|c|c|}
\hline & Base Case: & 0.00336 & & Lower Range: & 0.00312 & & Upper Range: & 0.0036 & \\
\hline Screening Strategy & Cost & Effectiveness & ICER & Cost & Effectiveness & ICER & Cost & Effectiveness & ICER \\
\hline FOBT & $\$ 2,295.90$ & 17.041 & $\$ 13,014.95$ & $\$ 2,295.90$ & 17.041 & $\$ 13,014.95$ & $\$ 2,295.90$ & 17.041 & $\$ 13,014.95$ \\
\hline $\mathrm{FOBT}+\mathrm{ASA}$ & $\$ 2,673.96$ & 17.094 & $\$ 7,173.55$ & $\$ 2,673.96$ & 17.094 & $\$ 7,173.55$ & $\$ 2,673.96$ & 17.094 & $\$ 7,173.55$ \\
\hline Colonoscopy & $\$ 2,677.60$ & 17.196 & $\$ 35.43$ & $\$ 2,677.60$ & 17.196 & $\$ 35.43$ & $\$ 2,677.60$ & 17.196 & $\$ 35.43$ \\
\hline Colonoscopy + ASA & $\$ 3,003.21$ & 17.208 & $\$ 27,513.64$ & $\$ 3,002.83$ & 17.209 & $\$ 26,869.97$ & $\$ 3,003.59$ & 17.208 & $\$ 28,187.27$ \\
\hline FOBT/FS & $\$ 3,991.03$ & 16.932 & ${\text { (Dominated })^{a}}^{a}$ & $\$ 3,991.03$ & 16.932 & ${\text { (Dominated })^{a}}^{a}$ & $\$ 3,991.03$ & 16.932 & ${\text { (Dominated })^{a}}^{a}$ \\
\hline $\mathrm{FOBT} / \mathrm{FS}+\mathrm{ASA}$ & $\$ 4,549.99$ & 17.009 & (Dominated) $^{a}$ & $\$ 4,549.99$ & 17.009 & (Dominated) $^{a}$ & $\$ 4,549.99$ & 17.009 & ${\text { (Dominated })^{a}}^{a}$ \\
\hline $\mathrm{FOBT}+\mathrm{COX}-2$ & $\$ 62,388.23$ & 17.089 & (Dominated) $^{a}$ & $\$ 62,388.23$ & 17.089 & (Dominated $^{a}$ & $\$ 62,388.23$ & 17.089 & (Dominated $^{a}$ \\
\hline Colonoscopy + COX-2 & $\$ 62,810.35$ & 17.207 & (Dominated) $^{a}$ & $\$ 62,810.35$ & 17.207 & (Dominated) $^{a}$ & $\$ 62,810.35$ & 17.207 & (Dominated) $^{a}$ \\
\hline FOBT/FS + COX-2 & $\$ 64,210.65$ & 17.002 & (Dominated) $^{a}$ & $\$ 64,210.65$ & 17.002 & (Dominated) $^{a}$ & $\$ 64,210.65$ & 17.002 & (Dominated) $^{a}$ \\
\hline
\end{tabular}

FOBT = annual Fecal Occult Blood Test

FOBT/FS - annual Fecal Occult Blood Test and Flexible Sigmoidoscopy every five years

Colonoscopy - Colonoscopy every ten years

ASA - aspirin, 81 milligrams daily

COX-2 - Cyclooxygenase-2 Inhibitor, 425 milligrams daily

ICER = Incremental Cost-Effectiveness Ratio

aDominated by Colonoscopy plus ASA 
Table 4-23: One Way Sensitivity Analyses Results of Colorectal Cancer Screening Strategies and Chemopreventive Agents Parameter: pCancer_Well_Colon_COX2

\begin{tabular}{|c|c|c|c|c|c|c|c|c|c|}
\hline & Base Case: & 0.003456 & & Lower Range: & 0.003216 & & Upper Range: & 0.003696 & \\
\hline Screening Strategy & Cost & Effectiveness & ICER & Cost & Effectiveness & ICER & Cost & Effectiveness & ICER \\
\hline FOBT & $\$ 2,295.90$ & 17.041 & $\$ 13,014.95$ & $\$ 2,295.90$ & 17.041 & $\$ 13,014.95$ & $\$ 2,295.90$ & 17.041 & $\$ 13,014.95$ \\
\hline $\mathrm{FOBT}+\mathrm{ASA}$ & $\$ 2,673.96$ & 17.094 & $\$ 7,173.55$ & $\$ 2,673.96$ & 17.094 & $\$ 7,173.55$ & $\$ 2,673.96$ & 17.094 & $\$ 7,173.55$ \\
\hline Colonoscopy & $\$ 2,677.60$ & 17.196 & $\$ 35.43$ & $\$ 2,677.60$ & 17.196 & $\$ 35.43$ & $\$ 2,677.60$ & 17.196 & $\$ 35.43$ \\
\hline Colonoscopy + ASA & $\$ 3,003.21$ & 17.208 & $\$ 27,513.64$ & $\$ 3,003.21$ & 17.208 & $\$ 27,513.64$ & $\$ 3,003.21$ & 17.208 & $\$ 27,513.64$ \\
\hline FOBT/FS & $\$ 3,991.03$ & 16.932 & ${\text { (Dominated })^{a}}^{a}$ & $\$ 3,991.03$ & 16.932 & ${\text { (Dominated })^{a}}^{a}$ & $\$ 3,991.03$ & 16.932 & ${\text { (Dominated })^{a}}^{a}$ \\
\hline $\mathrm{FOBT} / \mathrm{FS}+\mathrm{ASA}$ & $\$ 4,549.99$ & 17.009 & (Dominated) $^{a}$ & $\$ 4,549.99$ & 17.009 & (Dominated) $^{a}$ & $\$ 4,549.99$ & 17.009 & $\left(\right.$ Dominated) ${ }^{a}$ \\
\hline $\mathrm{FOBT}+\mathrm{COX}-2$ & $\$ 62,388.23$ & 17.089 & (Dominated) $^{a}$ & $\$ 62,388.23$ & 17.089 & (Dominated $^{a}$ & $\$ 62,388.23$ & 17.089 & (Dominated $^{a}$ \\
\hline Colonoscopy + COX-2 & $\$ 62,810.35$ & 17.207 & (Dominated) $^{a}$ & $\$ 62,810.76$ & 17.208 & (Dominated) $^{a}$ & $\$ 62,809.95$ & 17.207 & (Dominated) $^{a}$ \\
\hline FOBT/FS + COX-2 & $\$ 64,210.65$ & 17.002 & (Dominated) $^{a}$ & $\$ 64,210.65$ & 17.002 & (Dominated) $^{a}$ & $\$ 64,210.65$ & 17.002 & (Dominated) $^{a}$ \\
\hline
\end{tabular}

FOBT = annual Fecal Occult Blood Test

FOBT/FS - annual Fecal Occult Blood Test and Flexible Sigmoidoscopy every five years

Colonoscopy - Colonoscopy every ten years

ASA - aspirin, 81 milligrams daily

COX-2 - Cyclooxygenase-2 Inhibitor, 425 milligrams daily

ICER = Incremental Cost-Effectiveness Ratio

aDominated by Colonoscopy plus ASA 


\section{Discussion and Conclusions}

The primary goal of this study was to conduct a cost-effectiveness evaluation of colorectal cancer (CRC) screening procedures, as well as to determine the usefulness of aspirin and a cyclooxygenase-2 (COX-2) inhibitor as chemopreventive agents. Since recommendations for CRC screening vary among professional organizations, and opinions regarding the screening procedures are even more diverse, the determination of the most cost-effective screening strategy aids in decision making for both patients and physicians. This decision involves choosing the strategy that is most effective, least costly, and best suited for the individual patient. The detailed methodology for determination of cost-effectiveness of the alternative CRC screening strategies is reported in Chapter 3. The results of the decision model are presented in Chapter 4. This chapter discusses the major study findings and their significance. It also includes the major limitations of the study and presents the significance of the study results. Finally, some recommendations for future research are also included in this chapter.

\subsection{Review of Findings}

Cost-effectiveness analysis is an economic evaluation method that can be used to compare currently recommended screening strategies to aid in informed decision-making. The incremental cost-effectiveness ratio (ICER) was calculated as the additional cost of a screening strategy compared to that of the next most cost-effective strategy. The decision model was developed to determine the costs, effectiveness and ICER of currently recommended CRC screening strategies in the presence and absence of chemopreventive agents. The cost-effectiveness analysis was conducted from the societal perspective. 
Results from the analysis showed that the most cost-effective screening strategy was the use of a fecal occult blood test (FOBT). In terms of only cost, FOBT is the lowest screening strategy whereas the highest is colonoscopy plus COX-2 inhibitor. This strategy incurs the highest cost mainly due to the cost of COX-2 (\$3,390.24 per year). In addition, colonoscopy has the highest cost among the three screening procedures. The most effective strategy is colonoscopy plus aspirin, and FOBT/Flexible Sigmoidoscopy (FS) was found to be least effective. Colonoscopy plus aspirin has the highest effectiveness because colonoscopy has the highest effectiveness among the three screening procedures, as well as the fact that aspirin and COX-2 have an addition benefit of effectives to the screening procedures. The effectiveness of colonoscopy plus aspirin (17.2083) and colonoscopy plus COX-2 (17.2072) was very close.

One-way sensitivity analysis was conducted to identify key variables that may have an impact on the ICERs of the screening strategies. The ICER of the strategies were sensitive to the costs of FOBT and colonoscopy, as well as the cost of polypectomy. If the cost of FOBT would be raised slightly (\$0.64), or the cost of colonoscopy reduced slightly (\$3.80), the ICER would be affected so that the strategy FOBT plus aspirin would become dominated by colonoscopy. This domination occurs because the cost of colonoscopy now becomes lower than the cost of FOBT plus aspirin, while the effectiveness remains higher. Additionally, if the cost of polypectomy increased by approximately $\$ 30.00$, FOBT plus aspirin would again become dominated by colonoscopy. Again, this means the cost of colonoscopy now becomes lower than the cost of FOBT plus aspirin, while the effectiveness remains higher. The ICER of FOBT plus aspirin and colonoscopy were also quite sensitive to the cost of aspirin. Aspirin has 
a base case cost of $\$ 18.00$ per year, and if this cost were increased to $\$ 18.21$, FOBT plus aspirin would become dominated by colonoscopy yet again. However, the ICERs were not sensitive to the cost of COX-2 inhibitor.

This study is among the first to employ a decision analysis model to evaluate the most commonly utilized screening procedures (FOBT, FS, and colonoscopy), along with the addition of aspirin and COX-2 inhibitor as chemopreventive agents. Four previous studies have evaluated the cost-effectiveness of the three screening procedures used in this study (Wagner and colleagues, 1996,; Frazier and colleagues, 2000; Khandker and colleagues, 2000; Vijan and colleagues, 2001). Each of these studies measured outcomes in cost per life years saved. This study uses the measure of quality adjusted life years (QALYs). Vijan and colleagues (Vijan, et al., 2001) concluded that FOBT was the most cost-effective screening strategies, followed by colonoscopy, FS, and lastly FOBT/FS. The results of the Wagner study (Wagner, et al., 1996) were similar, except colonoscopy was more cost-effective than FOBT. Frazer (Frazier, et al., 2000) and Khandker (Khandker, at al., 2000) both concluded that FS was the most cost-effective, followed by FOBT. However, the difference between these two studies is that colonoscopy was more cost-effective than FOBT/FS in the Frazier study, while Khandker found the combination strategy to be more cost-effective than colonoscopy.

The screening strategies of FOBT, FOBT plus aspirin, colonoscopy, and colonoscopy plus aspirin all have an ICER of less than $\$ 50,000$ per QALY. In health economics, a figure of $\$ 50,000$ per QALY is often suggested as the upper limit of an acceptable ICER. No previous decision analysis study has evaluated both aspirin and COX-2 inhibitor as chemopreventive agents. 


\subsection{Implications of Study Findings}

The study results will be a valuable addition to the scientific literature in the field of CRC screening. In addition to its academic importance, the study results have implications for decision makers of CRC screening; physicians, administrators, and patients.

The results from the study suggest that FOBT, FOBT plus aspirin, colonoscopy, and colonoscopy plus aspirin are the more cost-effective of all the screening strategies employed. FOBT plus aspirin and colonoscopy have similar cost-effectiveness with colonoscopy having an ICER of only $\$ 35.43$. This is due to both strategies being quite sensitive to the cost of FOBT and colonoscopy.

\subsection{Study Limitations}

This study has limitations which are noted below. These limitations need to be considered when deriving inferences from the reported results.

1. A decision model for economic evaluations is only as good as the data that is used to populate the model. The impact of uncertainty in the parameter estimates on the results was evaluated by conducting a sensitivity analysis.

2. The base case analysis assumes that the study population used for parameter estimates are comparable in their demographic and clinical characteristics. In reality, this is possibly only through randomization of the cohort to each screening strategy included in the model. 
3. In this study, we assumed $100 \%$ compliance for screening. Vijan and colleagues (2001) report that colonoscopy at ages 50 and 60 years is the preferred test regardless of compliance with the primary screening test. However, if follow-up colonoscopy for polyps is less than $75 \%$, then even once-lifetime colonoscopy is preferred over most combinations of flexible sigmoidoscopy (FS) and FOBT. This may suggest that compliance regarding recommended screening intervals does not directly affect the effectiveness of the screening strategy.

4. In this model, the treatment rate was set at $100 \%$. That is, everyone who is diagnosed with CRC would be treated. Although this is not true in clinical practice, a specific rate could not be otherwise found to estimate this. Therefore, the $100 \%$ rate was used to estimate treatment.

5. The screening procedures of FOBT and FS were modeled together as a combined strategy. The strategy was modeled after the study by Winawer and colleagues (1997). This strategy involves an annual FOBT with a FS every five years. The fifth year screening estimate is not a combined probability of FOBT and FS. If on the fifth year the FOBT returns a positive test, the FS is foregone and a diagnostic colonoscopy is performs. 


\subsection{Recommendations for Future Research}

As mentioned previously, an economic model is only as good as the data used to develop it. The model should be populated with estimates of effectiveness and costs of each screening procedure and chemopreventive agents from their use in clinical practice. Future research should be targeted at developing a decision model that incorporates compliance with screening procedures. Additionally, the probability of treatment should be taken into consideration in the model.

This study incorporated screening procedures which are most common in current practice. As newer technologies emerge, and screening guidelines are revised, future studies should incorporate these factors to assess their cost-effectiveness.

\subsection{Conclusions}

In this study, fecal occult blood test (FOBT) and colonoscopy were the most costeffective screening procedures in this study. The strategies that incorporated these procedures, along with the addition of aspirin, were the four dominant strategies in this study. The combination strategies of FOBT/FS was the least cost-effective of the procedures, regardless of the addition of chemopreventive agents. Strategies that incorporated a Cox-2 inhibitor as the chemopreventive agent were the least cost-effective due to the prohibitive cost of the drug. It did, however, prove to increase the effectiveness of screening procedures. If the cost of Cox-2 inhibitors were lower, these screening strategies would become more competitive with those that incorporate aspirin as the chemopreventive agent. However, until then, aspirin remains as the most costeffective chemopreventive addition to CRC screening procedures. 


\section{$\underline{\text { BIBLIOGRAPHY }}$}

American Cancer Society (2001). Cancer facts and figures. Atlanta, Georgia: American Cancer Society.

American Cancer Society (2005). Cancer facts and figures. Atlanta, Georgia: American Cancer Society.

American Gastroenterological Association, Foundation for Digestive Health and Nutrition (2002).

Arguedas, M.R., Heudebert, G.R. \& Wilcox, C.M. (2001). Surveillance colonoscopy or chemoprevention with COX-2 inhibitors in average-risk post-polypectomy patients: a decision analysis. Aliment Pharmacol Ther, 15, 631-638.

Autier, P., Boyle, P., Buyse, M. \& Bleiberg, H. (2003). Is FOBT screening really the answer for lowering mortality in colorectal cancer? Recent Results Cancer Res, 163, 254-263.

Baron, J.A., Cole, B.F., Sandler, R.S., et. al. (2003). A randomized trial of aspirin to prevent colorectal adenomas. N Engl J Med, 348(10), 891-899.

Baron, J.A., Sandler, R.S. (2000). Nonsteroidal anti-inflammatory drugs and cancer prevention. Annu Rev Med, 51, 511-523.

Batelle Corporation. (1998). Evaluation of physicians' knowledge, attitudes, and practices related to screening for colorectal cancer. Atlanta, GA: American Cancer Society.

Bero, L.A., Grilli, R., Grimshaw, J.M., Harvey, E., Osman, A.D., Thomson, M.A., et al. (1998). Closing the gap between research and practice: an overview of systematic reviews of interventions to promote the implementation of research findings. Brit Med Journal, 317, 465-468.

Byers, T., Levin, B., Rothenberger, D., Dodd, G.D. \& Smith, R.A. (1997). American Cancer Society guidelines for screening and surveillance for early detection of colorectal polyps and cancer: Update 1997. American Cancer Society Detection and Treatment Advisory Group on Colorectal Cancer. CA Cancer J Clin, 47(3), 154-160.

Caldwell, B., Aldington, S., Weatherall, M., Shirtcliffe, P., Beasley, R. (2006). Risk of cardiovascular events and celecoxib: a systematic review and meta-analysis. $J$ 
R Soc Med, 99(3), 132-40.

Cancer Research and Prevention Foundation (2004). Alexandria, Va.

Centers for Disease Control and Prevention (2002). National Center for Chronic Disease Prevention and Health Promotion. The Burden of Chronic Diseases and Their Risk Factors. National and State Perspectives 2002. February 2002.

Centers for Disease Control and Prevention (2002). Recent Trends in Mortality Rates for Four Major Cancers, by Sex and Race/Ethnicity - United States, 1990-1998. Morb Mortal Wkly Rep, 51(03), 49-53.

Centers for Medicare \& Medicaid Services, (2001). Baltimore, Maryland. Transmittal AB 01-81. May 15, 2001.

Chan, A.T., Giovannucci, E.L., Meyerhardt, J.A., Schernhammer, E.S., Curhan, G.C. \& Fuchs, C.S. (2005). Long-term use of aspirin and nonsteroidal anti-inflammatory drugs and risk of colorectal cancer. JAMA, 294(8), 914-23.

Colon Cancer Alliance. (2001). New York, NY.

Cooper, GS., Fortinsky, R.H., Hapke, R. \& Landefeld, C.S. (1998). Factors associated with the use of flexible sigmoidoscopy as a screening test for the detection of colorectal carcinoma by primary care physicians. Cancer, 82, 1476-1481.

Daniels, K. \& McKee, M. (1995). Options for screening for colorectal cancer in the Royal Air Force: a cost-effectiveness evaluation. J R Army Med Corps, 141(3), $142-50$.

DuBois, R.B., Giardiello, F.M. \& Smalley, W.E. (1996). Nonsteroidal anti inflammatory drugs, eicosanoids, and colorectal cancer prevention. Gastroenterol Clin North Am, 25, 773-791.

Frazier, A.L., Colditz, G.A., Fuchs, C.S. \& Kuntz, K.M. (2000). Cost-effectiveness of screening for colorectal cancer in the general population. JAMA, 284, 1954-1961.

Glick, S.N., Ralls, P.W., Balfe, D.M., Bree, R.L., DiSantis, D.J., Kidd, R., et al. (2000). Screening for colorectal cancer. American College of Radiology. ACR Appropriateness Criteria. Radiology, 215 Suppl, 231-237.

Greenberg, E.R., Baron, J.A., Freeman, D.H., Mandel, J.S \&, Haile, R. (1993). Reduced risk of large bowel adenomas among aspirin users. The Polyp 
Prevention Study Group. J Natl Cancer Inst, 85(11), 912-916.

Khandker, R.K., Dulski, J.D., Kilpatrick, J.B., Ellis, R.P., Mitchell, J.B. \& Baine, W.B. (2000). A decision model and cost-effectiveness analysis of colorectal cancer screening and surveillance guidelines for average-risk adults. Int J Techno Assess Health Care, 16, 799-810.

Klabunde, C.N., Schenck, A.P., \& Davis, W.W (2006). Barriers to colorectal cancer screening among medicare consumers. Am J Prev Med, 30(4), 313-9.

Klabunde, C.N., Vernon, S.W., Nadel, M.R., Breen, N., Seeff, L.C., Brown, M.L. (2005). Barriers to colorectal cancer screening: a comparison of reports from primary care physicians and average-risk adults. Med Care, 43(9), 939-44.

Kune, G.A. (2000). Colorectal cancer chemoprevention: aspirin, other NSAIDS, and COX-2 inhibitors. Aust N Z J Surg, 70, 452-455.

Ladabaum, U., Chopra, C.L., Huang, M.D., Scheiman, J.M., Chernew, M.E. \& Fendrick, A.M. (2001). Aspirin as an adjunct to screening for prevention of sporadic colorectal cancer. Annals of Internal Med, 135, 769-783.

Ladabaum, U., Scheiman, J. \& Fendrick, M. (2003). Potential effect of cyclooxygenase-2-specific inhibitors on the prevention of colorectal cancer: a cost-effectiveness analysis. Am J Med, 114(7), 546-554.

Levin, B., Smith, R.A., Feldman, G.E., Colditz, G.A., Fletcher, R.H., Nadel, M., et.al. (2002). Promoting early detection tests for colorectal carcinoma and adenomatous polyps. Cancer, 95(8), 1618-1628.

Lieberman, D.A. \& Weiss, D.G. (2001). Veterans affairs cooperative study group 380. One-time screening for colorectal cancer with combined fecal occult-blood testing and examination of the distal colon. $N$ Engl J Med, 345, 555-560.

Lieberman, D.A., Holub J., Eisen G., Kraemer D. \& Morris C.D. (2005). Utilization of colonoscopy in the United States: results from a national consortium. Gastrointest Endosc, 62(6), 875-83.

Logan, R.F., Little, J., Hawtin, P.G. \& Hardcastle, J.D. (1993). Effect of aspirin and nonsteroidal anti-inflammatory drugs on colorectal adenomas; case-control study of subjects participating in the Nottingham faecal occult blood screening programme. British Medical Journal, 307, 285-289.

Mande,l J.S., Bond, J.H., Church, T.R., Snover, D.C., Bradley, G.M., Schuman, L.M., et. al. (2003). Reducing Mortality from Colorectal Cancer by Screening for Fecal 
Occult Blood. New Eng J Med, 328, 1365-1371.

Mulhall, B.P., Veerappan, G.R., \& Jackson, J.L. (2005). Meta-analysis: computed tomograpic colonography. Ann Intern Med, 142(8), 669-70.

Muscat, J.E., Stellman, S.D., Wynder, E.L. (1994). Nonsteroidal Antiinflammatory Drugs and Colorectal Cancer. Cancer, 74,1847-54.

National Cancer Institute. Conquering colorectal cancer: a blueprint for the future The report of the Colorectal Cancer Progress Review Group. Bethesda, MD: National Cancer Institute, 2000.

National Colorectal Cancer Roundtable (CDC). (2002). Atlanta , Ga.

Nelson, D.B., McQuaid, K.R., Bond, J.H., Leiberman, D.A., Weiss, D.G., Johnston, T.K., (2002). Procedural success and complications of large-scale screening colonoscopy. Gastroinest Endosc, 55, 307-14.

Ness, R.M., Holmes, A.M., Klein, R., Dittus, R. (1999). Utility valuations for outcome states of colorectal cancer. Am J Gastroenterol, 94(6), 1650-7.

Ness, R.M., Holmes, A.M., Klein, R., Dittus, R. (1998). Outcome states of colorectal cancer: identification and description using patient focus groups. Am J Gastroenterol, 93(9), 1491-7.

Ness, R.M., Holmes, A.M., Klein, R., Dittus, R. (2000). Cost-utility of One-time Colonoscopic Screening for Colorectal Cancer at Various Ages. Am J Gastroenterol, 95, 1800-11.

O'Leary, B.A., Olynyk, J.K., Neville, A.M., Platell, C.F. (2004). Cost-effectiveness of colorectal cancer screening: comparison of community-based flexible sigmoidoscopy with fecal occult blood testing and colonoscopy. J Gastroenterol Hepatol, 19(1), 38-47.

Osborn, N.K., Ahlquist, D.A. (2005). Stool screening for colorectal cancer: molecular approaches. Gastroenterology, 128, 192-206.

Pabby A., Suneja, A., Heeren, T., Farraye, F.A. (2005). Flexible sigmoidoscopy for colorectal cancer screening in the elderly. Dig Dis Sci, 50(11), 2147-52.

Peleg, I.I., Maibach, H.T., Brown, S.H., Wilcox, C.M.. (1996). Aspirin and Nonsteroidal Anti-inflammatory Drug Use and the Risk of Subsequent Colorectal Cancer. Arch Intern Med, 25, 773-91.

Peterson, S.K., Vernon, S.W. (2000). A review of patient and physician adherence to colorectal cancer screening guidelines. Semin Colon Rectal Surg, 11, 58-72. 
Pickhardt, P.J., Choi, J.R., Hwang, I., Butler, J.A., Puckett, M.L., Hildebrandt, H.A., et al. (2003). Computed Tomographic Virtual Colonoscopy to Screen for Colorectal Neoplasia in Asymptomatic Adults. New Eng J Med, 349(23), 2191-200.

Pignone, M., Saha, S., Hoerger, T., Mandelblatt, J. (2002). Cost-effectiveness analyses of colorectal cancer screening: a systematic review for the U.S. Preventive services task force. Ann Intern Med, 137, 96-104.

Pineau, B., Paskett, E., Chen, G.J., Espeland, M.A., Phillips, K., Han, J.P., et al. (2003). Virtual Colonoscopy Using Oral Contrast Compared with Colonoscopy for the Detection of Patients with Colorectal Polyps. Gastroenterology, 125(2), 304-10.

Ransohoff, D.F., Land, C.A. and Kuo, H.S. (1991). Colonoscopic Surveillance After Polypectomy: Considerations of Cost Effectiveness. Ann Intern Med, 114(3), 177-82.

Reddy, B., Hirose, Y., Lubet, R., Steele, V., Kelloff, G., Paulson, S., et. al. (2000). Chemoprevention of Colon Cancer by Specific Cyclooxygenase-2 Inhibitor, Celecoxib, Administered during Different Stages of Carcinogenesis. Cancer, 60, 293297.

Reeves, M.J., Newcomb, P.A., Trentham-Dietz, A., Storer, B.E., Remington, P.L. (1996). Nonsteroidal Anti-inflammatory Drug Use and Protection Against Colorectal Cancer in Women. Cancer Epidemiol Biomarkers Prev, 5, 955-60.

Robertson, D., Greenbert, E., Beach, M, Sandler, R., Ahnen, D., Haile, R., el. al. (2005). Colorectal cancer in patients under close colonoscopic surveillance. Gastroenterology, 129, 34-41.

Sandler, R.S., Halabi, S., Baron, J.A., et. al. (2003). A Randomized Trial of Aspirin to Prevent Colorectal Adenomas in Patients with Previous Colorectal Cancer, 2003. New Eng J Med, 348, 883-890.

Schrag, D., Weeks, J. (1999). Costs and cost-effectiveness of colorectal cancer prevention and therapy. Semin Onco, 26(5), 561-568.

Selby, J.V., Friedman, G.D., Quesenberry, C.P., Weiss, N.S. (1992). A case-control study of screening sigmoidoscopy and mortality from colorectal cancer. $N$ Engl $J$ Med, 326(10), 653-7.

Solomon, S.D., McMurray, J.J., Pfeffer, M.A., Wittes, J., Fowler, R., Finn, P. et al. (2005). Cardiovascular risk associated with celecoxib in a clinical trial for colorectal adenoma prevention. N Engl J Med, 352(11), 1071-80.

Sonnenberg, A., Delco, F., Bauefreind, P. (1999). Is Colonoscopy a Cost-Effective Option to Screen for Colorectal Cancer? Am J Gastroenterol, 94(8), 2268-74. 
Sonnenberg, A., Delco, F., Inadomi, J.M. (2000). Cost-effectiveness of Colonoscopy in Screening for Colorectal Cancer. Ann Intern Med, 133, 573-84.

Steinbach, G., Lynch, P.M., Phillips, R.K., Wallace, M.H., Hawk, E., Gordon, G.B., et al. (2000). The effect of celecoxib, a cyclooxygenase-2 inhibitor, in familial adenomatous polyposis. $N$ Engl J Med, 342(26), 1946-52.

Suh, O., Mettlin, C., Petrelli, N. (1993). Aspirin Use, Cancer, and Polyps of the Large Bowel. Cancer, 72, 1171-7.

Surveillance, Epidemiology, and End Results (SEER) Program, National Cancer Institute, DCCPS, Surveillance Research Program, Cancer Statistics Branch, release April 2004, based on the November 2003 submission.

Tabbarah, M., Nowalk, M.P., Raymund, M., Jewell, I.K., Zimmerman, R.K. (2005). Barriers and facilitators of colon cancer screening among patients at faith-based neighborhood health centers. J Comm Health, 30(1), 55-74.

Taplin, S.H., Barlow, W., Urban, N., Mandelson, M.T., Timlin, D.J., Ichikawa, L., et al. (1995). Stage, age, comorbidity, and direct costs of colon, prostate, and breast cancer care. J Natl Cancer Inst, 87, 417-26.

Thun, M.J., Namboodiri, M.M., Calle, E.E., Flanders, W.D., Heath, C.W. Jr. (1993). Aspirin Use and Risk of Fatal Cancer. Cancer Research, 53, 1322-7.

Thun, M.J., Namboodiri, M.M., Heath, C.W. Jr. (1991). Aspirin Use and Reduced Risk of Fatal Colon Cancer. N Engl J Med, 325, 1593-6.

Trentham-Dietz, A., Remington, P.L., Moinpour, C.M., Hampton, J.M., Sapp, A.L., Newcomb, P.A. (2003). Health-related quality of life in female long-term colorectal cancer survivors. Oncologist, 8(4), 342-9.

Tuynman, J.B., Hulscher, J.B., Steller, E.P., van Lanschot, J.J., Richel, D.J. (2003). Cyclooxygenase(COX)-2-inhibition in the prevention and treatment of colorectal carcinoma. Ned Tijdschr Geneeskd, 147(45), 2207-12.

U.S. Preventive Services Task Force. Guide to Clinical Preventive Services. $2^{\text {nd }}$ ed. Baltimore: Williams \& Wilkins. 1996:89-103.

U.S. Preventive Services Task Force. Screening for colorectal cancer: recommendations and rationale. Ann Intern Med 2002 Jul 16;137(2):129-31.

Vernon, S.W. (1997). Participation in colorectal cancer screening: a review. J Natl Cancer Inst, 89, 1406-1422. 
Vijan, S., Hwang, E.W., Hofer, T.P., Hayward, R.A. (2001). Which Colon Cancer Screening Test? A Comparison of Costs, Effectiveness, and Compliance. Am J Med, 111, 593-601.

Wagner, J., Tunis, S., Brown, M., Ching, A., Almeida, R. (1996). Cost-effectiveness of Colorectal Cancer Screening in Average-Risk Adults. In: Young G, Rozen P, Leven B, eds. London: Prevention and Early Detection of Colorectal Cancer.

Suanders, 321-56.

Wagner, J. (1990). From the congressional office of technology and assessment. JAMA, 264(21), 2732.

Winawer, S., Fletcher, R., Rex, D., Bond, J., Burt, R., Ferrucci, J., et al. (2003). Colorectal Cancer Screening and Surveillance: Clinical Guidelines and Rationale Update Based on New Evidence. Gastroenterology, 124, 544-560.

Woolf, S. (2000). Overcoming the Barriers to Change: Screening for Colorectal Cancer. Am Fam Physician, 61, 1621-2,1628

World Health Organization, Department of Mental Health. (1998). Annotated bibliography of the WHO quality of life assessment instrument- WHOQOL. 